\title{
Radial and tangential migration of telencephalic somatostatin neurons originated from the mouse diagonal area
}

\author{
Luis Puelles $^{1,5} \cdot$ N. Morales-Delgado ${ }^{1} \cdot$ P. Merchán ${ }^{1,3} \cdot$ B. Castro-Robles ${ }^{2,4}$ • \\ M. Martínez-de-la-Torre ${ }^{1} \cdot$ C. Díaz ${ }^{2} \cdot$ J. L. Ferran ${ }^{1}$
}

Received: 7 April 2015/Accepted: 7 July 2015/Published online: 19 July 2015

(c) The Author(s) 2015. This article is published with open access at Springerlink.com

\begin{abstract}
The telencephalic subpallium is the source of various GABAergic interneuron cohorts that invade the pallium via tangential migration. Based on genoarchitectonic studies, the subpallium has been subdivided into four major domains: striatum, pallidum, diagonal area and preoptic area (Puelles et al. 2013; Allen Developing Mouse Brain Atlas), and a larger set of molecularly distinct progenitor areas (Flames et al. 2007). Fate mapping, genetic lineage-tracing studies, and other approaches have suggested that each subpallial subdivision produces specific sorts of inhibitory interneurons, distinguished by differential peptidic content, which are distributed tangentially to
\end{abstract}

L. Puelles, N. Morales-Delgado and P. Merchán contributed equally to this work.

Electronic supplementary material The online version of this article (doi:10.1007/s00429-015-1086-8) contains supplementary material, which is available to authorized users.

Luis Puelles

puelles@um.es

1 Department of Human Anatomy and Psychobiology, School of Medicine, University of Murcia, Murcia E30071, Spain

2 Department of Medical Sciences, School of Medicine, University of Castilla-La Mancha, Albacete E02006, Spain

3 Present Address: Division of Developmental Biology, Perinatal Institute, Cincinnati Children's Hospital Medical Center, University of Cincinnati College of Medicine, Cincinnati, OH 45229, USA

4 Present Address: Department of Experimental Medicine, Laboratory of Brain Development and Evolution, Institute of Biomedical Research of Lleida, University of Lleida, Lleida, Spain

5 Department of Human Anatomy and Psychobiology, Faculty of Medicine, University of Murcia, 30100 Murcia, Spain pallial and subpallial target territories (e.g., olfactory bulb, isocortex, hippocampus, pallial and subpallial amygdala, striatum, pallidum, septum). In this report, we map descriptively the early differentiation and apparent migratory dispersion of mouse subpallial somatostatin-expressing (Sst) cells from E10.5 onward, comparing their topography with the expression patterns of the genes $D l x 5$, Gbx2, Lhx7-8, Nkx2.1, Nkx5.1 (Hmx3), and Shh, which variously label parts of the subpallium. Whereas some experimental results suggest that $S s t$ cells are pallidal, our data reveal that many, if not most, telencephalic Sst cells derive from de diagonal area $(\mathrm{Dg})$. Sst-positive cells initially only present at the embryonic Dg selectively populate radially the medial part of the bed nucleus striae terminalis (from paraseptal to amygdaloid regions) and part of the central amygdala; they also invade tangentially the striatum, while eschewing the globus pallidum and the preoptic area, and integrate within most cortical and nuclear pallial areas between E10.5 and E16.5.

Keywords Forebrain interneurons - Secondary prosencephalon $\cdot$ Subpallium $\cdot$ Pallidum $\cdot$ Medial ganglionic eminence $\cdot$ Preoptic area $\cdot$ Entopeduncular area $\cdot$ Striatum · Cortex

$\begin{array}{ll}\text { Abbreviations } \\ \text { A } & \text { Amygdala } \\ \text { AA } & \text { Anterior amygdala } \\ \text { ac } & \text { Anterior commissure } \\ \text { Acb } & \text { Accumbens nucleus } \\ \text { AH } & \text { Anterior lobe of hypophysis } \\ \text { AHi } & \text { Amygdalo-hippocampal area } \\ \text { Amygd } & \text { Amygdala } \\ \text { AOA } & \text { Anterior olfactory area } \\ \text { APi } & \text { Amygdaloid piriform area }\end{array}$




\begin{tabular}{|c|c|c|c|}
\hline B & Basal magnocellular nucleus of Meynert & MePV & Medial posteroventral nucleus of the amygdala \\
\hline $\mathrm{BEC}$ & Bed nucleus of external capsule & MGE & Medial ganglionic eminence \\
\hline BL & Basolateral amygdaloid nucleus & NCX & Neocortex \\
\hline $\mathrm{BM}$ & Basomedial amygdaloid nucleus & NLOT & Nucleus of the lateral olfactory tract \\
\hline bp & Basal plate & NLOTm & Migration stream of the nucleus of the lateral \\
\hline BST & Bed nucleus of the stria terminalis & & olfactory tract \\
\hline BSTa & Amygdaloid BST nucleus & OA & Olfactory amygdala \\
\hline BSTL & Lateral (pallidal) part of BST & OB & Olfactory bulb \\
\hline BSTLsc & Supracapsular part of BSTL & OCx & Orbitary cortex \\
\hline BSTM & Medial (diagonal) part of BST & os & Optic stalk \\
\hline BSTMa & Anterior part of BSTM & ot & Optic tract \\
\hline BSTMsc & Supracapsular part of BSTM & OT & Olfactory tuberculum \\
\hline BSTMps & Paraseptal part of BSTM & OtpMS & Otp-positive cells migratory stream \\
\hline $\mathrm{C}$ & Central region of the subpallium & p2 & Prosomere 2 \\
\hline $\mathrm{Ce}$ & Central amygdaloid nucleus & p3 & Prosomere 3 \\
\hline $\mathrm{CeC}$ & Capsular part of $\mathrm{Ce}$ & $\mathrm{Pa}$ & Paraventricular hypothalamic area \\
\hline $\mathrm{CeL}$ & Lateral part of $\mathrm{Ce}$ & Pal & Pallidum \\
\hline $\mathrm{CeM}$ & Medial part of $\mathrm{Ce}$ & PalSe & Pallidal septum \\
\hline CGE & Caudal ganglionic eminence & PallA & Pallial amygdala \\
\hline $\mathrm{CL}$ & Claustrum & ped & Peduncle \\
\hline $\mathrm{CL} / \mathrm{I}$ & Claustro-insular complex & PHy & Peduncular hypothalamus \\
\hline $\mathrm{CP}$ & Cortical plate & PirCx & Piriform cortex \\
\hline $\mathrm{CPu}$ & Caudate putamen & PirCx III & Layer III of PirCx \\
\hline $\mathrm{Cx}$ & Cortex & PLCo & Posterolateral cortical amygdaloid nucleus \\
\hline DB & Diagonal band nucleus & PMCo & Posteromedial cortical amygdaloid nucleus \\
\hline $\mathrm{db}$ & Diagonal band & POA & Preoptic area \\
\hline $\operatorname{Dg}$ & Subpallial diagonal domain & POA1 & Area 1 of POA \\
\hline $\operatorname{DgA}$ & Amygdaloid diagonal area & POA2 & Area 2 of POA \\
\hline $\mathrm{DgC}$ & Central diagonal area & POASe & Preoptic septum \\
\hline DgMC & Magnocellular diagonal nucleus & $\mathrm{POH}$ & Preopto-hypothalamic transition area \\
\hline $\mathrm{DgSe}$ & Diagonal septum & PT & Pretectum \\
\hline EGP & Globus pallidus, external segment & PSe & Paraseptal subpallium \\
\hline $\mathrm{ERh}$ & Entorhinal cortex & PTh & Prethalamus \\
\hline $\mathrm{FCx}$ & Frontal cortex & PThE & Prethalamic eminence \\
\hline GP & Globus pallidus & $\mathrm{Rh}$ & Rhombencephalon \\
\hline $\mathrm{Hb}$ & Habenula & $\mathrm{S}$ & Subiculum \\
\hline HDB & Diagonal band, horizontal nucleus & $\mathrm{Se}$ & Septum \\
\hline $\mathrm{Hi}$ & Hippocampus & $\mathrm{SeDg}$ & Septo-diagonal area (paraseptal diagonal \\
\hline hp1 & Hypothalamic prosomere 1 & & domain) \\
\hline hp2 & Hypothalamic prosomere 2 & SePal & Septo-pallidal area (paraseptal pallidal \\
\hline Hy & Hypothalamus & & domain) \\
\hline ic & Internal capsule & SI & Substantia innominata \\
\hline $\mathrm{ICx}$ & Insular cortex & SSpM & Superficial subpallial migration stream \\
\hline ILCx & Infralimbic cortex & slt & Sulcus terminalis \\
\hline IGP & Globus pallidus, internal segment & st & Stria terminalis \\
\hline IPAC & Intersticial nucleus of the posterior limb of & St & Striatum \\
\hline & the anterior commisure & StSe & Striatal septum \\
\hline $\mathrm{L}$ & Lateral amygdalar nucleus & Subpial & Subpial migratory pathway \\
\hline LGE & Lateral ganglionic eminence & migr & \\
\hline lot & Lateral olfactory tract & Subvent & Subventricular migratory pathway \\
\hline lt & Lamina terminalis & migr & \\
\hline $\operatorname{lv}$ & Lateral ventricle & SvSpM & Subventricular subpallial migratory stream \\
\hline MA & Medial amygdala & Tel & Telencephalon \\
\hline
\end{tabular}


Th Thalamus

THy Terminal hypothalamus

VPal Ventral pallidum

VSt Ventral striatum

vZ Ventricular zone

Zli Zona limitans interthalamica

\section{Introduction}

The identity of neuron types produced within a specific brain region is the result of progressive patterning and consequent fate specification of progenitors during early ontogeny. Establishment of a unique molecular profile at a given progenitor domain allows it to generate a particular neuronal cell type (or several of them, either sequentially, or in a salt-and-pepper pattern). Such neurons are presumed to be different at least in some subtle aspects from those produced in adjoining areas, irrespective that some part of the respective molecular profiles may be shared. Some neuronal derivatives aggregate radially within the local mantle zone, whereas others may migrate tangentially into neighbouring or distant brain areas. The latter is a wellknown phenomenon in the telencephalon, where various subpallial cell populations migrate into other parts of the subpallium or into the pallium, contributing diverse contingents of inhibitory interneurons to local circuitry (reviewed in Marín and Rubenstein 2003; Gelman et al. 2009, 2012; Marin 2013). In this report, we examine areally restricted subpallial production, and subsequent migratory dispersion, of telencephalic somatostatin (Sst) neurons into various subpallial and pallial target domains, highlighting their participation in the radial development of the medial bed nucleus striae terminalis, and the lateral part of the central amygdalar nucleus (areas whose development was hitherto largely obscure).

We presently understand the subpallium as consisting of four main partitions stretched along the septoamygdaloid axis; these are, from medial to lateral: preoptic area $(\mathrm{POA})$, diagonal area $(\mathrm{Dg})$, pallidum $(\mathrm{Pal})$, and striatum (St) (Fig. 1a, b; Allen Developing Mouse Brain Atlas; Medina and Abellan 2012; Puelles et al. 2013). Historically, the Dg was first identified as anterior entopeduncular area (AEP; Bulfone et al, 1993; Puelles and Rubenstein, 1993; Rubenstein et al. 1994). This is a somewhat misleading and unsatisfactory term, since it refers exclusively to an intrapeduncular locus and not to a full histogenetic domain. Therefore, it was later substituted by some authors by rough topographic reference to the part of the MGE (caudoventral, caudomedial or ventral) occupied by this domain—cvMGE/cmMGE/vMGE; separately, Flames et al. (2007) identified the corresponding progenitor domain as pMGE5 (see Fig. 1c). Finding all these names anatomically imprecise and not distinctive enough, Puelles proposed the diagonal area (Dg) name while working on the terminology used in the Allen Developing Mouse Brain Atlas (developingmouse.brain-map.org; online since 2009; Puelles et al, 2013). This name refers to the inclusion within the referred histogenetic domain of the classical diagonal band nuclei and the related substantia innominata. These landmarks allow easy anatomic identification of the Dg with regard to Pal and POA. A comparable set of four areal subdivisions (septal, paraseptal, central, and amygdaloid) can be distinguished generically across each of these main domains, forming parallel series along the septoamygdaloid axis (Fig. 1b). These subareas were systematized in the Allen Developing Mouse Brain Atlas (http://www.developingmouse.brain-map.org), as well as by Puelles et al. (2013). The central parts of POA, Dg, and Pal participate in the MGE, whereas the central St occupies most of the LGE (Fig. 1b; Flames et al. 2007). The corresponding paraseptal parts (e.g., nucleus accumbens) are found rostromedially, at the locus where the POA, Dg, Pal, and St areas extend under the lateral ventricle and the interventricular foramen into the medial septal wall; septal subdivisions corresponding to the POA, Dg, Pal, and St domains can be identified as well (Fig. 1b; Puelles et al. 2000, 2004; Flames et al. 2007). At the opposite end of the septoamygdaloid axis, amygdaloid parts of St, Pall, and Dg conform the CGE, which is also medially continuous with the preopto-hypothalamic transition area; the latter may be added to the extended amygdala concept (POH; Fig. 1b).

The molecular phenotype of the subpallial ventricular and subventricular zone was used by Flames et al. (2007) to define 18 molecularly distinct progenitor areas, mapped to the septum, ganglionic eminences, and preoptic area; part of these areas are represented in our Fig. 1c (pLGE1-4, not shown; pMGE1-5; pPOA1,2, pPOH, pSe1-6).

Most neurons generated from these different subpallial progenitor areas are GABAergic. Some of them settle radially into the local mantle and differentiate as projection neurons or interneurons. In addition, groups of GABAergic and cholinergic neurons migrate tangentially from some subpallial areas into other subpallial areas (Marín and Rubenstein 2003; Gelman et al. 2009, 2012; Marin 2013). Other GABAergic interneurons of various subpallial origins migrate into the pallium (both cortex and nuclei), constituting in the adult roughly $20 \%$ of the total cortical population. The elements that migrate into the cortex have been classified into three or four non-overlapping populations expressing either parvalbumin (PV), somatostatin (SST), or calretinin/vasointestinal peptide (CR/VIP), with the possible addition of neuropeptide Y (NPY/reelin) cells (Marin and Rubenstein 2001, 2003; Wonders and Anderson 2006; Gelman and Marin 2010; Miyoshi et al. 2010; Xu et al. 2010; Lee et al. 2010). 

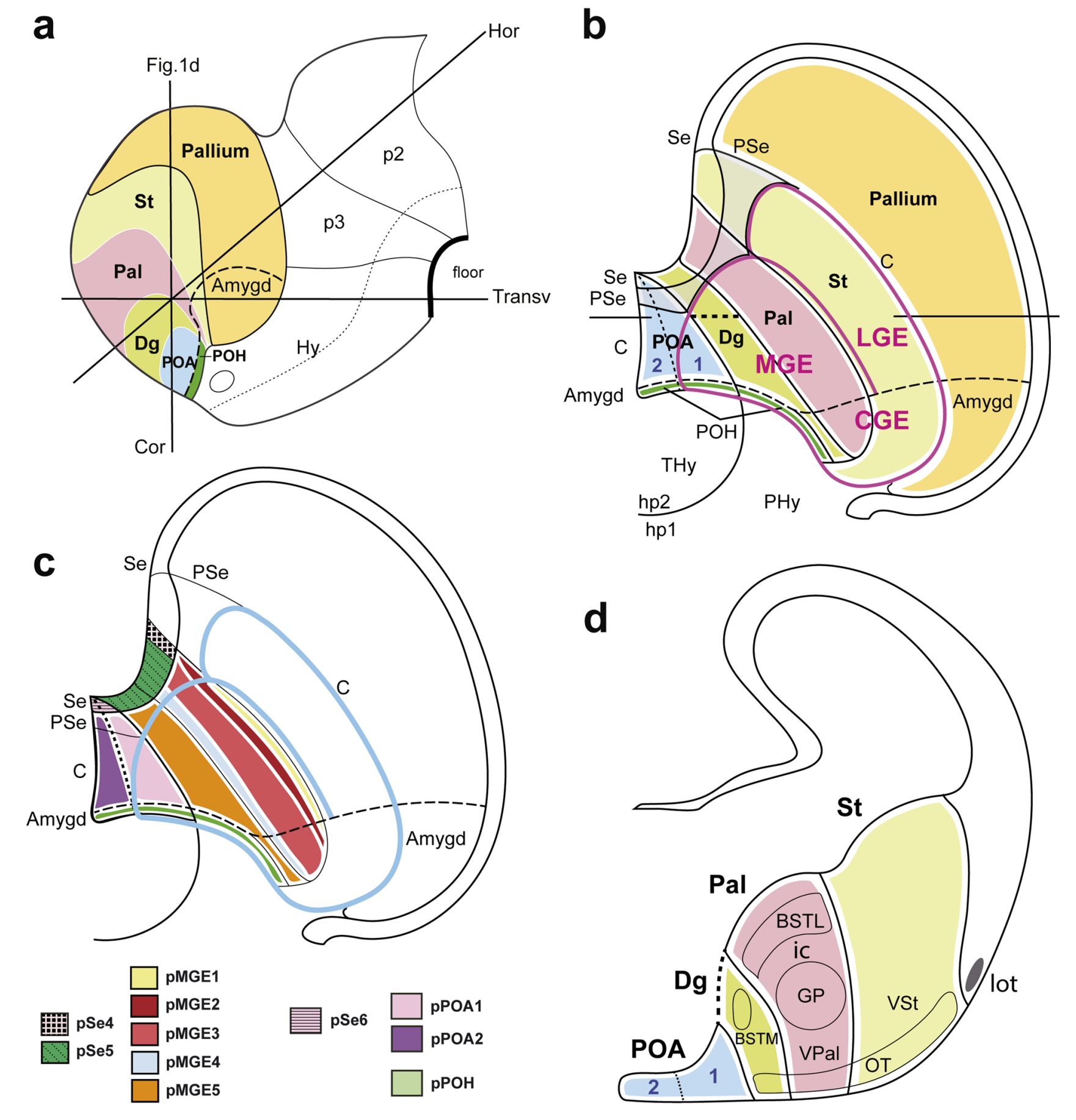

\section{Somatostatin neurons in the telencephalon}

The hormone/neuropeptide somatostatin (SST; also known as somatotropin-release inhibiting factor) was first isolated from hypothalamic extracts on the basis of its ability to inhibit growth hormone secretion from the anterior pituitary (Brazeau et al. 1973). The somatostatin mRNA

precursor is translated to produce a large inactive pre-prosomatostatin peptide (116 amino acids; PPSST); its posttranslational enzymatic cleavage yields two biologically active products, somatostin 14 (14 amino acids; SST-14) and somatostin 28 (28 amino acids; SST-28), which have neurotransmitter and neuromodulator roles (Kumar and Grant 2010). SST is known to be involved in granule cell 
4Fig. 1 - Schemas illustrating the relative topography of subpallial subdivisions. a Schema of a left-side view of the embryonic brain, indicating in colors the telencephalic region. The pallium (orange) is separated from the subpallium by a black line. The subpallium appears divided into four domains: striatum (yellow St), pallidum (pink $\mathrm{Pal})$, diagonal area (green $\mathrm{Dg}$ ), and preoptic area (blue POA). The preopto-hypothalamic area (dark green $\mathrm{POH}$ ), a part of POA, abbuts the telencephalic/preoptic border with respect to the terminal and peduncular parts of the hypothalamus (THy, $\mathrm{PHy}$ ); The $\mathrm{POH}$ is continuous laterally across the hemispheric stalk with the subpallial and pallial amygdala (all three enclosed by the dash line). The longitudinal alar/basal boundary of the forebrain is depicted as a dotted line; the forebrain floor plate is marked by a thick black line; p2-p3 refer to diencephalic prosomeres. The coronal (Cor), horizontal (Hor), and transverse (Transv) planes of section used in this study are indicated; note the Hor and Transv planes are oriented relative to the prosomeric length axis and floor plate, while the Cor plane is arbitrary, and corresponds to the coronal section schema in (d). b Two-dimensional schema looking down on a flattened view of the right hemisphere, in which the four subpallial domains are mapped relative to the septal region, the hypothalamus, the pallium, and the medial, lateral and caudal ganglionic eminences (red contour; MGE, LGE, CGE; note the mixed composition of the MGE). The color code, areal names, and dash line used in (a) apply here to the 'central' or principal region of the subpallium in (b). The septo-amygdaloid axis can be imagined, with an obliquity that characterizes particularly the evaginated subpallial domains ( $\mathrm{St}$, Pal; less so $\mathrm{Dg}$, or POA). The septal end of the subpallium (Se) is strictly septal, and contains in principle the topological dorsal end of all four subpallial domains (note the commissural septal midline lies at the telencephalic roof plate). Intercalated between the septal (Se) and the central subpallial sectors (C) there appears the paraseptal subpallial sector (PSe), which connects them (passing under the interventricular foramen), whereas the subpallial amygdala found within the CGE represents the amygdaloid subpallial sector (Amygd). The four main subpallial domains thus stretch from the septum into the amygdala. The Dg domain, of particular interest in the present study, lies precisely at the hemispheric stalk. The level of the schematic coronal section shown in (d) is indicated. c This schema is basically a reproduction of (b), used for tentative mapping of the Se, MGE, and POA progenitor domains distinguished by Flames et al. (2007) relative to the ganglionic eminences (blue contour line); the color code indicated for these areas is slightly modified (for visibility) from that used by these authors; note the LGE progenitor areas are not represented (not needed in the present context). d Conventional schema of a coronal cross section through the telencephalon, in which the central subpallial domains are intersected side by side-see section plane in (a) and (b). Comparison of the dashed ventricular contour of the sectioned Dg (pMGE5) area with the dash line in (b) illustrates our idea that, in three dimensions, this domain is not a localized neuroepithelial patch, but an obliquely elongated band. POA and Dg converge rostrodorsally at the crossing of the anterior commissure (septocommissural preoptic area)

migration during cerebellar development (Epelbaum et al. 1994; Yacubova and Komuro 2002; Le Verche et al. 2009). Several mapping studies performed in embryonic, postnatal, and adult mice showed that SST has a wide central nervous system distribution that includes cerebral cortex, hippocampus, striatum, amygdala, olfactory system, hypothalamus, diencephalon, midbrain, and brainstem (Roberts et al. 1982, Moga and Gray 1985, Gray and
Magnuson 1992; Garcia-Lopez et al. 2008; Viollet et al. 2008; Real et al. 2009; Bupesh et al. 2011a, b; MoralesDelgado et al. 2011).

According to in vitro and in vivo fate-mapping studies, the medial ganglionic eminence (MGE), which is the sum of the central parts of Pal, Dg and POA (Fig. 1b), is currently conceived as the main source of PV+ and SST+ cortical interneurons (Xu et al. 2004; Butt et al. 2005; Fogarty et al. 2007; Ghanem et al. 2007). However, there is so far no consensus on the specific origin of SST neurons within the MGE. The 'dorsal' MGE area (which includes mainly the pMGE1 subdomain of Flames et al. 2007, which differentially expresses $N k x 6.2$ ) was specifically proposed as the main source of Sst-expressing cortical interneurons by Wonders et al. (2008), a conclusion that was supported by other genetic lineage tracings done in mice (e.g., Sousa et al. 2009). However, lineage studies using $N k x 2.1 C r e$ labeling suggested that most SST + cells derive from the 'central and ventral MGE' subregion, which corresponds roughly to the pMGE5 area of Flames et al. 2007 (Fogarty et al. 2007; Xu et al. 2008). An area that seems likewise to correspond to pMGE5, but that was referred to as 'caudal and medial MGE', was reported to be a source of calbindin-containing neurons that enter the pallial amygdala (Neri et al. 2002; Legaz et al. 2005). Several reports of Medina and collaborators concluded that the 'caudoventral MGE' (presumably still the same area), contributes SST+ interneurons to the amygdala (GarcíaLópez et al. 2008; Bupesh et al. 2011a, b; Medina and Abellan 2012; see also Real et al. 2009). We hold that these positional terms (caudoventral/caudomedial MGE) all essentially refer to the Dg domain of our terminology, which we conceive as elongated along the septoamygdaloid axis (Fig. 1b), whereas the cited authors seem to think of a more circumscribed neuroepithelial patch. Most of the cited studies using transgenic mice analyzed their data from E12.5 or E13.5 onward, whereas the earliest subpallial Sst cells appear at E10.5 (present results). The present full descriptive data accordingly provide information about the appropriateness of E12.5/E13.5 material for the deductions obtained from those transgenic experiments.

In the present work, we used the updated model of subpallial areal subdivisions (Fig. 1) to analyze in detail the spatiotemporal distribution of Sst mRNA expression during early development in the mouse telencephalon, aiming to trace overall developmental distribution of this cell type in the telencephalon, starting at initial stages. In order to illuminate the issue of a potential localized source within a subdomain of the MGE (the dorsal Pal, or pMGE1, and the Dg, or pMGE5, as suggested alternative candidates), Sst mRNA expression was compared in adjacent sections with $S h h$ signal and several differentially expressed transcription factors (Dlx5, Gbx2, Lhx7-8, 
Nkx2.1, Nkx5.1) over the embryonic period E9.5 to E16.5. Our study led us to pinpoint the Dg domain as the first subpallial domain that contains cells expressing $S s t$ in its mantle (from E10.5 onwards). At this time point, these diagonal $S s t$ cells clearly are topographically distinct from pallidal Gbx2-positive cells, as well as from preoptic $S h h /$ Nkx5.1-positive derivatives. At E12.5, many Sst cells apparently derived from the $\mathrm{Dg}$ domain have already invaded tangentially the striatal mantle (traversing subpially the pallidal domain, but clearly eschewing its central mantle) and start to migrate subpially past the LGE into the pallium. The tangentially migrated Sst population increases markedly subsequently, but no other locus (in dorsal Pal or elsewhere) was found outside the Dg where Sst cells clearly seem to arise from the ventricular or subventricular zone. However, our material may not be sufficient to negate altogether that possibility, since the expression of Sst may start after some delay. Our analysis accordingly suggests that numerous Sst-expressing neurons that colonize cortical and subcortical structures seem to derive tangentially from the Dg domain. Our data indicate that the Sst-positive components that eventually populate the medial bed nucleus striae terminalis complex and a part of the central amygdala represent radial derivatives of the $\mathrm{Dg}$, produced along the length of its septoamygdaloid dimension.

\section{Materials and methods}

All experimental procedures involving use and care of laboratory animals were conducted in compliance with the current normative standards of the European Community (86/609/EEC) and the Spanish Government (Royal Decree, 1201/2005; Law 32/2007).

\section{Animals and tissue preparation}

For the present research, Swiss albino mouse embryos were collected from embryonic day (E) 9.5-16.5 after fertilization (adult specimens were collected as well). Noon on the day of the appearance of the vaginal plug was considered day 0.5 of gestation (E0.5). Mouse embryos were separately staged according to the Theiler stages (TS; Theiler 1989). For every embryonic age, we examined three to five mouse embryos. Timed-pregnant dams were sacrificed by cervical dislocation and embryos were immediately removed by cesarean section, anesthetized by cold, and decapitated. The heads were immersion-fixed in freshly made $4 \%$ paraformaldehyde in $0.1 \mathrm{M}$ phosphate-buffered saline (PBS, pH 7.4). The adult specimens were perfused under anesthesia with the same solution. The brains were then dissected out and post-fixed overnight at $4{ }^{\circ} \mathrm{C}$ in the same fixative. For cryostat sections, embryonic brains were first transferred to $30 \%$ sucrose in $0.1 \mathrm{M}$ PBS for $24 \mathrm{~h}$ at $4{ }^{\circ} \mathrm{C}$, and then placed in $15 \%$ gelatin $/ 20 \%$ sucrose solution at $37{ }^{\circ} \mathrm{C}$ until they sank. They were next embedded in the same solution, hardening the blocks at $4{ }^{\circ} \mathrm{C}$. These primary blocks were subsequently trimmed in order to establish the desired sectioning plane, and were then frozen for $2 \mathrm{~min}$ in isopentane cooled to $-55^{\circ} \mathrm{C}$ in dry ice, and either kept frozen for future use, or placed in proper orientation upon the cryostat chuck. Sections were obtained serially $16-20 \mu \mathrm{m}$-thick in either the sagittal or transverse planes through the secondary prosencephalon on a Leica CM3500 S cryostat, and mounted as 3-4 parallel series onto Superfrost-plus slides (Menzel-Gläser, Braunschweig, Germany). These were stored at $-20{ }^{\circ} \mathrm{C}$ until they were processed for in situ hybridization or immunohistochemistry. Some brains, including the adult ones, were embedded in $4 \%$ low-melting point agarose (Pronadisa, Torrejón de Ardoz, Madrid, Spain, Cat. 8008), cut on a Leica VT1000 S vibratome $90 \mu \mathrm{m}$-thick in the sagittal or coronal planes, and processed as free-floating sections (Ferran et al. 2015a, b).

\section{RT-PCR}

Lhx8 and Nkx5.1 (Hmx3) cDNA fragments were obtained by reverse transcription (RT). RNA was individually extracted with Trizol reagent (Invitrogen, Carlsbad, CA, Cat. 10296-028) from freshly dissected brains of Mus musculus embryos at E10.5, E12.5, and E14.5. The RNA was treated with DNase I (Invitrogen, Cat. 18068-015) for $15 \mathrm{~min}$ at room temperature (RT), and the enzyme was then inactivated at $65{ }^{\circ} \mathrm{C}$. Afterward, RNA samples were converted to single-stranded cDNA with Superscript III reverse transcriptase (Invitrogen, Cat. 18080-044) and oligo-dT-anchored primers. The resulting first-strand cDNA $(0.5 \mu \mathrm{l}$ of the reverse transcription reaction) was used as a template for the PCR reaction, which was performed in presence of Taq polymerase (Promega, Cat. M8305) and the following gene-specific primers for $L h x 8$ and Nkx5.1 (Hmx3) mRNA.

mLhx8F: $5^{\prime}$-AGCTGGTATGTGACGAGCA- $3^{\prime}$

mLhx8R: 5'-AGAATGGTTGGGACTGACG-3'

mNkx5.1F: $5^{\prime}$-GACCACAAGGAGCTGGACTC- $3^{\prime}$

mNkx5.1R: 5'-TAAGAGGAGAAGCGCCTCAA-3'

The PCR conditions used were an initial denaturation step at $94{ }^{\circ} \mathrm{C}$ for $5 \mathrm{~min}$, then 35 cycles $\left[30 \mathrm{~s}\right.$ at $94{ }^{\circ} \mathrm{C}$, plus $1 \mathrm{~min}$ at $\mathrm{Tm}$ temperature $\left(58^{\circ} \mathrm{C}\right)$, and $1 \mathrm{~min}$ at $72^{\circ} \mathrm{C}$ ], followed by $20 \mathrm{~min}$ at $72{ }^{\circ} \mathrm{C}$. The PCR products were cloned into the pGEM-T Easy Vector (Promega, Cat. A1360), and sequenced (SAI, University of Murcia). 


\section{In situ hybridization}

The embryos were processed for in situ hybridization with digoxigenin-UTP-labeled antisense riboprobes. Sense and antisense digoxigenin-labeled riboprobes for mouse Dlx5, Gbx2, Lhx8, Nkx2.1, Nkx5.1, Shh, and Sst were synthetized with a kit, following the manufacteŕs recommendations (Roche Diagnostics S.L. Applied Science, Barcelona, Spain), and applying specific polymerases (Fermentas, Madrid, Spain). Plasmid information is provided in Table 1. In situ hybridization on cryosections was performed basically as described by Ferran et al. (2015a, b). Sections were not treated with proteinase $\mathrm{K}$ before prehybridization. Hybridizations were carried out overnight at $72{ }^{\circ} \mathrm{C}$. Hybridization experiments on floating sections were performed following the protocol described by Ferran et al. (2015b). After hybridization, all sections were washed and incubated in a solution containing alkaline phosphatasecoupled anti-digoxigenin antibody (diluted 1:3.500; Roche Diagnostics). Nitroblue tetrazolium/5-bromo-4-chloro-3indolyl phosphate (NBT/BCIP; Roche) solution was then used as chromogenic substrate for the final alkaline phosphatase reaction (Boehringer, Mannheim, Germany). No specific signal was obtained with sense probes (data not shown). To identify the diverse telencephalic cell masses during forebrain development, we consulted atlases of the developing mouse brain (e.g., Allen Developing Mouse Brain Atlas, http://www.developingmouse.brain-map.org), as well as our own previously published studies on the subject.

\section{Immunohistochemistry}

Our immunohistochemical reaction protocol has been described in detail elsewhere (Bardet et al. 2006; Ferran et al. 2015b). Rabbit polyclonal antiserum against rat NKX2.1 and monoclonal antiserum against rat tyrosine hydroxilase were diluted 1:1000 for use (anti-thyroid transcription factor 1 or TTF-1; Biopat Immunotechnologies, Caserta, Italy; no. PA 0100; anti-TH, Diaserin,
Stillwater, MN, USA). After washes, the sections were incubated with biotinylated goat anti-rabbit or goat antimouse (Vector Laboratories, CA, USA; used at 1:200 dilution) followed by a streptavidin-peroxidase complex (Vectastain-ABC kit; Vector Laboratories; $0.001 \%$ dilution), applied for $1 \mathrm{~h}$ at room temperature. Peroxidase activity was developed with $0.03 \% 3,3^{\prime}$-diaminobenzidine (Sigma; St Louis; MO, USA), plus $0.003 \%$ hydrogen peroxidase. After immunohistochemical and hybridization labeling, the slides were washed several times in PBS, air dried and coverslipped with Cytoseal 60 (Thermo Scientific, Ref. 8310-16) or Mowiol (Calbiochem, Bad Soden, Germany, Ref. 475904). We verified the specificity of the antibodies by performing parallel control experiments that omitted the primary antibody, checking that no residual immunostaining was detected (data not shown).

\section{Imaging}

Whole-slide digital images were acquired with a ScanScope CS digital slide scanner at high resolution (Aperio Technologies, Inc.; Vista, CA, USA). After scanning, the visualization and capture of images of adjacent labeled sections were carried out by using the Aperio software ImageScope. The images were corrected for contrast, focus, and brightness. In order to compare different gene expression patterns, the images of adjacent sections reacted with different markers were superposed and artificially pseudocolored (from blue to red or green) with Photoshop CS3. The plates were labeled using Adobe Photoshop Illustrator CS2 (Adobe Systems Inc., San José, CA, USA).

\section{Results}

\section{Telencephalic Sst mRNA expression in the subpallium starts at E10.5}

The first telencephalic Sst signal was detected in a restricted sector of the subpallial mantle zone from E10.5

Table 1 List of the gene probes used for ISH and their principal characteristics

\begin{tabular}{llclll}
\hline Gene symbol & NCBI accession no. & Size (bp) & Position & Linearization enzyme/polymerase & Publication/Laboratory \\
\hline Dlx5 & NM_010056.2 & 1180 & $106-1285$ & NcoI/Sp6 & Morales-Delgado et al. 2011 \\
Gbx2 & NM_010262.3 & 1040 & $422-1461$ & HindIII/T7 & Martínez S. lab \\
Lhx7-8 & NM_010713.2 & 963 & $176-1138$ & SacII/Sp6 & Present results \\
Nkx2.1 & NM_009385.2 & 2216 & $597-2813$ & SalI/T3 & Rubenstein J.L.R. lab \\
Nkx5.1 & NM_008257.2 & 785 & $487-1271$ & SphI/Sp6 & Present results \\
Otp & NM_011021.2 & 412 & $179-592$ & EcoR1/Sp6 & Morales-Delgado et al. 2011 \\
Shh & NM_009170.2 & 643 & $442-1084$ & HindIII/T3 & McMahon A. lab \\
Sst & NM_009215 & 556 & $6-561$ & NdeI/T7 & Morales-Delgado et al. 2011 \\
\hline
\end{tabular}


onward (Figs. 2, 3, 4, 5, 6, 7, 8; not present at E9.5 and E10); this sector was ascribed to the prospective diagonal area, since it appeared as a thin band intercalated between ampler areas of the incipient MGE that seemed to fall into the pallidal and preoptic domains. To corroborate this analysis, we compared in alternating sagittal sections the topography of Sst cells relative to domains expressing either Dlx5 (Figs. 2f-o, 3a-d), Shh (Fig. 2t-w), Gbx2 (Fig. 3i-1) or $N k x 2.1$ (E11; Fig. 5z). Dlx5 is expressed in all subpallial domains, and certifies the source is subpallial (Bulfone et al. 1993; Eisentat et al. 1999). Shh is strongly expressed in the dorsal preoptic ventricular and mantle zones (POA1 of the Allen Developing Mouse Brain Atlas), as well as in cells that migrate selectively from there into the pallidal mantle (Pal) (Gelman et al. 2009). Gbx2 is selectively expressed in the Pal mantle (Bulfone et al. 1993; Chen et al. 2010; Flandin et al. 2010), and $N k x 2.1$ is positive in the pallidal, diagonal $(\mathrm{Dg})$ and preoptic (POA) domains, excluding the striatum (St) (Lazzaro et al. 1991; Shimamura et al. 1995; Sussel et al. 1999; Puelles et al. 2000; Flames et al. 2007; García-López et al. 2008).

The analysis of this material showed that the precocious Sst cells lie within the subpallial domain that coexpresses Dlx5 and $N k x 2.1$ (i.e., it excluded the St as a source; Figs. 2a-o, 3a-h, o, 5z). Lateral sagittal sections showed some Sst cells aligned subpially, apparently migrating tangentially in a marginal position within the Pal (Fig. 2a, f). More medial sagittal sections contained instead $S s t$ cells disposed radially within a narrow intermediate wedge of MGE mantle zone, which ends close to the ventricular zone (i.e., respecting the rostrolateral $\mathrm{Pal}$ and the caudomedial POA domains; Fig. 2b-e, g-s). This intercalated domain corresponds within our model to the Dg (Fig. 1b). This interpretation is particularly supported by comparison of these Sst cells with the Shh-expressing ventricular and mantle zones, which do not include the Dg at these section levels (Fig. 2t-w; see also inset $\mathrm{v}^{\prime}$ ).

The scenario is similar in a slightly more advanced E10.5 embryo in which we compared Sst with Dlx5 and Gbx2 (Fig. 3). The Sst population appears in a largely separate domain, the $\mathrm{Dg}$, which is intercalated between the $G b x 2+$ Pal mantle and the Gbx2- POA mantle (Fig. 3ap). The $S s t$ cells observed most laterally were again disposed tangentially in the pallidostriatal marginal zone (Fig. 3e), whereas a straightforward radial stream was progressively observed more medially (Fig. 3h).

The spatiotemporal sequence recorded at these initial stages accordingly suggests that precocious $S s t$ cells are selectively produced at the $\mathrm{Dg}$ domain, wherein they migrate towards the marginal zone. At this stage, most of them seem to advance subpially lateralward, entering the $\mathrm{Pal}$ and later the St, always in a marginal position.
Fig. 2 Lateromedial series of sagittal (adjacent) cryostat sections through the MGE at E10.5, illustrating the topography of the earliest Sst cells relative to other markers, Dlx 5 and $S h h: \mathbf{a}-\mathbf{e}, \mathbf{k} S s t ; \mathbf{f}-\mathbf{j}$, I Dlx5; m-o pseudo-color overlap of Sst and Dlx5 images for the indicated levels; p-s Sst (different specimens); t-w Shh; inset to $\mathbf{v}$ pseudo-color overlap of $\mathbf{r}$ and $\mathbf{v}$. Note the $S s t$ cells clearly occupy a restricted domain within the Dlx5-positive MGE mantle, which is intercalated between the pallidal and preoptic domains labeled with Shh signal

\section{Early telencephalic Sst cells in transverse sections}

A more precise mapping of the precocious Sst cells and their incipient tangential migration relative to the diverse subpallial domains was obtained by examining sections cut transversal to the telencephalic peduncle and the hypothalamus, i.e., the hypothalamo-telencephalic prosomeres (Puelles et al. 2012; see plane T in Fig. 1a). We examined in this way several E10.5 and E11.5 embryos (Figs. 4, 5, 6; S1), in which Sst expressing cells were compared in adjacent sections to cells expressing either Gbx2 or Shh (Figs. 4a-r, 5a-x), Nkx5.1 or Shh (Fig. 6), or Shh or $L h x 7 / 8$ (Fig. S1).

The rostralmost sections in Fig. 4 intersect the optic stalk, the preoptic area and the septal area (Fig. 4a-c, mo). Most pallidal $G b x 2$ cells lie superficial to the large ventricular/subventricular zone, mainly in the rostral half of the MGE (Pal; Fig. 4c); their number diminishes across the local paraseptal transition (PalSe) into the pallidal septum (SePal; Fig. 4a, b), and also caudalwards (Pal; Fig. 4d). The caudal pole of the Pal domain, which approaches the prospective amygdala, is still devoid of such cells (Pal; Fig. 4e, f, $\mathrm{f}^{\prime}$ ). The radially disposed $S s t$ neurons of the Dg area are observed just under the maximum of pallidal Gbx2 cells (Dg; Fig. 4i, i', j'; the insets Fig. $4 d^{\prime}, j^{\prime \prime}, p^{\prime \prime}$ correspond to a section level intermediate between I and J). Pseudocolored overlap comparison of these two markers (Fig. 4i') suggested that there are no double-labeled cells, though there are some intermixed units. The Sst cells largely are arranged radially underneath the pallidal mass of $G b \times 2$ cells (compare also Fig. $4 \mathrm{~d}^{\prime}$ and $\left.\mathrm{j}^{\prime \prime}\right)$. No Sst cells were found at more rostral section levels, where Dg continues into the septum (Fig. 4g, h), irrespective of the proximity of pallidal $G b x 2$ cells. In contrast, more caudal sections at levels where pallidal $G b \times 2$ cells diminish in number displayed a sizeable Sst population, which partly appeared intermixed with the caudalmost pallidal Gbx2 neurons (Fig. 4d, j, j'), and then massively aggregate at the marginal Pal mantle zone, partially invading as well the St domain, always subpially (Fig. 4k, 1). Caudally, similar cells are also present at the caudal amygdaloid pole of the MGE or Pal (Fig. 4, inset $1^{\prime}$ ). Interestingly, the section levels where many tangentially 


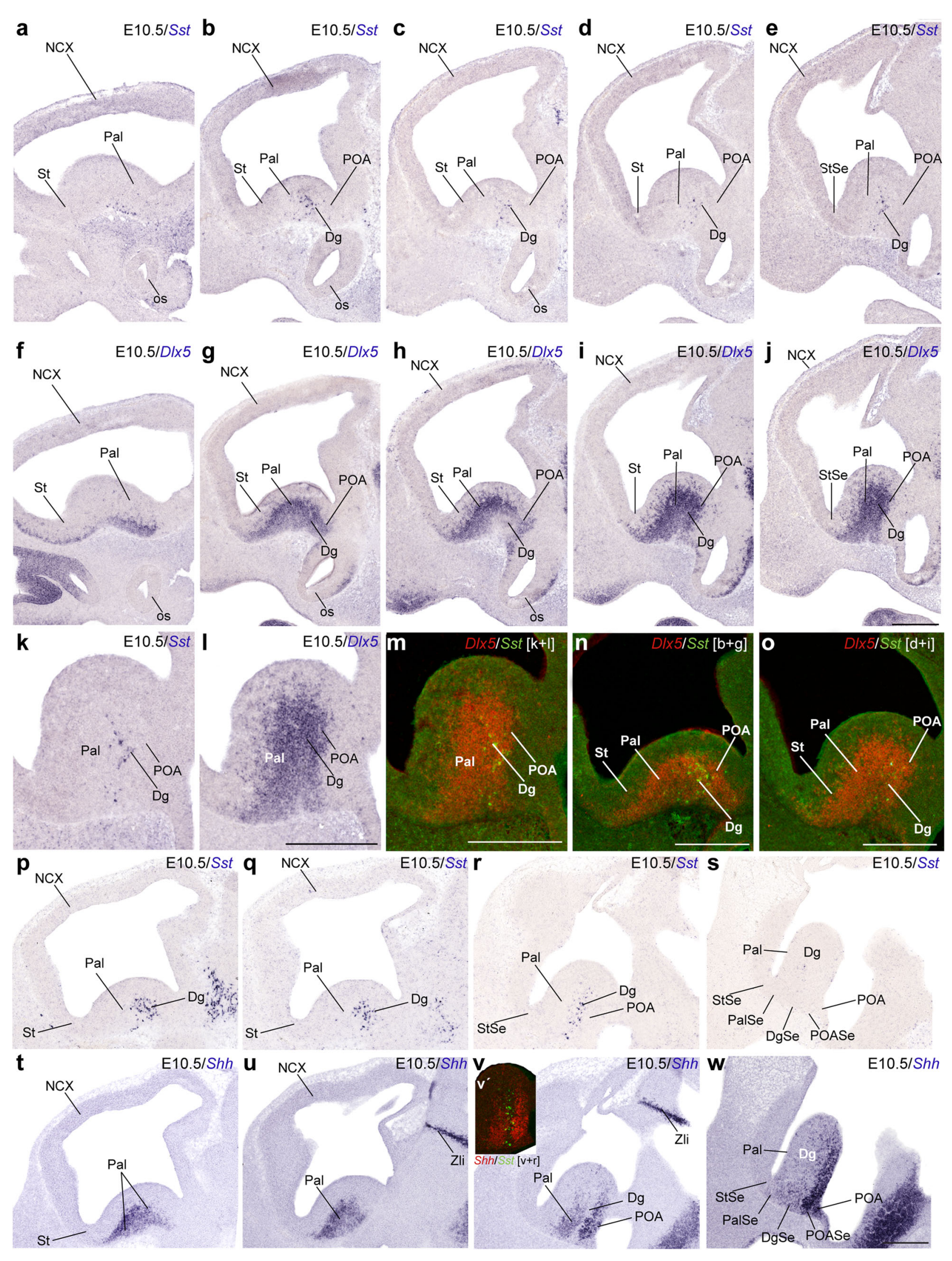



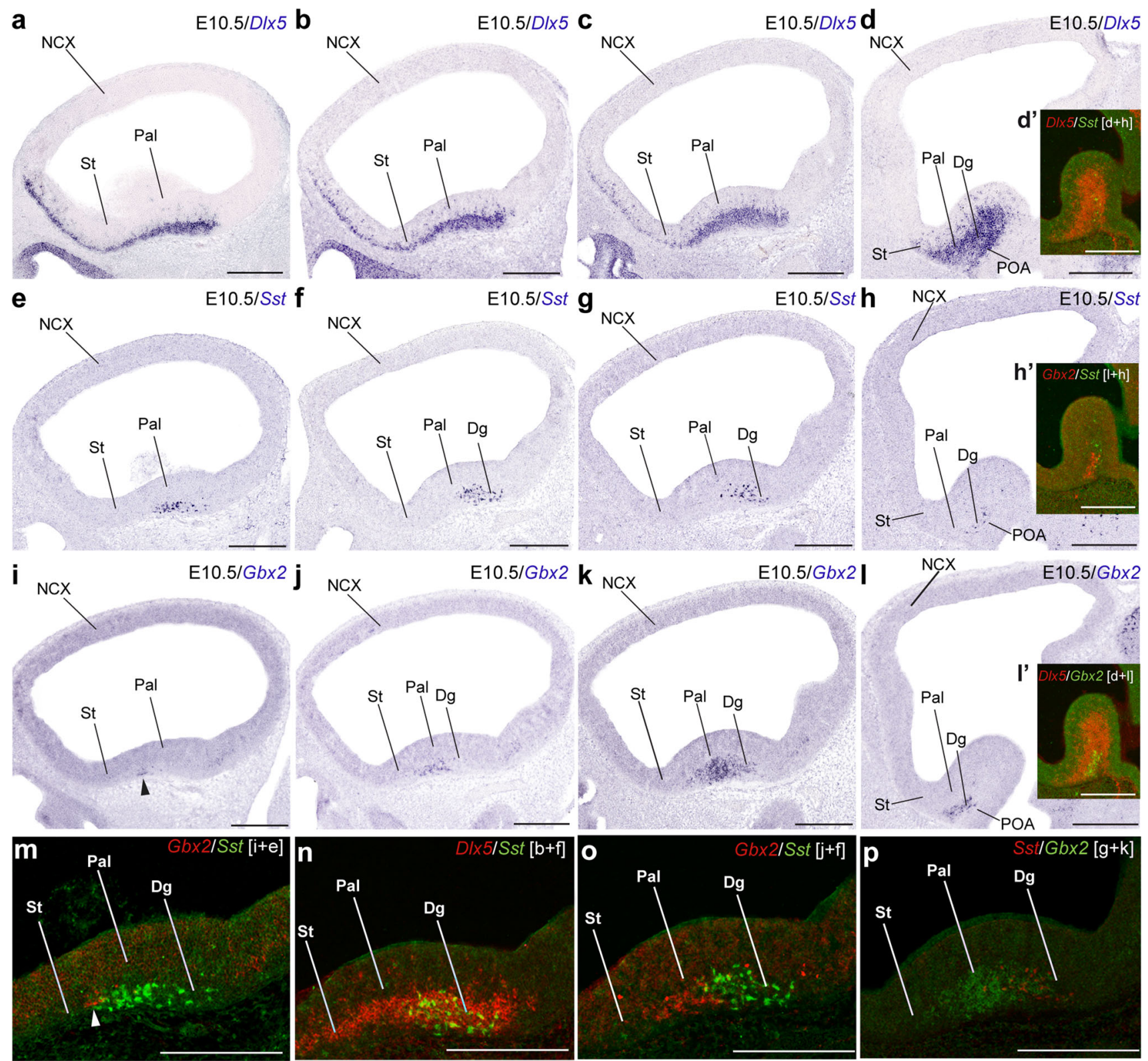

Fig. 3 Lateromedial series of sagittal (adjacent) cryostat sections through the MGE at E10.5, illustrating the topography of the earliest Sst cells relative to other markers, Dlx5 and Gbx2: a-d Dlx5; e-h Sst; $\mathbf{i}-\mathbf{l} G b x 2 ; \mathbf{d}^{\prime}, \mathbf{h}^{\prime}, \mathbf{l}^{\prime}, \mathbf{m}^{\prime}-\mathbf{p}^{\prime}$ various pseudocolor overlap comparisons;

migrating Sst cells are found are largely devoid of pallidal Gbx2 cells (Fig. 4).

On the other hand, pallidal Shh cells observed in adjacent sections have at this stage a rather rostral topography, agreeing in general with that of $G b \times 2$ cells, though the $S h h$ population is clearly more abundant and extensive rostrocaudally (Fig. 4m-r). As regards expression of Shh at the ventricular zone, there is none within the Pal domain of the MGE, whereas a strong signal was found at the part of the preoptic area that builds the medial end of the MGE the markers and levels are indicated. Note lack of overlap between Sst cells and the pallidal expression of $G b x 2$ (which does overlap with Dlx5; see n)

(Fig. $4 \mathrm{~m}-\mathrm{o}$ ); this seems to be the progenitor domain where the cells migrated into the Pal mantle originate. This lateral Shh-expressing sector of preoptic neuroepithelium is only present rostrally, consistently with the postulated ascription of the POA to the hypothalamic prosomere 2 (hp2); it limits with the $\mathrm{Dg}$ across the $\mathrm{hp} 2 / \mathrm{hp} 1$ border (Puelles et al. 2012; Fig. 1).

The Dg ventricular zone shows in contrast weak and patchy expression of Shh (Fig. $4 \mathrm{~m}-\mathrm{p}$ ), and it is unclear whether it contributes $S h h$ cells to the corresponding 
mantle domain (Dg; Fig. 4p); this includes the transitional paraseptal diagonal area lying between $\mathrm{Dg}$ and Se proper (DgSe; Fig. 4m, n). Similar weak ventricular Shh expression was found to a limited extent at the median septum (Se; Fig. 4n). On the whole, the subpallial mantle zone contains numerous $S h h$ cells, which bridge the distance between the POA/Dg sources and the postmigratory Pal domain, particularly at rostral section levels, where the Shh neurons partly overlap with presumably intrinsic pallidal Gbx2 ones (Fig. 4a-c, m-p); these cells do not penetrate subsequently the striatum (St; Figs. 5, 6). Their number decreases toward the septum, as well as caudalwards, beyond the transverse level where the Sst cells first appear (Fig. 4p-r). At these caudal levels, the Sst cells migrating through the marginal Pal adopt a position that is largely superficial to the $S h h+$ mantle stratum (Fig. $4 \mathrm{j}-1, \mathrm{p}-\mathrm{r}$ ). Curiously, the POA mantle zone does not show radial accumulation of Shh cells at all (Fig. 4m-o).

Similar transversal sections obtained at E11.5 show minimal changes (Fig. 5). The pallidal population of $G b x 2$ cells is now more extensive, reaching more caudal levels of the MGE (Fig. 5a-g). This caudal prolongation adopts a rounded shape centered in the MGE and does not invade the superficial stratum of the local mantle. Sst cells are still absent at the rostralmost levels of the subpallium. They first appear in a radial arrangement close to the $\mathrm{Dg}$ ventricular zone at the section levels that contain the maximum of $G b \times 2$ cells (Fig. 5d-g, 1-q; compare also horizontal sections in Fig. 5y, z, aa, which illustrate the restricted topography of postmitotic $S s t$ cells relative to the $N k x 2.1+$ MGE complex and the incipient migration into the St). Overlap comparison of these patterns indicates again that the Dg Sst cells lie precisely underneath and outside the Pal Gbx2 cells (insets Fig. $5 \mathrm{l}^{\prime}, \mathrm{m}^{\prime}, \mathrm{o}^{\prime}$ ), though some positive cells were observed close to the pallidal subventricular zone (small arrows; Fig. 5o, p). The caudal sections through this area illustrate again numerous Sst cells passing into the marginal Pal stratum (circumventing the central mass of Gbx2 cells), and penetrating tangentially the striatal marginal zone (Fig. 5np; see also horizontal sections in Fig. 5ac, ae, af, where pioneering invasion of the pallial cortical plate is visible as well). At these caudal levels, the radial stream of Sst cells sorting out of the Dg ventricular zone is still observable (Dg; Fig. 5n-p). It seems that the Dg source of Sst cells has expanded backward between E10.5 and E11.5. This pattern is also observable in the Shh-positive ventricular and mantle cell populations, which are essentially similar to those described at E10.5, except that the pallidal mantle shows now aggregated $S h h$ cells also at the more caudal section levels, and the POA ventricular cell source is also more extensive caudalwards (Fig. 5q-x, $\mathrm{z}, \mathrm{ab}$, ad). The preoptic mantle remains devoid of radially aggregated $S h h$ cells, but remains itself strongly positive (Fig. $5 r-x, a b$ ).
We compared in transverse sections the early distribution of $S s t$ and $S h h$ cells with cell populations expressing Nkx5.1 (Hmx3) (Fig. 6). This transcription factor was reported to be a selective marker of the preoptic area (Wang et al. 2000; Gelman et al. 2009). At 10.5, there are two quite different expression domains at rostral and caudal section levels, respectively (Fig. 6). In rostral transverse sections behind the septum, there appears a distinct marginal stratum of $N k x 5.1$ cells, which seems associated to the transition of the Dg domain into the septum, that is, to the paraseptal DgSe area and the septal SeDg area (Fig. 6a-d). Other more sparsely distributed labeled cells possibly might be ascribed to the septal SePal area (Fig. 6d). Most of these cells lie superficial to the migrating Shh cells that emerge from the POA and Dg domains and target the Pal (compare Fig. 6q-t). The rostral part of the POA1 area shows a transition into the septum (via the POASe area), which largely lacks $N k x 5.1$ cells (Fig. 6a-c). In contrast, as we proceed into more caudal transverse sections, the paraseptal Dg population disappears and a distinct preoptic $N k x 5.1$ population associated to the POA1 mantle zone (at the ventral end of the MGE) becomes apparent; this forms a distinct mantle domain that extends caudalwards with progressively fewer and deeper cells, always found underneath the Dg domain populated by $S s t$ cells (POA1; Fig. 6d-h, 1-p). The strict association of the patch of $N k x 5.1+$ cells with the $S h h+$ ventricular zone of the POA 1 area is demonstrated in the insets Fig. $6 u^{\prime}, v^{\prime}, w^{\prime}$. Overlap comparison of the $N k x 5.1$ elements in the preoptic mantle with $S s t+$ cells in the Dg area illustrates that the latter lie strictly above the $N k x 5.1+$ preoptic ones (Fig. $6 \mathrm{~d}-$ h, l-p and insets 6m', n', o'). In this specimen, we also observed sparse $S s t+$ cells at the DgSe and SeDg areas (Fig. 6i, j, k). These results support that the Sst cells are produced independently of the preoptic area, largely caudal to the paraseptal diagonal transition into the septum, and do not invade the preoptic area in their radial and tangential early migrations (see Fig. 6p; note this pattern respects the hp1/hp2 boundary).

Finally, we also compared $L h x 7-8$ expression in transverse sections with the studied $S s t$ and Shh patterns. Lhx7-8 is a general marker for Pal, Dg, and POA, thus offering a contrast with the more selective Sst and Shh signals (Grigoriou et al. 1998; García-López et al. 2008; Zhao et al. 2003). We observed that the pallidal mantle expresses massively $L h x 7-8$, occupying even the marginal stratum that is devoid of Shh cells (Figs. S1m-r). There are also many marked cells scattered in the subventricular zone of the Pal domain, particularly at rostral section levels, where these elements are relatively more numerous in the medial part of the MGE than laterally, and the corresponding medial mantle stratum is also more massively populated (Fig. S1m-o). The Dg domain, as defined by the weak and 


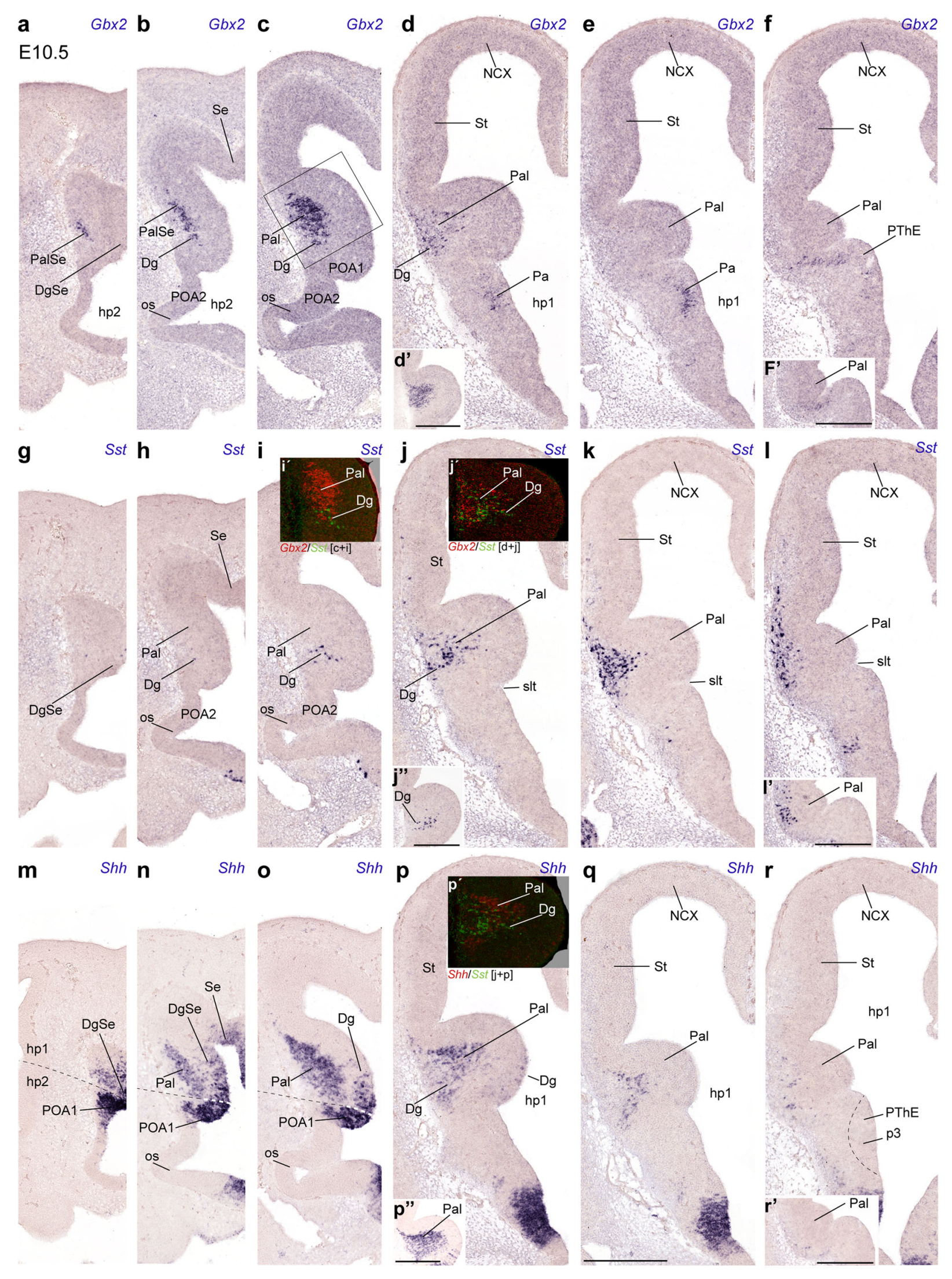


4Fig. 4 Rostrocaudal series of topologically transversal cryostat sections through the MGE (see plane in Fig. 1a) at E10.5, illustrating in correlative adjacent sections the topography of the earliest Sst cells relative to other markers, Gbx2 and Shh: a-f Gbx2; g-l Sst; inset $\mathbf{l}^{\prime}$ detail of section caudal to (l); $\mathbf{m}-\mathbf{r}$ Shh; insets $\mathbf{i}^{\prime}, \mathbf{j}^{\prime}, \mathbf{p}^{\prime}$ pseudocolor overlaps of the indicated markers and levels. Note lack of overlap of Sst cells with the Gbx2- and Shh-positive pallidal mantle elements. Pallidal Gbx2 cells occupy in general more rostral levels than Sst cells, and tend to respect the pallidal marginal stratum. Note also the incipient subpial migration of Sst cells across the marginal pallidal stratum into the striatum $(\mathbf{j}-\mathbf{l})$. As regards Shh expression, the POA1 ventricular zone appears strongly labeled, and clearly produces a migrating cell stream in the mantle that invades selectively the pallidum $(\mathbf{n}, \mathbf{o})$. The adjacent $\mathrm{Dg}$ ventricular zone shows less intense and patchy Shh signal as well, all the way to the septal end of this band, which diminishes gradientally toward the amygdaloid pole of the MGE, but does not seem to contribute to the preoptic pallidopetal cell stream in the mantle $(\mathbf{m}-\mathbf{o})$

patchy ventricular expression of $S h h$, seems to contribute likewise to this mz/vz $\operatorname{Lh} x 7-8$ pattern, though it shows a thinner positive mantle (Fig. S1o-q). At the diagonal part of the septum (median SeDg area), only a positive mantle zone was present (Fig. S1m; compare with the neighbouring pallidal part of the septum -SePal- see the inset $\mathrm{S} 1 \mathrm{~m}^{\prime}$ ), suggesting a possible tangential migration from more lateral origins. Once the POASe area is reached in the series of sections, the $L h x 7 / 8+$ mantle zone disappears (Fig. S1n). At a single section level, there was a marginal line of labeled cells at the POA2 (Shh-negative) area (Fig. S1o). More caudally, there appear instead $L h x 7 / 8$ cells in the POA1 mantle (ventral MGE), which is distinctly thinner than that present at the $\mathrm{Pal} / \mathrm{Dg}$ complex (Fig. S1p-r). The $L h x 7-8+$ Pal mantle population diminishes in cell density caudalwards, coinciding with the place where Sst cells course marginally through the Pal into the St (Fig. S1e, f, q, r). Though there is some topographic overlap between Sst and $L h x 7 / 8$ cells at the Dg domain and neighbouring Pal, many tangentially migrating Sst cells clearly do not express $L h x 7 / 8$ (compare Fig. S1d-f with $\mathrm{S} 1 \mathrm{p}-\mathrm{r})$. It is unclear whether this implies that these cells downregulate an initial postmitotic expression of $L h x 7-8$.

\section{Progress of telencephalic Sst cell populations between E12.5 and E14.5}

Migrating Sst cells streaming tangentially through the subpial part of the central subpallium start to invade the striatum and the pallium at E11.5 (Fig. 5y, aa, ad, ae, af). At E12.5, sagittal sections illustrate the arrival of many migrating Sst cells at the rostrolateral part of the telencephalic pallium either via the massive superficial subpallial migratory stream (Fig. 7a, b), or via the less populated subventricular striatal stratum (Fig. 7k), best observed in horizontal sections (small arrows; Fig. 7f-k). In contrast, medial parts of the pallium and the paraseptal and septal parts of the subpallium are devoid of Sst cells (Fig. 7c). The horizontal sections clearly show the spatial relationship between the SSpM and the presumed origin of the Sst cells, the central diagonal area territory $(\mathrm{DgC})$; the latter appears disposed as an oblique (diagonal) band of labeled cells at the back of the SSpM. The labeled diagonal population is sparse superficially at the site of the prospective diagonal band nuclei (DgC; Fig. $7 \mathrm{~d}$ ), but increases significantly at the corresponding intermediate and periventricular strata (DgC; Fig. $7 \mathrm{e}-\mathrm{g})$. In addition, less abundant $S s t$ cells apparently also course rostrolaterally at various depths through the central pallidal and striatal territories, finally incorporating into the SSpM or the subventricular pallial zone, or penetrating extensively the central striatal mantle (Fig. $7 \mathrm{e}-\mathrm{j}$ ). There are clearcut caudolateral and rostromedial boundaries of the SSpM, which possibly coincide with the limits of the central part of the subpallium versus the amygdaloid and paraseptal/ septal sectors (Fig. 1b). The amygdaloid area (consisting of both subpallial and pallial parts) is also incipiently invaded by $S s t$ cells that either stream back tangentially from the $\mathrm{DgC}$ domain, or originate locally from the amygdaloid sector of the Dg domain (DgA; asterisks in Fig. 7f, g). Sst cells invade the pallial amygdala coursing either superficially or periventricularly (large arrows in Fig. 7e-g; see also $7 \mathrm{~h}-\mathrm{j}$ ). Interestingly, the incipient globus pallidus developing within the central pallidal mantle seems to be relatively non-permissive for the reported diagonal central migration into the striatum and the cortex, so that it tends to be eschewed by the superficial and deep migrating Sst cells, and thus appears as a nearly unlabeled cell mass adjacent to the $\mathrm{DgC}$ band (GP; Fig. 7f, g). Migrating cells reach the SSpM passing all around the GP (Fig. 7e-h). The cell stream connecting the caudal $\mathrm{DgC}$ and the $\mathrm{DgA}$ with the neighbouring SSpM is particularly dense (Fig. 7e-g); data will be shown below suggesting that this locus relates to the prospective Sst-positive part of the central amygdaloid nucleus. Sst cells are most dense subpially at the primordium of the olfactory tuberculum, whose superficial corticoid layer is not yet distinguished at E12.5 (TO; Fig. $7 \mathrm{~d}-\mathrm{g}$ ). Sst cells moving past the striatum clearly invade the primordium of the prepiriform cortex, before reaching the neocortical marginal layer ( $\mathrm{PirCx}, \mathrm{Cx}$; Fig. 7h-k); fewer cells enter subpially the rostrolateral frontal pallium via independent subpial and subventricular routes (Fig. 7a, b, f-k).

At E13.5 the pioneering Sst cells reach the convexity of the cortical mantle, where they appear mainly dispersed among the cortical plate and subplate cells; the developing cingular and hippocampal cortical areas, as well as the medial amygdala, are devoid of labeled cells (Figs. 8a-j, 9a-c, g, h). At this stage, the earlier subventricular migratory stream entering the cortex has largely 


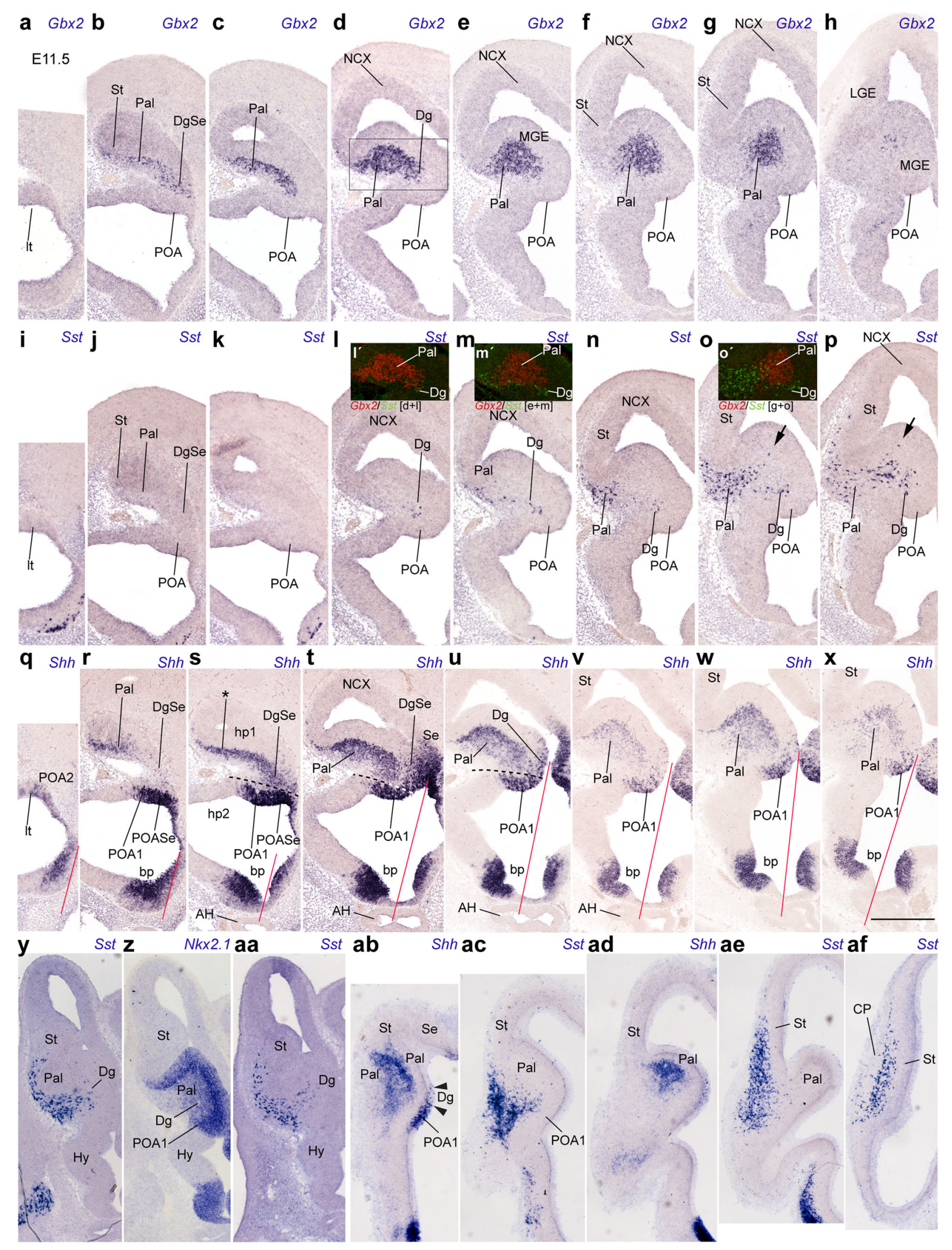


4Fig. 5 Rostrocaudal series of topologically transversal cryostat sections through the MGE (see plane in Fig. 1a) at E11.5, illustrating in correlative adjacent sections the topography of the $S s t$ cells relative to other markers, Gbx2, Shh, and $N k x 2.1: \mathbf{a}-\mathbf{h} G b x 2 ; \mathbf{i}-\mathbf{p} S s t ; \mathbf{q}-\mathbf{x} S h h$; $\mathbf{y}$, aa, ac, ae, af $S s t ; \mathbf{z} N k x 2.1 ; \mathbf{a b}$, ad $S h h ;$ insets $\left.\mathbf{I}^{\prime}, \mathbf{m}^{\prime}, \mathbf{o}^{\prime}\right)$ pseudocolor overlap of the indicated markers and levels. The red straight lines entered into panels $\mathbf{q}-\mathbf{x}$ indicate the midplane. At this stage, the Gbx2-positive pallidal population extends farther caudalwards, but still essentially does not overlap with the Sst cells at the Dg mantle; isolated Sst cells that apparently do overlap with Pal are marked with arrows in $\mathbf{0}$, p. Note progression of subpial migratory invasion of the striatum by $S s t$ cells $(\mathbf{n}-\mathbf{p}, \mathbf{y}, \mathbf{a a}, \mathbf{a c}, \mathbf{a e}, \mathbf{a f}) . S h h$ labeling of POA1, Dg, and Pal agrees with the description in Fig. 4; the pallidal $S h h$-positive mantle respects the marginal stratum occupied by migrating Sst cells (ab-ae)

disappeared, though some dispersed Sst cells are still visible within this stratum (arrow in Fig. 8a). The major tangential migratory course is represented by the SSpM observed at the pial surface of the subpallium (not shown); from there $S s t$ cells proceed into the pallial mantle lying under the olfactory cortex (SSpM; PirCx; Fig. 8a). The majority of labeled cells approaching the SSpM course behind the globus pallidus, bypassing laterally the internal capsule, whereas fewer cells apparently join this stream passing through the deep corridor passing across the pallidal and striatal mantle (Fig. 8a). In Fig. 8, we enclosed with a black line the areas we estimated to be subpallial, to aid the description of Sst cell populations with pallial versus subpallial topographies. The densest pallial Sst cells were found at the prepiriform/piriform cortex (mainly layer III; Figs. 8a-h, 9a) and in the pallial amygdala (mainly amygdalohippocampal area; AHi; Figs. 8c, d, 9a). The olfactory bulb primordium is devoid of $S s t$ cells (Figs. 8i, j, $9 \mathrm{j}, \mathrm{k})$.

The central diagonal histogenetic domain $(\mathrm{DgC})$ shows its characteristic oblique band of dense Sst cells placed ventromedial to the globus pallidus (central pallidal subdomain) and dorsolateral to the preoptic area (both largely Sst-negative; DgC, GP; POA; Fig. 8b-e). The periventricular part of this band arches over the internal capsule, consistently with the subsequent position of the supracapsular BST (bed nucleus of the stria terminalis) complex. The observed dense aggregates of $S s t$ cells clearly represent the medial (diagonal) part of the supracapsular BST (BSTMsc), since the lateral BST counterpart belongs to the periventricular pallidum (BSTMsc; Fig. 8a-d; see overall map in Figs. 1b, 10q, r, 12c, d show the distinction between Sst-positive BSTM and Sst-negative BSTL at E14.5 and E15.5, respectively). Ventral to the level where the internal capsule penetrates the subpallium (closer to the olfactory tuberculum), the caudal end of the supracapsular BSTM arch extends into the amygdala, where we observe the dense radially migrated $S s t$ cells of the amygdaloid BST complex (BSTA) and the associated diagonal part of the central amygdala (CA), as well as other $S s t$ cells that migrated tangentially into the pallial amygdala, invading mainly the prospective basal and cortical region, and the amygdalo-hippocampal area, but eschewing the medial amygdala (AHi; Fig. 8e-g). On the other hand, the rostral end of the supracapsular BSTM arch reaches rostromedially the paraseptal part of the diagonal BSTM complex (BSTPs); some labeled cells apparently disperse from here into the neighbouring preoptic area (BSTMps, POA; Fig. $8 \mathrm{c}-\mathrm{f}$ ). The intermediate stratum of $\mathrm{DgC}$ found underneath the anterior commissure builds the substantia innominata; it only contains sparse Sst cells (SI; Fig. 8f, g); such cells become slightly more abundant at the corresponding superficial $\mathrm{DgC}$ stratum, occupied by the horizontal nucleus of the diagonal band (HDB; Fig. 8h-j).

At E13.5, the striatal mantle shows a dispersed population of labeled cells, whose density markedly increases near the pial surface (future ventral striatum), and reaches a maximum at the SSpM stream, which lies now deep to the incipient olfactory tuberculum (SSpM; Fig. 8i, j; compare 8a). Coronal sections indicate that this dense subpial stratum is also present, but thinner, at the nucleus accumbens (paraseptal striatum) and the neighbouring striatal septum (not shown). Comparison in adjacent sagittal sections of the expression patterns of $S s t$ and $N k x 2.1$ (a general marker at early stages of Pal, Dg and POA) illustrates at E13.5 that $S s t$ cells are practically absent in the globus pallidus (GP; Fig. 9a, b, d, e), and only the ventral pallidum and the periventricular pallidal around the GP contain some Sst cells, which presumably are passing through into the striatum and pallium (Fig. 9c-1). In contrast, the Dg periventricular zone appears characterized by dense $S s t$ cells, which form the BSTM primordium (BSTM; Fig. 9c, $\mathrm{g}, \mathrm{h}$ ); this locus is characterized by rather weak, or absent, Nkx2.1 expression at E13.5 (Fig. 9f, j, k; see also pseudocolor overlaps in $\mathrm{i}, \mathrm{l})$. Also the corresponding paraseptal and septal diagonal ventricular zone showed distinctly less $N k x 2.1$ signal than the neighbouring paraseptal and septal pallidal and preoptic domains (Fig. 9j-1). This change in molecular background distinctly separates a pallidal $N k x 2.1+/ S s t$ - domain (with strong $N k x 2.1$ ) from the $N k x 2.1+/ S s t+$ diagonal domain (with weak $N k x 2.1$ ).

One day later, at E14.5, coronal and horizontal sections illustrate various more advanced aspects of the static and migrating $S s t$ populations. Whereas $S s t$ cells do not yet enter the olfactory bulb or the anterior olfactory area, the tangentially migrating cells now incipiently colonize the insular, frontal, orbital, infralimbic and anterior cingulate cortical areas (Fig. 10a, s), as well as most of the striatal mantle, the latter in a decreasing gradient toward the ventricular zone (St; Fig. 10b-j, p-s). The piriform cortex primordium lying deep to the lateral olfactory tract appears densely penetrated by $S s t$ cells, a differential characteristic 


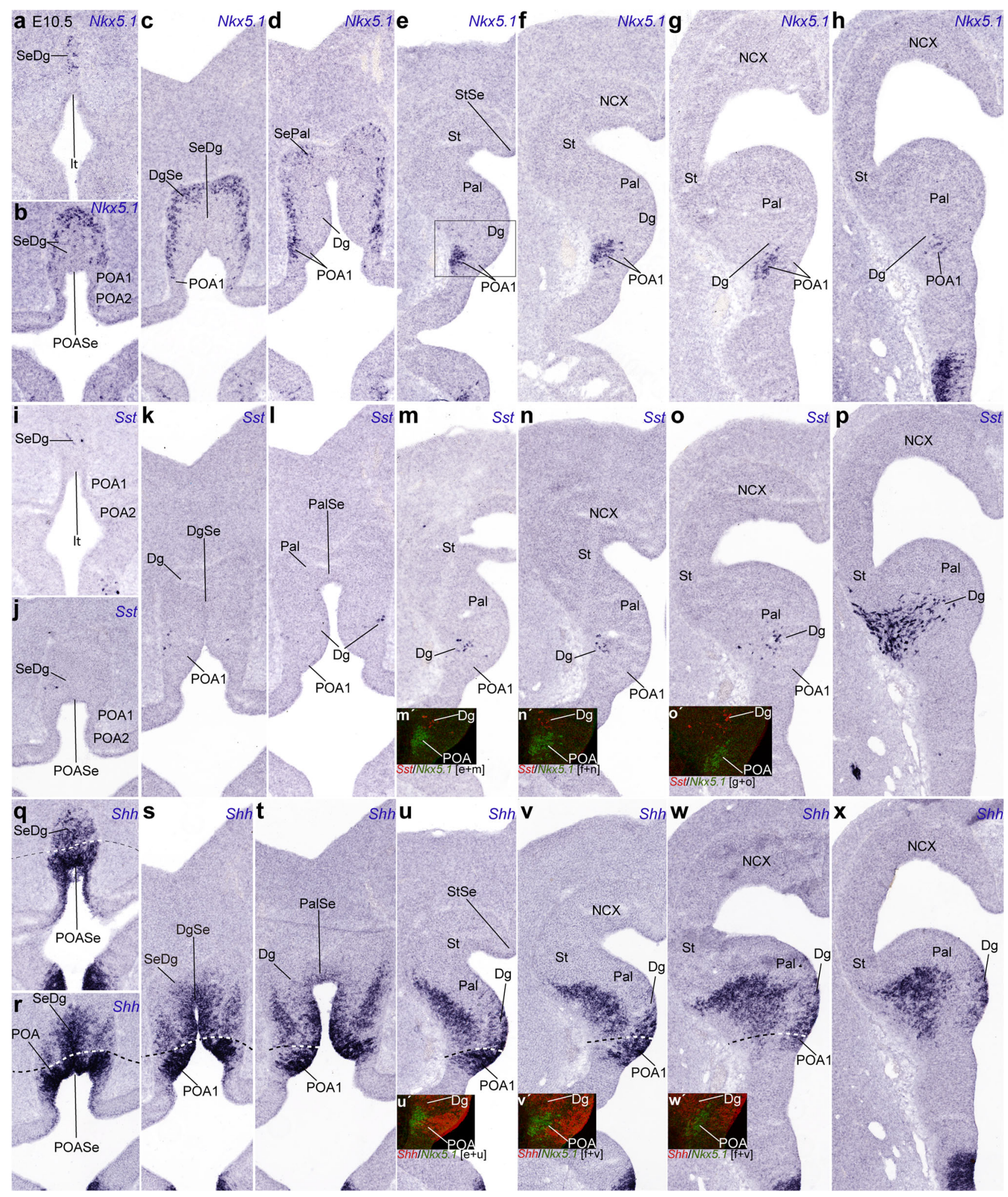

with respect to the overlying, less populated claustro-insular complex and other parts of the cortex (PirCx, lot; Fig. 10b-n, o-s); the intervening clearcut boundary separates the newly postulated ventropallial and lateropallial derivatives (see Puelles 2014). Just medially to the lateral olfactory tract, the subpallial subpial stratum is 
4Fig. 6 Rostrocaudal series of topologically transversal cryostat sections through the MGE (see plane in Fig. 1a) at E10.5, illustrating in correlative adjacent sections the topography of the Sst cells relative to other markers, Nkx5.1 and Shh: a-h Nkx5.1; i-p Sst; q-x Shh; insets $\mathbf{m}^{\prime}-\mathbf{o}^{\prime}, \mathbf{u}^{\prime}-\mathbf{w}^{\prime}$ pseudocolor overlap of the indicated markers and levels. There are abundant $N k x 5.1$ cells in the preoptic and diagonalseptal neighborhoods (a-d), as well as in the POA1 mantle layer (eh), without significant overlap with Sst cells. In p, a particularly favourable section plane demonstrates the continuity of Sst cells originated selectively at the Dg domain with the incipient migratory phenomenon at the marginal stratum, without apparent implication of the pallidal domain. Note some Sst cells are adjacent to the POA1 mantle, without intermixing (insets $\mathbf{m}^{\prime}-\mathbf{o}^{\prime}$ ). In contrast preoptic Nkx5.1 mantle cells are continuous (and partly mixed) with the pallidopetal Shh-positive migrating cells in the mantle (insets $\mathbf{u}^{\prime}-\mathbf{w}^{\prime}$ ). The ventricular zone of Dg clearly expresses patchily Shh (in a septoamygdaloid decreasing gradient; $\mathbf{s}-\mathbf{x}$ )

still full of Sst cells, which correspond to the SSpM stream traversing the prospective olfactory tuberculum (SSpM; Fig. 10b-f, o-q). These subpial Sst cells also extend medialward into the subpallial paraseptal areas next to the septum, but only across the accumbens area (paraseptal St) and the diagonal-septal area (DgSe or paraseptal $\mathrm{Dg}$ ); interestingly, this does not occur at the intervening pallidoseptal area (PalSe or paraseptal Pal) (Acb; PalSe; DgSe; Fig. 10b-e; p). Immediately caudomedial to the olfactory tuberculum, the number of Sst cells present at the horizontal part of the diagonal band has increased, but remains less abundant than at the olfactory tuberculum, suggesting absence of a tangential migratory route at this locus (DB; Fig. 10f-h). The DB formation is radially continuous with deeper labeled cells that form a sublenticular population medially and caudally to the conspicuously negative globus pallidus; this intermediate stratum of the central diagonal area corresponds to the classic substantia innominata (DgC/SI; GP; Fig. 10f-i). The corresponding periventricular stratum contains the diagonal supracapsular BSTM (BSTMsc), continuous rostromedially with the corresponding paraseptal sector (BSTMps). The latter's labeled cells contrast with the completely unlabeled prethalamic eminence behind it (BSTMps; PThE; Fig. 10f-h, p, q). The supracapsular BSTM can be followed caudolaterally over the internal capsule, next to the negative GP (BSTMsc; Fig. 10h, i, q), until it reaches its amygdaloid end (BSTMa; Fig. $10 \mathrm{j}-1$, p). The dense population of Sst cells in the BSTMps, BSTMsc, and BSTMa contrasts sharply with the absence of such cells in the adjacent pallidal part of the BST, identified by us as BSTL (BSTL; Fig. 10r, s; see also Fig. 11 and supplementary Fig. S2a, b). Irrespective of its low $N k x 2.1$ expression level, the BSTM clearly lies within the Dlx5-expressing subpallium (at its border), whereas the neighboring supracapsular migration stream that vehiculates hypothalamic Otp-positive neurons into the amygdala passes just medial to the BSTM, outside the Dlx5-positive subpallium, within a thin periventricular pallial corridor that connects the pallial amygdala with the peduncular hypothalamus (Fig. 3a-c; García-Moreno et al. 2010; Morales-Delgado et al. 2011; Puelles et al. 2012).

The BSTMa in its turn connects ventrolaterally with the densest part at this stage of the diagonal radial migration stream, which approaches superficially the caudal end of the SSpM, passing behind the GP; this very dense radial stream of labeled cells is first found superficially, lateral to the GP, and then laterally to the internal capsule, behind the GP. According to observations at later stages (see below), many of these cells form definitive nuclear derivatives, irrespective that others may enter the SSpM and continue tangentially into the cortex or the striatum. These local radial mantle derivatives of the amygdaloid part of the diagonal area correspond to the primordium of the lateral central amygdala, found next to the BSTMa (CA; Figs. 8f, g, 10g-1, p), and, more superficially, to the primordium of the classic magnocellular preoptic nucleus. The latter term is a clear misnomer (since the locus is well outside the preoptic area, and the POA does not produce $S s t$ cells), which leads us to propose renaming it the magnocellular diagonal nucleus, or DgMC (this agrees with its topography close -but deep- to the horizontal DB nucleus; DgMC; Figs. 8h, i, 10g-h, o). At section levels caudal to the GP, the dense CA primordium contains an unlabeled anteroposterior stream of cells in its interior, pointing toward the anterior amygdalar area, which we believe corresponds to the migratory stream of the nucleus of the lateral olfactory tract (NLOTm; Fig. 10j, k; Remedios et al. 2004).

At E14.5 the pallial amygdala lying lateral and caudal to the CA displays ventrally a large ovoid area with sparse Sst cells, which we believe is the primordium of the basolateral amygdaloid nucleus, and dorsally to it, deep to the PirCx, there appears a cap-like area full of Sst cells, which corresponds to the prospective lateral amygdaloid nucleus, as indicated by data at subsequent stages (BL; L; Fig. 10j-1, o-q). The medial amygdala instead shows few labeled cells (MA; Fig. 10j-n, o), whereas the area where the prospective basomedial and corticoid nuclei form contains a moderate amount of Sst cells (untagged; Fig. 10k-n, o).

\section{Progress of telencephalic Sst cell populations between E15.5 and E16.5}

We also analyzed the expression of Sst in sagittal sections at E15.5 (Fig. 11) and horizontal sections at E16.5 (Fig. 12). In general, we noted a progressively wider dispersion of Sst cells within the pallium, notably including incipient invasion of caudomedial areas such as entorhinal cortex, subiculum, and hippocampus (ERh; S; Hi; Figs. 11a-f, 12c-h). Sst cells appear broadly dispersed in 


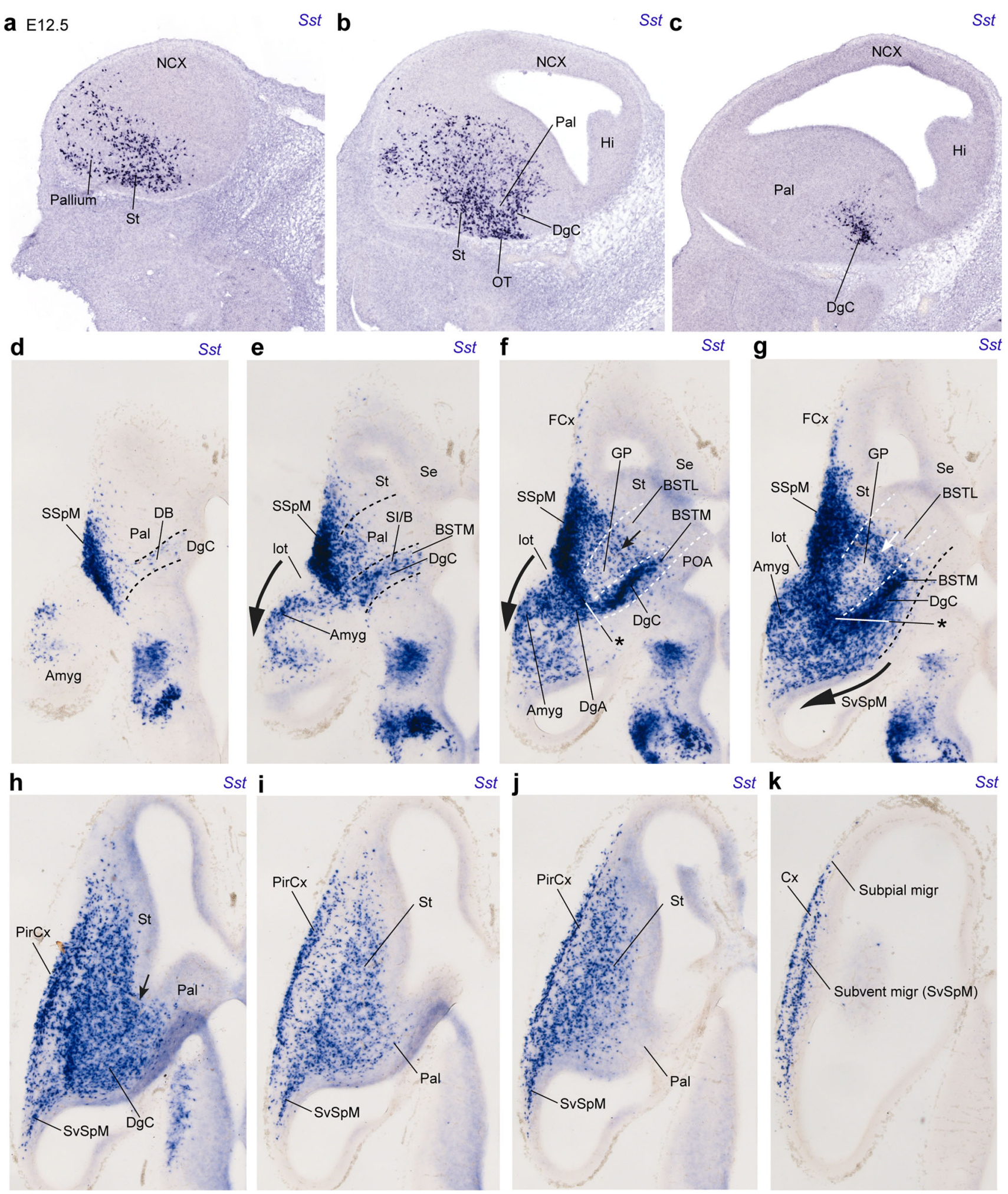

all regions of the neocortex (Figs. 11a-f; 12f-h), though there remains a distinctly larger population in the ventral anterior olfactory area, the olfactory tuberculum and the prepiriform and piriform areas (AOA, OT, PirCx; Figs. 11a-f, 12a-h); such olfactory cortex cells subsequently largely populate the adult layer III (see 
4Fig. 7 Examples of sagittal and horizontal sections through E12.5 embryonic brains, showing the migratory dispersion of $S s t$ cells at this stage. a-c Lateral to medial set of selected sagittal sections showing the progress of the invasion of the olfactory tuberculum (OT) and striatum (St), as well as the incipient subpial tangential invasion of the pallium (mainly olfactory and insular cortex primordia); the medialmost section c shows a restricted topography of Sst cells within a central area of the Dg, eschewing the larger pallidal mantle, except at its marginal stratum seen in (b). d-k Ventrodorsal series of horizontal sections, illustrating the septo-amygdaloid dimension of the studied distribution of Sst cells; the striatal, pallidal, and diagonal domains are delimited tentatively one from another by oblique white or black dash lines. The marginal stratum of the whole olfactory tuberculum is occupied by the dense subpial subpallial migratory stream, where $S s t$ cells stemming from the Dg domain are seen to arrive (SSpM; Dg; dg). Rostrally, labeled cells extend into frontal cortex ( $\mathrm{FCx}$ ); caudally large subpial and subventricular streams of Sst cells invade nonhomogeneously the pallial amygdala, beyond the DgA region of the subpallial amygdala (large arrows in $\mathbf{e}-\mathbf{h}$ ). Note as well the existence of Sst cells migrating subventricularly across the pallidum into the striatum (small arrows; $\mathbf{f}, \mathbf{g}$ ). The piriform cortex primordium (largely prospective layer III) appears strongly labeled (PirCx; $\mathbf{h}-\mathbf{j})$

supplementary Figs. S3a, b), suggesting that at the observed intermediate developmental stages there is still a relatively immature state of these allocortical areas. There are scarce labeled cells in the pallial subventricular zone, whereas the marginal stratum contains many cells (Figs. 11a-f, 12e-h). At section levels through the insular cortex, there appears a line of deep aggregated Sst cells that are aligned with the lateropallial claustrum (CL; Fig. 12h; see Puelles 2014). This claustral aggregate is separated by label-free white matter from a parallel line of densely labeled cells next to the striatum. The latter cells seem to have invaded deep nuclear derivatives of the ventral pallium that are associated to the external capsule, namely the recently identified bed nucleus of the external capsule (BEC; Puelles 2014), previously known as 'reservoir' (Bayer and Altman 1991). The BEC seems continuous caudally with the densely $S s t$-labeled primordium of the lateral amygdaloid nucleus (LA), a larger triangular pallial aggregate also held to derive from the ventral pallium (Medina et al. 2004). The continuity of these two formations is partly interrupted by passing fibers of the posterior limb of the anterior commissure (CL, BEC; Fig. 11a; CL, BEC, LA, ac; Fig. 12e-h). Ventral to the LA, the basolateral amygdaloid nucleus retains its original ovoid aspect and sparse population of $S s t$ cells, particularly laterally (BL; Figs. 11a, a $\mathrm{a}^{\prime}, 12 \mathrm{c}, \mathrm{d}$ ). This primordium is surrounded dorsally and caudally by a relatively dense migratory stream of labeled cells apparently spreading out of the BSTMa (asterisk in Figs. 11a, a', 12d, e). This intraamygdaloid stream surrounds the BL and connects rostrally and dorsolaterally with the base of the LA (see L; Figs. 11a, 12e-g), and caudoventrally with the amygdalopiriform area and the basomedial nucleus (APi, BM; Figs. 11a, a' $12 \mathrm{c}-\mathrm{e})$. Cells from this stream also extend medialwards into the amygdalohippocampal area, possibly connecting there with a separate periventricular migratory stream (BM, AHi; Figs. 11a, a', b, c, 12c, d). In contrast with these at least transiently well-populated areas of the pallial amygdala, the anterior amygdala, and the medial amygdalar nuclei, including the Shh-positive posteroventral medial nucleus (green in Fig. 11b), show only few dispersed Sst cells (AA, MePV, MA; Figs. 11b-d, 12b). The posterolateral and posteromedial amygdalar cortical nuclei show a slightly more abundant population of $S s t$ cells (PLCo, PMCo; Fig. 12b).

As regards the subpallium, the striatum population of $S s t$ cells develops over these stages (E15.5, E16.5) a nearly mature appearance. The distribution remains gradiental along the radial dimension extending from the olfactory tuberculum, past the ventral striatum, into the main body and its subventricular zone at E15.5, (St, OT, VSt; Fig. 11a-f), but seems denser and more uniform at E16.5 (St; Fig. 12c-h). In contrast, there are few Sst cells in the fundus striati (under the tag ' $\mathrm{l}$ ' in Fig. 12g, h). Interestingly, horizontal sections illustrate a relatively high density of $S s t$ cells along the IPAC primordium (interstitial nucleus of the posterior limb of the anterior commissure), at the transition between dorsal and ventral striatum regions (IPAC; Fig. 12d-f). This aggregate encloses the label-free posterior limb of the anterior commissure and limits caudomedially with the largely unlabeled pallidal intermediate stratum forming the GP (GP; Fig. 12e-h).

Found along the radial dimension of the Pal domain extending past the GP into the subpial olfactory tubercle, the ventral pallidum (VPal) shows increased presence of Sst cells compared to GP, though always less than the neighbouring striatal and diagonal superficial domains (GP, VPal, VSt, SI; Figs. 11e, f, 12c, d). The periventricular pallidal stratum (identified by NKX2.1-immunoreaction in Fig. 11) is represented by the BSTL primordium, found lateral to the Sst-positive BSTM (which is largely negative for NKX2.1). The supracapsular BSTL is practically devoid of Sst cells, particularly at its subventricular zone, though some Sst cells characterize the local mantle deep to the internal capsule (BSTLsc; Fig. 11b-d). The related pallidal paraseptal area, identified here as SePal, similarly shows a number of dispersed Sst cells within the deeper mantle zone, next to its negative subventricular zone (SePal; Fig. 11e, f). These deep elements may represent remnants of the earlier deep migration stream across the $\mathrm{Pal}$ into the striatum, and are in fact clearly continuous with the striatal population (Fig. 11c, d). Finally, it is possible that the amygdaloid end of the pallidal mantle is represented by the medial part of the CA complex, which appears scarcely populated by $S s t$ cells, in contrast with the richly populated lateral part of CA (CeL, CeM; Fig. 11b). This difference was also observed in the adult CA 

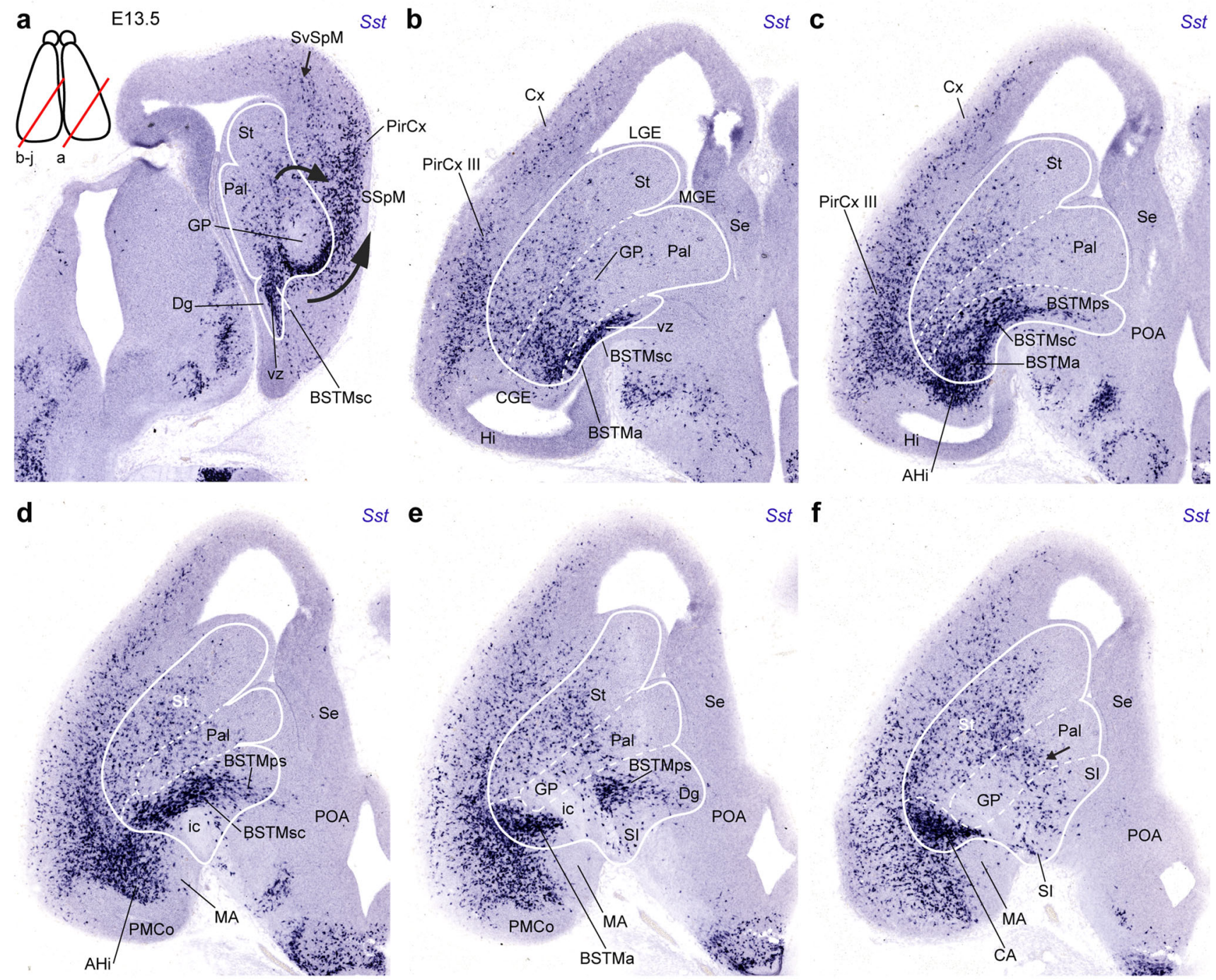

Sst $\mathbf{f}$

Sst
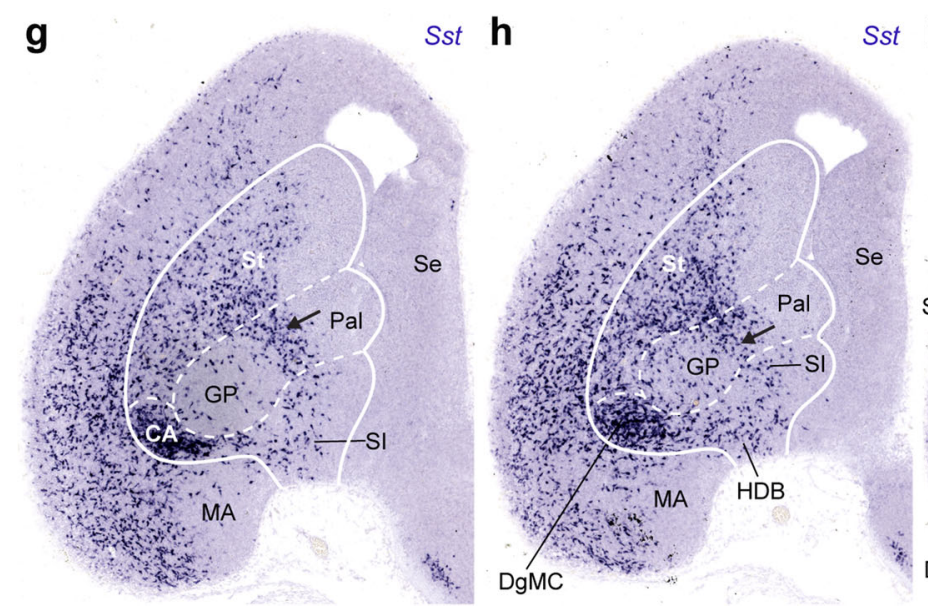

Sst i
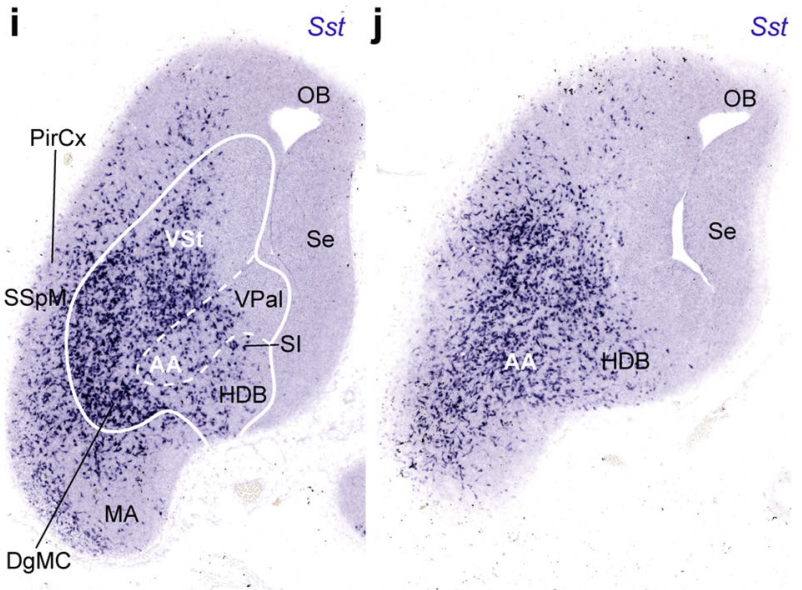

(Fig. S3b). Collateral data mining in the Allen Developing Mouse Brain Atlas revealed that $I s l l$ expression in the CA seems restricted to its medial part $(\mathrm{CeM})$.
The diagonal domain appears segregated radially into deep, intermediate, and superficial components, like the pallidal domain. All of them contain abundant Sst cells. 
4Fig. 8 Examples of oblique transversal sections through an E13.5 embryonic brain, showing the migratory dispersion of $S s t$ cells at this stage. The plane of section is indicated by red lines in the inset to a (the section in a corresponds to the right hemisphere, while the other sections illustrate the opposite side). a The obliquity of this section (see inset) aligns the strongly labeled Dg source of Sst cells (Dg) with the path of their migration deep and superficial to the eschewed globus pallidus (curved arrows GP), finally converging at the SSpM (at the olfactory tuberculum), and advancing into the olfactory cortex (PirCx), as well as into subpial and subventricular streams targetting cortical pallium (SvSpM). b-j The oblique septoamygdaloid section plane obtained in these images (compare inset at a) is aligned with the diagonal domain, and in general with the three evaginated subpallial domains (delineated as a whole by a white contour line, with dashed internal limits); the series starts caudally close to the lateral ventricle (b; see the CGE, LGE, MGE bulges) and progresses into the olfactory tuberculum at $\mathbf{i}, \mathbf{j}$. The maximal density of labeled cells coincides with the Dg ventricular zone $(\mathbf{b}, \mathbf{c})$ and associated periventricular stratum, which forms the supracapsular arch of the medial bed nucleus striae terminalis complex (BSTMsc) over the internal capsule (ic; $\mathbf{d}, \mathbf{e}$ ); beyond this level, the amygdaloid end of the Dg arch displays a very dense aggregate of Sst cells, identified first as the amygdaloid BSTM nucleus (BSTMa) and then as the CA (the primordium of the central amygdaloid nucleus, lateral part) (e-g). The latter is continuous superficially-close to the SSpM and the OT-with the diagonal magnocellular nucleus (DgMC; $\mathbf{h}, \mathbf{i}$; this was classically misidentified as 'preoptic magnocellular nucleus'). The other end of the Dg arch constitutes the paraseptal region of the BSTM, which limits with the septum (Se) and the preoptic area (POA) (BSTMps; $\mathbf{c}-\mathbf{e}$; compare Fig. 1b). Ventral to the globus pallidus there are dispersed Sst cells within the substantia innominata and the horizontal part of the diagonal band formation (SI; HDB; e-j). The pallial amygdala shows substantial invasion by $S s t$ cells of its amygdalo-hippocampal and basolateral/basomedial areas (AHi; c-g); in contrast, the medial amygdala (MA) and the posteromedial corticoid area (PMCo) largely remain devoid of these cells $(\mathbf{d}-\mathbf{i})$

The periventricular derivative is the BSTM, which appears largely as a thin strongly $S s t$-positive band in sagittal sections (Fig. 11), but seems broader in horizontal sections (Fig. 12). In fact, horizontal sections suggest that this population of Sst cells is dual, being composed by an anterolateral thin band of large and strongly Sst-expressing neurons (marked by an asterisk in Fig. 12h) and a caudomedial broader parallel band of smaller and less strongly labeled cells; outside the asterisk-marked cells in Fig. 12h). This dual constitution was observed as well at the amygdaloid and paraseptal ends of the BSTM formation (unlabeled in Fig. 11; BSTMa, BSTMps; Fig. 12), and probably accounts for some of the detailed subdivisions described there in the adult (see "Discussion"). The BSTM complex can be subdivided along the septo-amygdaloid axis into paraseptal, central (supracapsular), and amygdaloid parts (BSTMps, BSTMsc, BSTMa; Figs. 1b, 11b-f, 12c-h). The paraseptal BSTM progressively diminishes in cell number toward the negative preoptic area (e.g., Figs. 11f, 12c). Adjacent laterally to the BSTMa, there is the very dense and highly labeled primordium of the lateral part of the CA complex (CeL; Fig. 11b), which may be understood as representing the last periventricular diagonal formation along the septoamygdaloid axis. The $\mathrm{CeL}$ is continuous superficialward with the DgMC nucleus (usually named 'magnocellular preoptic nucleus' in the literature, though it obviously lies outside the preoptic region), which represents the amygdaloid intermediate Dg stratum, found lateral to the substantia innominata (DgMC; Fig. 11b). At the intermediate stratum of the central Dg area, there appears immediately under the internal capsule a small caudomedial extension of the unlabeled GP, whose cells are distinctly NKX2.1-immunoreactive; we identified the two GP parts identifiable in this material as corresponding to the prospective external and internal pallidal segments (EGP, IGP; Fig. 11b-d; note the estimated pallidal boundaries are highlighted by black lines); it is unclear whether the IGP originates primarily at the Pal or Dg domains; in any case, it shows few Sst cells, which is a Pal-like feature. Underneath the IGP there appears the substantia innominata (SI), another constituent of the central diagonal intermediate stratum, which displays a dispersed population of Sst cells (SI; Fig. 11b-f). At the superficial stratum of the diagonal domain there appears the diagonal band, whose horizontal nucleus shows a contingent of $S s t$ cells (HDB, DB; Figs. 11b-f, 12a-c). The medial septal surface also shows a Sst-positive population, which may correspond to the prospective vertical diagonal band nucleus (Se; Fig. 12ce).

\section{Discussion}

\section{Diversity of subpallial sources of tangential migrations and the associated terminology problem}

In classical models, the subpallium was composed exclusively of striatal and pallidal parts. In recent times, the subpallium model was expanded to include the preoptic area (previously ascribed to the hypothalamus; see Shimogori et al. 2010; Puelles et al. 2010) and the hemispheric stalk (peduncular) region or diagonal area (Fig. 1a, b); the latter was known previously either as substantia innominata, or as anterior entopeduncular area; Bulfone et al. 1993, 1995; Puelles et al. 2000, 2004, 2013). Among the molecular characteristics that unify these subpallial regions is the overall early expression of Dlx family, Mashl and Arx genes (Puelles et al. 2004; Shimogori et al. 2010). Analysis of gene markers that label differentially a particular subpallial ventricular zone, such as $N k \times 2.1$ in the pallidum, stalk region and preoptic area, and Shh in the POA1 part of the preoptic area (Fig. 1b), indicated that all major subpallial divisions extend from the septum to the amygdala, along the oblique septo-amygdaloid axis (Swanson and 

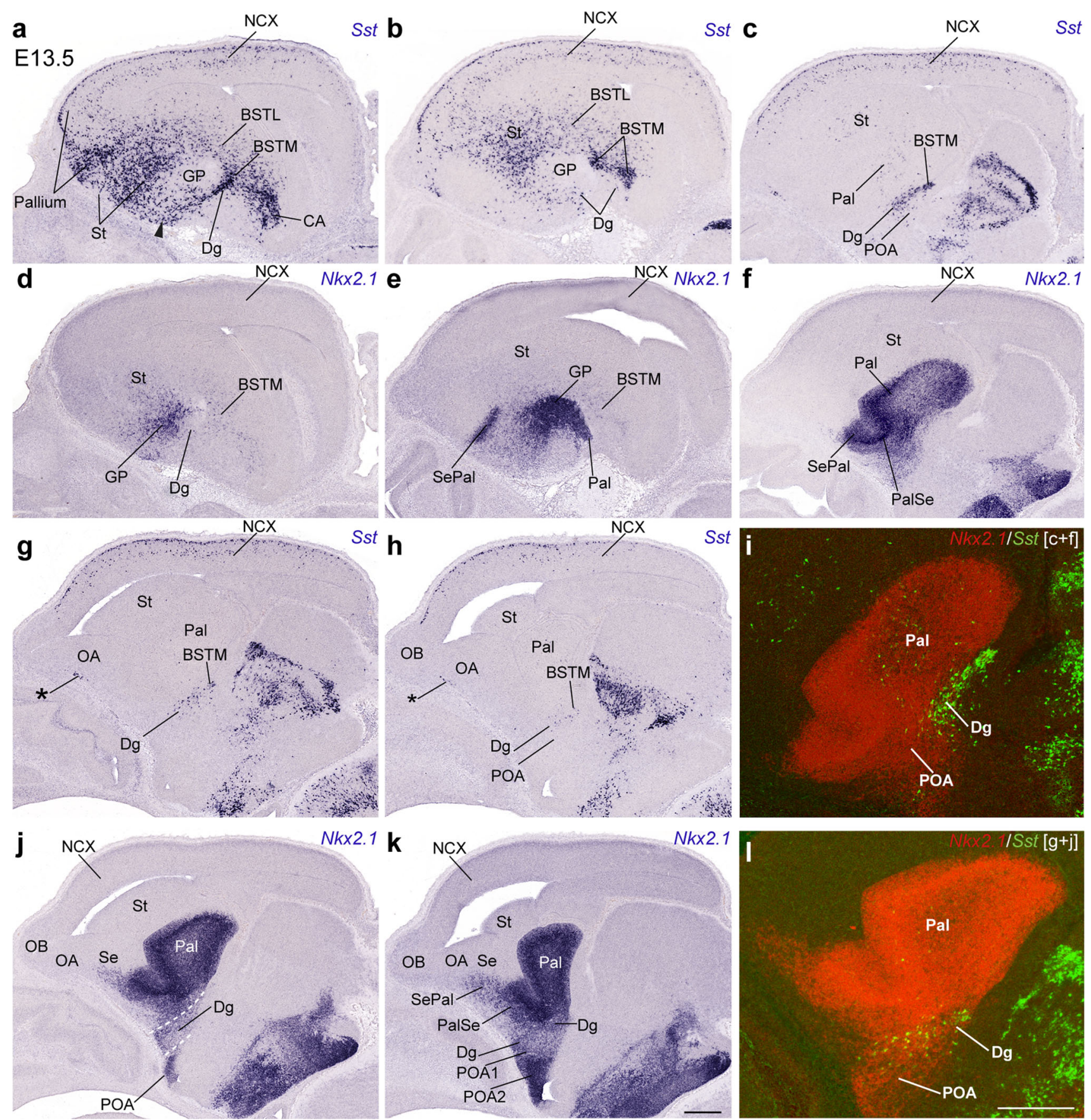

Fig. 9 Examples of sagittal sections through an E13.5 embryonic brain, showing more advanced migratory dispersion of $S s t$ cells $(\mathbf{a}-\mathbf{c}$, $\mathbf{g}, \mathbf{h}$ ), and the relationship of the Dg radial domain with the domain of expression of $N k x 2.1(\mathbf{d}-\mathbf{f}, \mathbf{j}, \mathbf{k}) ; \mathbf{i}, \mathbf{l}$ pseudocolor overlap of both

Petrovich, 1998; Puelles et al. 2000, 2013; Flames et al. 2007; Medina and Abellan 2012). The apparent caudal pole of this subpallial complex represents the caudal ganglionic eminence, ascribed to the amygdala, to which may be added the preopto-hypothalamic transition area (CGE, POH; Fig. 1a, b; Bulfone et al. 1993; Puelles and Rubenstein markers at the indicated levels. Note that at this stage, the Dg ventricular zone and at least part of its periventricular BSTM formation fall outside the domain of expression of $N k x 2$.1. Many Sst cells have invaded the isocortical plate at superficial and deep strata

1993; Xu et al. 2004, 2008; Butt et al. 2005; Fogarty et al. 2007; Sousa et al. 2009; Lee et al. 2010). Note the lateral ganglionic eminence (LGE) largely corresponds to the striatal domain, whereas the medial ganglionic eminence (MGE) encompasses the pallidal domain, the diagonal area, and part of the preoptic area (Fig. 1b). 
Flames et al. (2007) mapped at least 18 molecularly distinct progenitor domains at the subpallial ventricular/subventricular zone (Fig. 1c; note these are mostly aligned parallel to the septoamygdaloid axis). It was concluded that these domains might represent as many independent sources of specific neuronal types, which originate partly a diversity of tangentially migrating populations, and partly discrete radially stratified populations of the local mantle zone (i.e., a theoretical minimum of $18 \times 3=54$ cell populations, counting periventricular, intermediate and superficial strata at each subpallial domain; e.g., lateral bed nucleus striae terminalis, globus pallidus, and ventral pallidum plus pallidal olfactory tuberculum, within the pallidum). This conceptual background significantly qualifies the earlier simpler concepts of the lateral, medial, and caudal ganglionic eminences (LGE, MGE, and CGE), which were initially thought to represent homogeneous histogenetic entities. Indeed, classic embryologic studies had simplistically assimilated the striatum to the LGE, and the pallidum to the MGE.

The subpallial region that occupies the telencephalic stalk area, intercalated between the preoptic area and the pallidum, was recently renamed diagonal area $(\mathrm{Dg})$ in the Allen Developing Mouse Brain Atlas (online since 2009) and Puelles et al. (2013). This region is stretched like its neighbours along the septo-amygdaloid axis (Fig. 1b, c). Its intermediate stratum formed by dispersed neurons was classically identified as the 'substantia innominata' (which contains the cholinergic neurons of Meynert's basal magnocellular nucleus, among other elements, and merges laterocaudally with the 'sublenticular extended amygdala'). The Dg subpial stratum is occupied by the 'diagonal band nuclei' (horizontal and vertical), which lie interstitial to the diagonal (amygdaloseptal) tract and end at the medial septum; these nuclei also display in late embryos and the adult a prominent cholinergic population (Zaborszky et al. 2012; Allen Developing Mouse Brain Atlas). Various reports concluded that these cholinergic neurons, dependent on $L h x 7 / 8$ and IslI are not produced locally within the Dg domain (understood as medial extended amygdala, or MGEcv area; Zhao et al. 2003; Elshatory and Gan 2008; Medina and Abellan 2012); moreover, they do not share origins with other telencephalic cholinergic neurons presumedly produced at the preoptic area. Interestingly, our data reveal that ventricular and mantle expression of $L h x 7 /$ 8 is strictly restricted to the Pal and Dg areas, whereas the preoptic region only shows a few positive cells in its mantle, possibly migrated in from the Dg (Fig. S1). We believe this interpretation is consistent with corresponding data shown by García-López et al. (2008; their Figures 1C, 4D; note also the neurons expressing ChAT illustrated therein only overlap the $\mathrm{Dg}$ region). In our opinion this weakens the hypothesis of a preopto-commissural origin of cholinergic neurons (since the differentiation of these cells needs $L h x 7 / 8$ signal; Zhao et al. 2003). Here we merely wish to emphasize that whatever their origin may be (see also below our discussion of Pombero et al. 2011), these cells selectively mature within the diagonal band nuclei, as well as within the substantia innominata, both of which are components of the $\mathrm{Dg}$. The diagonal histogenetic area also encompasses a periventricular stratum that includes the medial part of the stria terminalis complex (BSTM; note the adjoining BSTL part-which differentially expresses Isl1 [Allen Developing Mouse Brain Atlas]-is instead pallidal; Fig. 1d). The Dg was characterized by Flames et al. (2007) as a molecularly distinct areal component of the MGE, identified as the pMGE5 subdomain (see Fig. 1b, c). This domain was the only 'pallidal' (meaning MGE) area that expressed the transcription factor Er81 (Flames et al. 2007; their Figure 2D); it also shows ventricular patches of Shh signal which contrast with the massive POA expression (Flandin et al. 2010; present results). As mentioned above, the pallidal BSTL region expresses differentially Isll, compared to the diagonal BSTM.

The Dg was previously named 'anterior entopeduncular area' in early reports on the prosomeric model (AEP; e.g., Bulfone et al. 1993; Puelles and Rubenstein 1993; Rubenstein et al. 1994). The AEP name has since been used widely, though it proved to be imprecise (and thus inconvenient), since it suggests an isolated cell population interstitial to the medial or lateral forebrain bundles, rather than a complete radial (ventriculo-pial) histogenetic domain, as was intended. Puelles and collaborators thus came to regard their own term as obsolete. In the search for a better alternative name, the momentary absence of markers distinguishing this area from the pallidum led to the idea that this area could be seen as a part of the Pal, or, at least, of the MGE. Alternative names accordingly employed in recent literature include 'caudal and medial MGE' (Nery et al. 2002; Legaz et al. 2005), 'central and ventral MGE' (Fogarty et al. 2007), 'anterior peduncular area' (García-López et al. 2008), 'ventral MGE' (Flandin et al. 2010, 2011), and 'caudoventral MGE' (Bupesh et al. 2011a, b; Medina and Abellan 2012). This excess of options generates by itself considerable semantic confusion, since none of these authors provided a subpallium map showing the precise location and extent of this area. We think that all these names are similarly inconvenient. First, because the axis of reference for the diverse positional descriptors used remains undefined and vague (probably the 'central' and 'caudal' terms allude to the arbitrary anteroposterior sequence of coronal section levels -the axis of the microtome- which is devoid of true morphologic value in the prosomeric model, but they might refer instead to the oblique, more realistic septoamygdaloid axis), and, secondly, because the precise position of the 


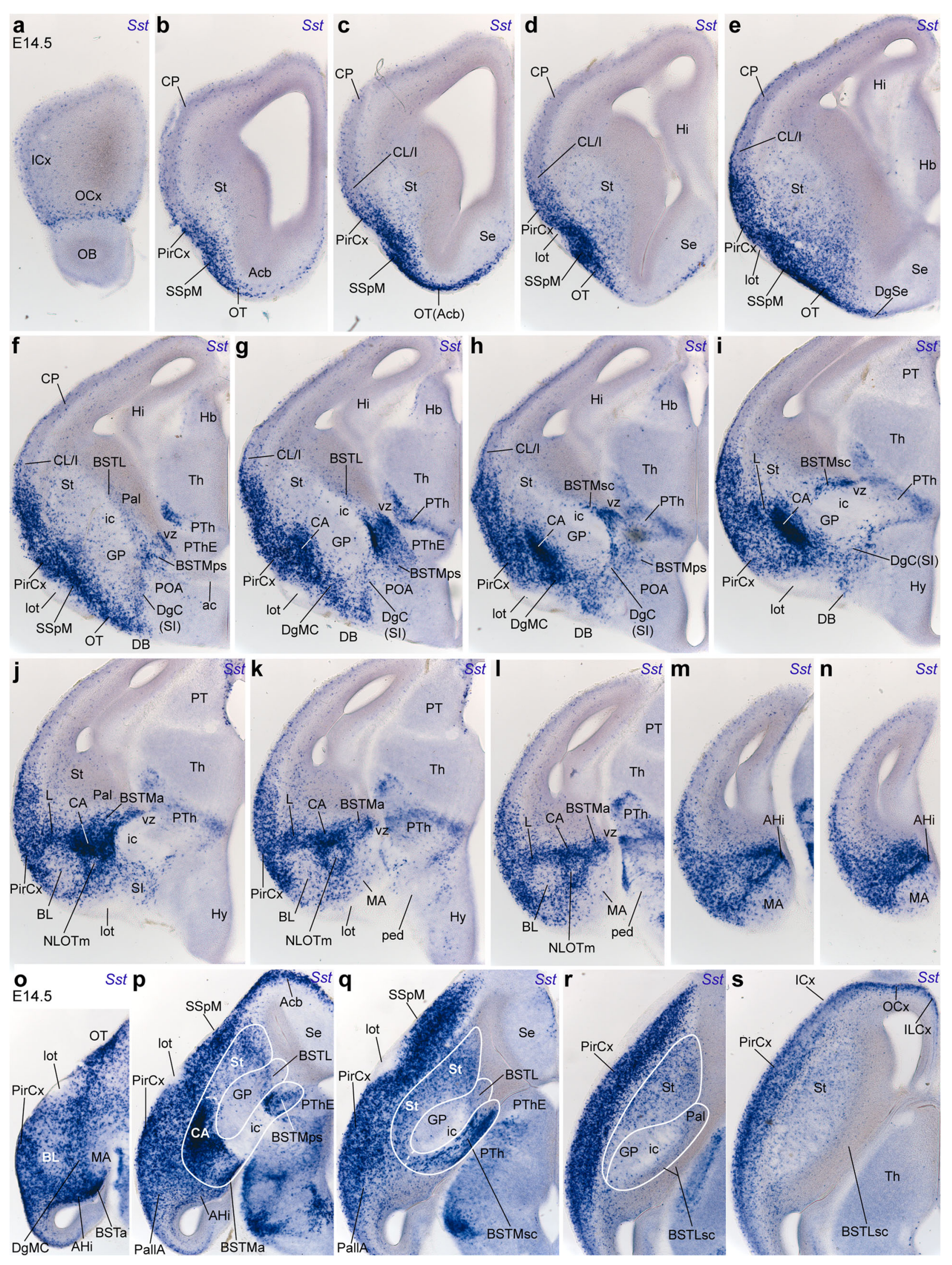


4Fig. 10 Examples of transversal (a-n) and horizontal (o-s) sections through E14.5 embryonic brains, showing the migratory dispersion and increasing differentiative stabilization of $S s t$ cells at this stage. an The rostral sections clearly display the superficial subpallial migratory stream advancing from the olfactory tuberculum, just deep to the lateral olfactory tract into the deep stratum of the olfactory cortex and then largely subpially into the insula and isocortical plate (SSpM; lot; CL/I; CP; a-f); some cells enter medially the septum (Se). The striatal primordium still shows more cells superficially than next to the internal capsule (scarce $S s t$ cells at subventricular levels). The pallidum is practically devoid of labeled cells at the globus pallidus, but shows some small elements within its periventricular (supracapsular) BSTL nucleus (GP; BSTL; f-i). As regards the Dg domain, the periventricular BSTM arch is cut obliquely in this plane of section; we see first the paraseptal component, which approaches the crossing of the anterior commissure and the preoptic area (BSTMps; so-called 'anterior' BST; f-h); the caudal end, composed by the amygdaloid lateral CA and DgMC nuclei, appears close by, internally to the position of the lateral olfactory tract (CA, DgMC; $\mathbf{g}$, h); both elements are soon united by the intermediate supracapsular portion of the BSTM complex (BSTMsc; $\mathbf{h}-\mathbf{j}$ ), at shortly thereafter we also see the labeled amygdaloid BST nucleus (BSTMa; $\mathbf{j}-\mathbf{l}$ ); the radial continuity of the Dg ventricular zone with its pial surface is populated throughout by Sst cells dispersed in the substantia innominata, converging at the brain surface upon the diagonal band nucleei (vz; SI; DB; $\mathbf{f}-\mathbf{l})$. Caudolaterally to the CA there appears the pallial amygdala, including the strongly labeled lateral nucleus and the poorly labeled basolateral nucleus (L, BL; i-l) and the strongly labeled amygdalo-hippocampal area $(\mathrm{AHi} ; \mathbf{m}, \mathbf{n})$. The medial amygdala (MA) only contains scattered labeled cells. o-s These horizontal sections offer a complementary view of the same anatomic details distinguished in transversal sections

'medial' or'ventral' area along the dorsoventral dimension also seems unclear (no defining landmarks). Confusingly, the locus of this area is often implied to be circumscribed to a limited areal spot (most authors identify it only at a standard coronal section level), though the model shown in Fig. 1b, c suggests that the relevant distinct stalk area actually extends all the way from the septum into the amygdala (Puelles et al. 2013; see text of Figure 1d). Accordingly, the position within the MGE complex of this specific area and the developmental phenomena associated to it probably result imperfectly understood by most readers.

Curiously, Flames et al. (2007) resolved minimally the molecular distinction of the stalk $(\mathrm{Dg})$ histogenetic area relative to the pallidum, observing that the former selectively expresses ER81 (Etv1) and lacks Couptfl signal, whereas the opposite is true for the pallidum. Present data illustrate a remarkably precise correlation of Sst neurons with the mantle of this domain; García-López et al. (2008) previously underlined the restricted presence at the same locus of calbindin-positive neurons, streaming tangentially into the pallial amygdala in a similar way as we observed Sst cells. Therefore, it is both possible and convenient (and is supported by the present results) to have a distinctive name for the stalk area, leaving aside the old term AEP, as well as the vague descriptors of its position within the
MGE. The recently updated prosomeric model (L.P., reference atlases developed for the Allen Developing Mouse Brain Atlas; http://www.developingmouse.brain-map.org; Puelles et al. 2012, 2013) aimed to resolve this problem by proposing for the stalk area the alternative term diagonal area $(\mathrm{Dg})$; this conveys the correct topographic linear extent along the oblique hemispheric stalk and septoamygdaloid axis, and is based on a well-known classic concept and related identifiable surface landmark, the diagonal band (i.e., the subpial diagonal band formation generally forms a surface relief that separates the flat preoptic surface from the olfactory tuberculum, which represents the neighboring subpial portion of the Pal and St). This name refers to the adult region and corresponding characteristic cell populations, irrespective whether these are produced or not within the Dg proper (e.g., case of the cholinergic cells commented above).

In summary, the narrow diagonal radial histogenetic domain defined at the hemispheric stalk (Dg; Fig. 1d) is traversed orthogonally by the medial and lateral forebrain bundles, and encompasses the diagonal band nuclei superficially (jointly with the diagonal band tract), the innominate/magnocellular basal populations at the intermediate stratum (with the ventral amygdalofugal tract), and the medial bed nuclei of the stria terminalis complex (BSTM) periventricularly (with part of the stria terminalis tract). At its septal end, the Dg is continuous with the medial septum via the vertical limb nucleus of the diagonal band. At its opposite amygdalar end, our data suggest that the Dg finishes at the amygdalar BST nucleus (BSTA) and the associated lateral part of the central nucleus (CeL; see below).

\section{Antecedents of the Sst cell migration}

In this report, we study the apparent radial origin and subsequent wide tangential migratory distribution of $S s t$ cells in the mouse telencephalon between E10.5 and E16.5. In the final distribution such cells populate within the pallium the whole cerebral cortex (a well-studied point we do not need to examine in detail), the claustrum and the pallial amygdala, showing scarce contributions to the olfactory bulb. Sst cells also invade the subpallium, though differentially. They are massively present in the lateral part of the central amygdaloid nucleus, as well as in the medial part of the bed nucleus stria terminalis complex, both of which are understood here as intrinsic (non tangentially migrated) derivatives of the diagonal area (see Suppl. Fig. 4; Puelles et al. 2013, and reference atlases of the Allen Developing Mouse Brain Atlas, online since 2009); there is also a labeled subpopulation of striatal interneurons, many labeled cells throughout the olfactory tuberculum, and some weakly labeled cells within the pallidal 


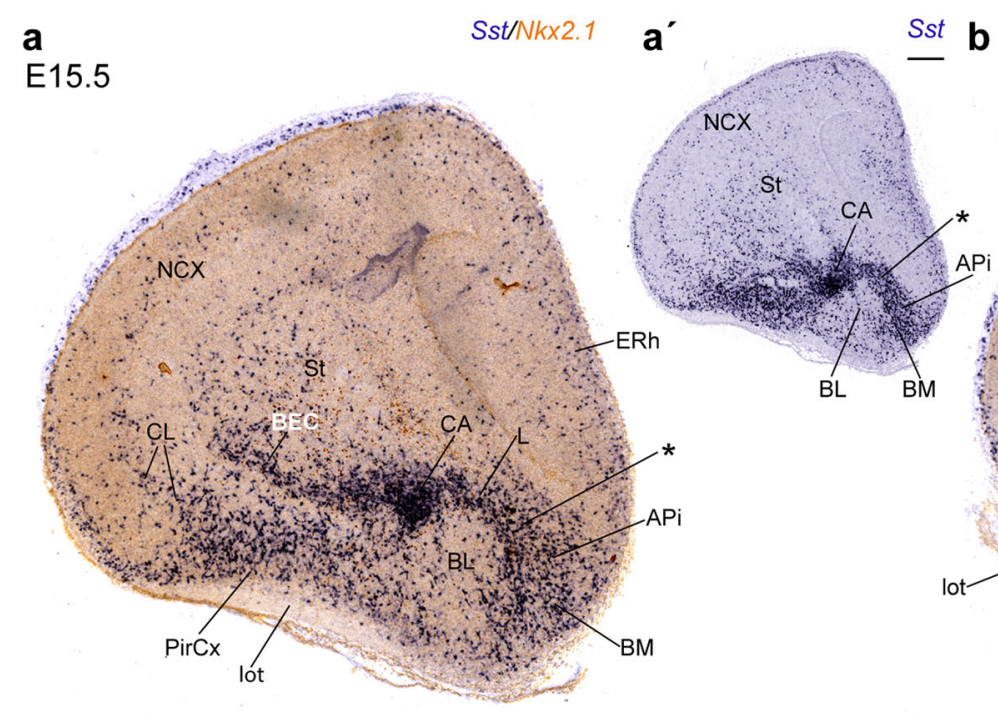

Sst b

\section{c}

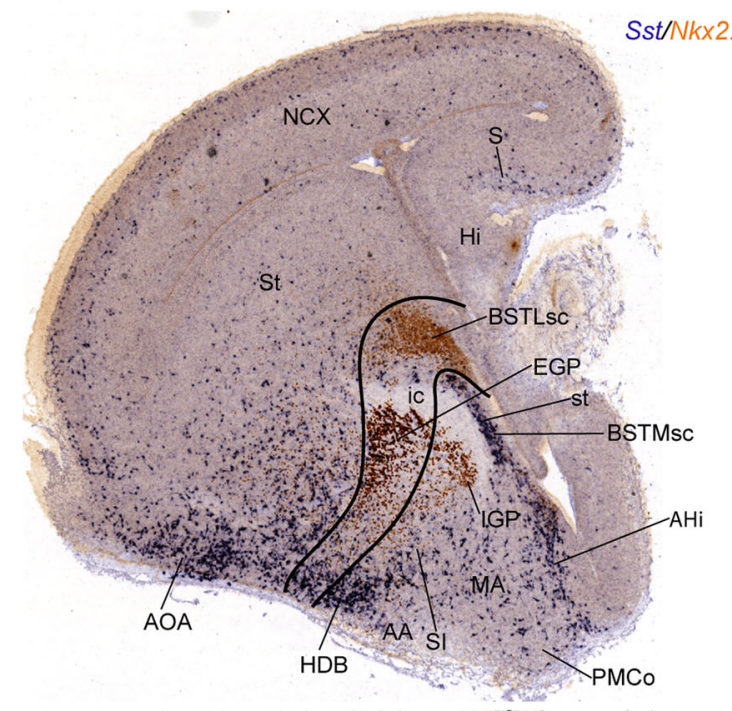

e

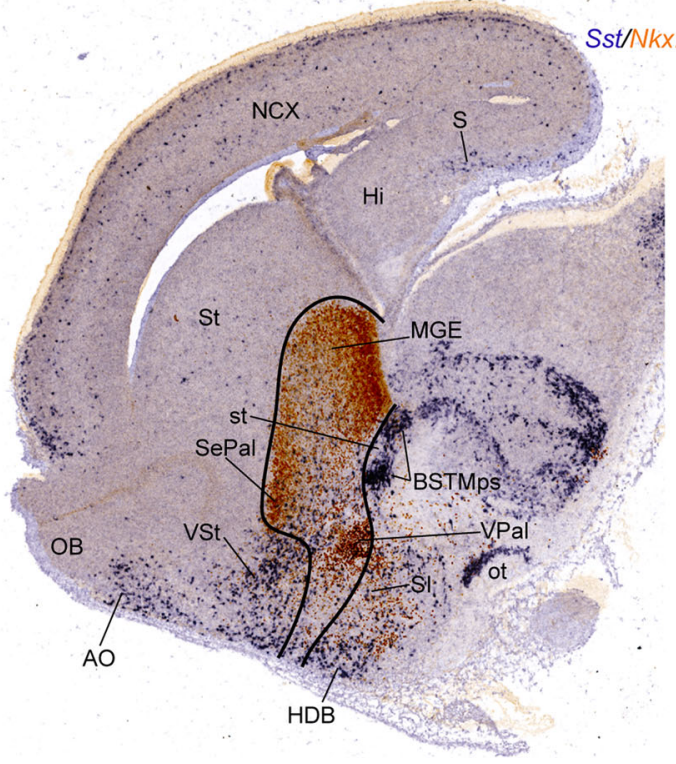

Sst/Nk $\times 2.1$

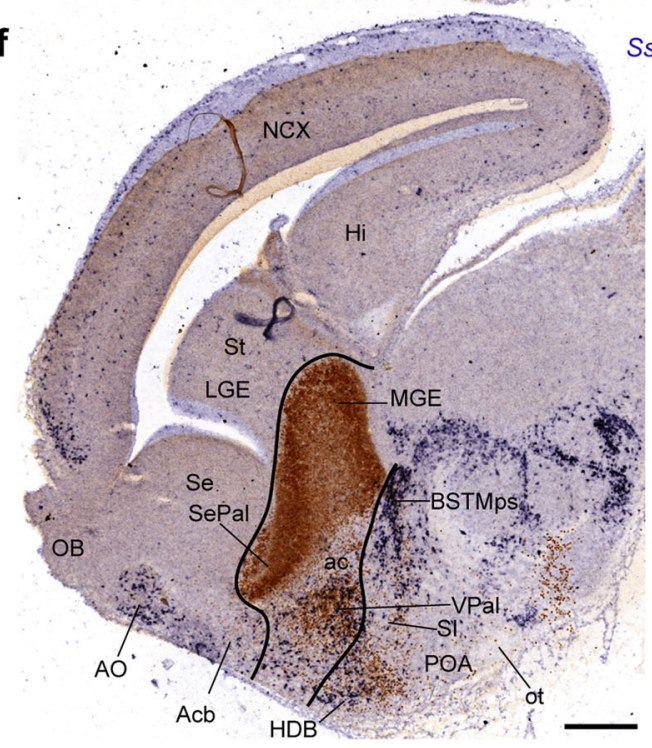


4Fig. 11 Digitally superposed images of adjacent sagittal sections at 6 section levels through an E15.5 embryonic brain, showing the distribution of Sst cells relative to the NKX2.1-immunoreactive domain (inset $\mathbf{a}^{\prime}$ shows a section lying lateral to a, reacted only for $S s t$ ISH). (a, $\left.\mathbf{a}^{\prime}\right)$ Lateral sections pass through the pallial amygdala, laterally to the globus pallidus; the central lateral amygdalar nucleus is densely labeled (CA; prospective $\mathrm{CeL}$ ); note sparse Sst cells within the neighboring basolateral amygdalar nucleus (BL) and more abundant cells at the lateral and basomedial amygdalar nuclear primordia (L, BM); migrating Sst cells have reached the entorhinal cortex, but not yet the hippocampus ( $\mathrm{ERh} ; \mathrm{Hi}$; in $\mathbf{b}, \mathbf{d})$. Labeled cells also aggregate more rostrally within the bed nucleus of the external capsule (BEC). b-d Section b shows, superposed in green, the image of $S h h$ expression within the medial posteroventral amygdaloid nucleus, a site with scarce $S s t$ cells. These more medial sections also intersect the striatum (dispersed labeled cells in a ventrodorsal gradient); next to it appears the globus pallidus (GP) and the supracapsular lateral BST nucleus (BSTLsc), both of which are NKX2.1 immunoreactive and poor in Sst cells. The pallidal radial domain ending superficially at the olfactory tuberculum (with many labeled cells), and limiting at the ventricular zone with the Sstpositive Dg domain, is tentatively enclosed by black lines in c, d. These lines suggest that the NKX2.1-immunoreactive GP is divided topographically into an external (pallidal) portion and an internal (diagonal) counterpart (EGP, IGP; b-d). The diagonal complex (Dg) includes the densely labeled periventricular BSTMsc formation, the IGP, the NKX2.1-immunoreactive substantia innominata (with relatively few intermixed $S s t$ cells) and the superficial horizontal nucleus of the diagonal band (HDB; this has as many labeled cells as the olfactory tuberculum and also contains some NKX2.1-immunoreactive cells). e, f At these more medial section levels, we reach the medial end of the supracapsular BSTM arch (BSTMsc), moving into its paraseptal portion (BSTMps), next to the anterior commissure (ac) and the preoptic area (POA)

BSTL. In contrast, Sst cells eschew rather selectively the globus pallidus and the preoptic area. Our results suggest that the earliest, and possibly the main, origin of telencephalic Sst neurons is the diagonal area, which is a longitudinal hemispheric stalk subregion of the medial ganglionic eminence that appears intercalated between the pallidum and the preoptic area. The diagonal area $(\mathrm{Dg})$ roughly corresponds to the pMGE5 ventricular progenitor domain of Flames et al. (2007).

Somatostatin neurons were previously mapped immunocytochemically in rat and mouse embryos (e.g., Shiosaka 1992; García-López et al. 2008; Real et al. 2009). Compared to these earlier studies, our broader in situ hybridization analysis in different section planes and more embryonic stages provides much additional detail about their development, particularly because we explored in adjacent sections the relationships of Sst cells with the subpallial expression domains of several transcription factors $(G b x 2, D l x 5, L h x 7-8, N k x 2.1, N k x 5.1)$, as well as with transcripts of $S h h$, the gene coding the morphogen $\mathrm{SHH}$, significantly expressed in a large population of preoptic cells that invade the pallidum (Flandin et al. 2010). In agreement with Sousa et al. (2009), we detected the first telencephalic Sst cells at E10.5. In contrast, Shiosaka (1992) first illustrated such cells in E16.5 rat embryos (equivalent to E14.4 mouse embryos; Clancy et al. 2001). Similarly, Garcia-López et al. (2008) only reported data in E14.5 mice, while Real et al. (2009) saw scattered cells in the mouse Dg at E11.5. These are all immunocytochemical studies.

Authors who examined the origin of cortical Sst cells via transgenic and in vitro approaches generally concluded that these originate from a dorsal pallidal part of the MGE (see specific citations below), whereas García-López et al. (2008), Real et al. (2009) and Medina and Abellán (2012) associated the origin of $S s t$ cells that invade the amygdala to the anterior entopeduncular area or $M G E c v$ (i.e., the $\mathrm{Dg}$ ), in agreement with our present conclusions. In the following sections, we will comment on the development of telencephalic Sst cells, and discuss the conflicting views about the origin of these cells.

\section{Early development of $S s t$ cells}

In the present report, we mapped descriptively the progressive changes observable in the topography of $S s t$ cells in the developing mouse telencephalon. Given the existence of several experimental transgenic tracings demonstrating that such cells migrate tangentially from a restricted (single) subpallial source into other telencephalic domains (see citations below), we have parsimoniously interpreted the observed absolute changes in topography as evidence of tangential migration, starting at the apparent source (i.e., where the earliest cells are found). Our results revealed that the earliest $S s t$ cells identified at E10.5 are not uniformly distributed within the MGE, being restricted to a thin radial domain of the MGE mantle zone that appears intercalated between the pallidal and preoptic regions, as identified by differential markers. This is precisely the estimated position of the Dg (Puelles et al. 2013; Fig. 1b) and roughly correlates with the ventricular pMGE5 domain of Flames et al. (2007). Our correlative mappings of diverse subpallial markers at E10.5 and E11.5 showed that the early Sst population does not overlap either with the massive subpopulation of $G b x 2$ neurons that appears restricted to the pallidal area, nor with $N k x 5$.1-expressing preoptic neurons. We detected also no significant overlap between early $S s t$ cells and the sizeable stream of Shhpositive cells that migrates tangentially from the preoptic area into the pallidal mantle. Nevertheless, we systematically observed that the Dg ventricular zone itself shows patchy $S h h$ expression, a pattern that is distinct from the massive expression observed at the nearby preoptic POA1 ventricular zone; a small parallel contribution of $\mathrm{Dg}$ to the Shh-positive population in the subpallial mantle seems thus possible, though such cells would not overlap the Dg- 

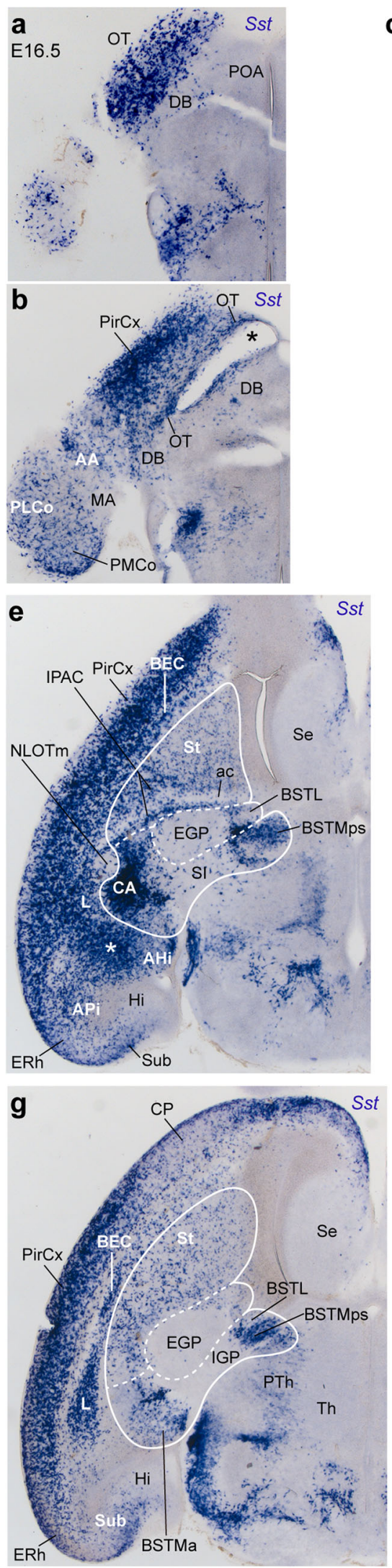

C
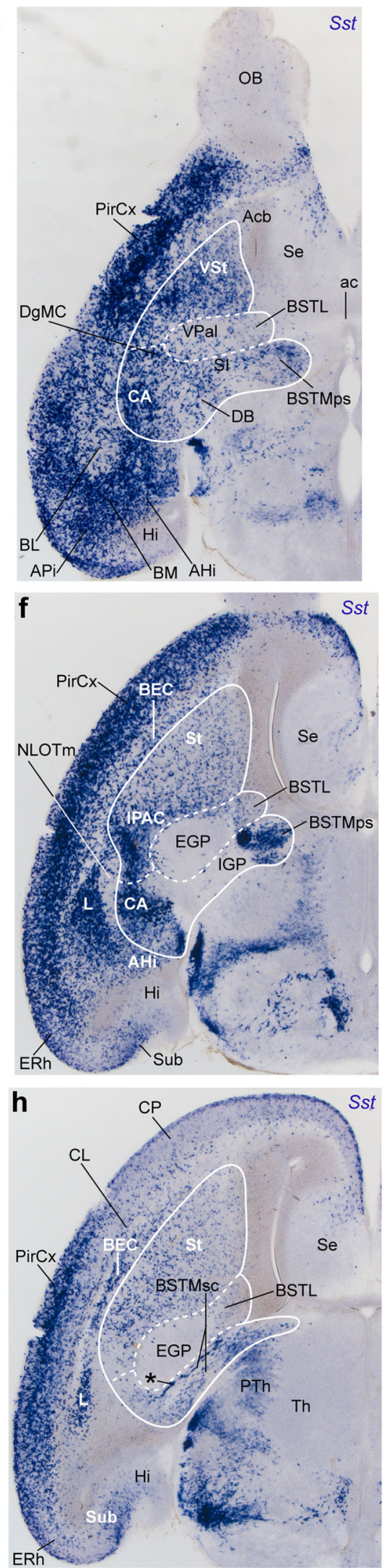

d

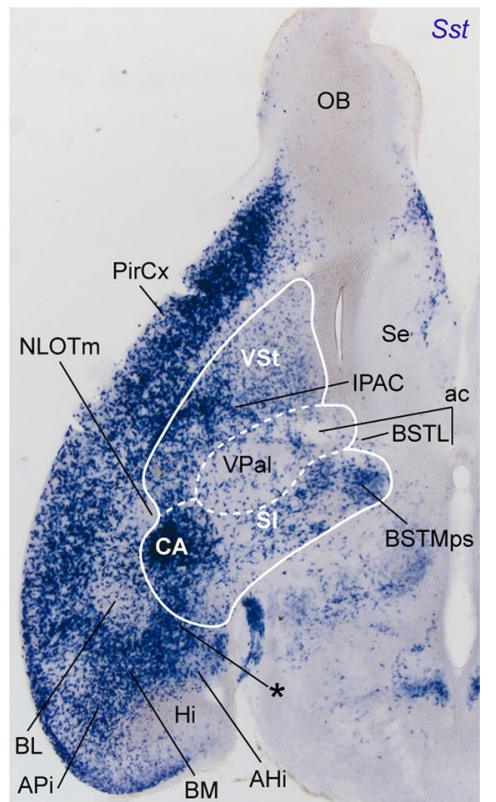


4Fig. 12 Selected horizontal sections through an E16.5 embryonic brain, showing the telencephalic distribution of Sst cells at this stage. The subpallial complex-St, Pal, Dg-is contoured by a continuous white line, and white dash lines separate these three domains. a, b The olfactory tuberculum (with many Sst cells) and the basalmost part of the amygdala are cut tangentially (the latter with labeling of the anterior amygdala, AA, and the posterolateral and posteromedial cortical nuclei, PLCo, PMCo). The diagonal band is partly seen (the asterisk marks an artefactual distortion). c, d Levels of section through the crossing of the anterior commissure (ac): intense labeling of the piriform cortex (prospective layer III) continues rostrally into the posterior part of the anterior olfactory area; few cells reach the olfactory bulb, and septal labeling is limited to medial portions (Se). The ventral striatal mantle is well populated by Sst cells (VSt), contrasting with the sparser population within the ventral pallidum (VPal). The diagonal domain is represented by the diagonal band (DB), the substantia innominata (SI), and the paraseptal BSTM nucleus (BSTMps). The subpallial amygdala displays the strongly labeled DgMC and CA derivatives, rostromedially to the relatively unlabeled BL nucleus, whereas the amygdalopiriform area (APi), $\mathrm{BM}$, and AHi amygdalar areas are strongly labeled. e, f The temporal fibers of the anterior commissure traverse the positive IPAC formation (interstitial nucleus of the posterior limb of the anterior commissure) at the back of the striatal mantle, which is limited laterally by a dense population at the bed nucleus of the external capsule, a ventral pallium derivative, jointly with the piirform cortex (BEC; PirCx); caudally, BEC seems continuous with the larger lateral amygdalar nucleus (L), which is also densely labeled. At the interface between $\mathrm{L}$ and CA there appears a round or elongated comet-shaped domain devoid of Sst cells that corresponds to the migrating primordium of the nucleus of the lateral olfactory tract (NLOTm). At the rostromedial end of the supracapsular BSTM arch there appears the Dg paraseptal BSTM derivative (BSTMps), which now shows several subdivisions. $\mathbf{g}, \mathbf{h}$ At these dorsal levels the $\mathrm{L}$ amygdalar nucleus diminishes in size, still showing continuity with the BEC, deep to the piriform cortex (PirCx) and next to the striatum (St). A linear claustral aggregate can be distinguished in $\mathbf{h}$ (CL). The amygdaloid and paraseptal ends of the BSTM arch meet over the internal capsule and the IGP, forming the supracapsular region (BSTMsc)

derived Sst population (possible salt-and-pepper pattern, separately also implied in the hypothesis that some cholinergic poulations are locally produced). The subpallial nature of the diagonal Sst cell population is corroborated by the shared expression of general subpallial markers such as Dlx5, Nkx2.1, and Lhx7/8.

Already at E10.5, it can be seen that the Dg radial stream of Sst cells is continuous superficially with an incipient subpial aggregate of similar cells spreading tangentially lateralwards; at this stage, these subpial cells partially cover marginally the pallidal mantle core occupied selectively by $G b x 2$ - and $S h h$-positive cells. A few of these subpial Sst elements even penetrate the striatal marginal stratum (identified as the subpallial mantle zone devoid of the $N k x 2.1$ pallidal marker). This superficial subpallial migration stream (abbreviated as SSpM) becomes much better developed at subsequent stages (E11.5-E14.5). It clearly represents the main pathway for the arrival of diagonal Sst cells at the striatum (complemented by outside-in radial invasion from the SSpM) and the pallium (first traversing subpially the prospective layer III stratum of the olfactory cortex primordium, which transiently lies at the brain surface at these early stages; Valverde and Santacana 1994); Sst cells then enter the marginal stratum of the insula and proceed into the isocortex in a gradiental pattern (the hippocampus and entrorhinal cortex seems to be invaded through an analogous caudal marginal stream across the amygdala). Our data indicate that the alternative intermediate and subventricular migration pathways so common for other subpallial migrating cortical interneurons (Anderson et al. 2001; Marín and Rubenstein 2003) are of minor importance in the case of the Sst cells. In contrast, a subventricular tangential migration route seems relevant for the invasion of the pallial amygdala, in addition to the SSpM (compare Wang et al. 2010; their Fig. 2).

Though the SSpM first crosses the pallidal marginal stratum at the locus of the prospective pallidal olfactory tuberculum (where a subpopulation persists at later stages), the pallidal mantle core remains practically devoid of $S s t$ cells at all stages, as is true as well for the mature globus pallidus and, partly, for the lateral BST formation (also pallidal in nature). This apparently reveals a persistent nonpermissive or repellent pallidal effect on the migrating $S s t$ cells; this phenomenon may explain as well the paucity of $S s t$ cells that migrate subventricularly into the striatum and the cortex, since they need to cross the pallidal territory. Moreover, no Sst cells were ever observed within the preoptic area; this result indicates a clearcut spatial orientation of the SSpM in the opposite palliopetal direction, possibly influenced by repellent signals spreading out of the preoptic area. It may be speculated whether SHH highly present in the preoptic and pallidal environments is repulsive for the Sst cell population (compare $\mathrm{Xu}$ et al. 2010).

\section{Ulterior migration and development of definitive Sst cell populations}

Independently of the $S s t$ cells that migrate tangentially away from the Dg via the SSpM and the deep and superficial amygdalar streams, the Dg histogenetic area also develops radial derivatives that mature locally; these appear thereafter clearly intercalated in the subpallial mantle between the Sst-negative pallidal and preoptic areas. This locus corresponds to a radial domain placed obliquely along the telencephalic stalk, which has received little embryological attention per se so far, but is known to exist since the nineties (the anterior entopeduncular area or AEP of Bulfone et al. 1993). This locus also shows 
characteristic structure in the adult. Previous morphologic analysis suggested that the Dg superficial stratum contains the bed nuclei of the diagonal band, extending obliquely (diagonally) from the amygdala to the medial septum. In its turn, the Dg intermediate stratum deep to the diagonal band forms the classic substantia innominata (which encompasses a major part of the cholinergic basal magnocellular nucleus of Meynert, but also contains other cell types). Finally, the corresponding Dg periventricular stratum contains what can be defined as the medial BST formation. The literature contains a clear idea of medial and lateral BST regions (e.g., in De Olmos et al. 1985), but confusingly also includes a diversity of classifications of individual BST nuclei into these main compartments, but we do not need to discuss these in detail here (compare, for instance, Ju and Swanson, 1989; Ju et al. 1989; Walter et al. 1991; De Olmos et al. 2004; Medina and Abellan 2012). Puelles et al. (2013) suggested, and we now corroborate, that the lateral BST formation (BSTL) is pallidal, whereas the medial BST (BSTM) is diagonal, as noted in terms of relative abundance of Sst neurons (see Walter et al. 1991 for the human brain) and differential expression of Isll (only in BSTL). The BSTM also includes some supra- and subcapsular elements classified by some authors within the medial extended amygdala (e.g., Medina and Abellan 2012). Note Medina and collaborators already interpreted part of the extended amygdala as a radial derivative of the Dg, identifying it as 'entopeduncular area' in Garcia-Lopez et al. (2008), or as 'caudoventral MGE' in Medina and Abellán (2012). The 'extended amygdala' concept, which is supported by the present results, essentially underlines the developmental sharedness of molecular, histogenetic, and other (e.g., hodologic) properties along the septo-amygdaloid complex of subpallial areas (Fig. 1a-c), as was realized by Heimer himself (personal communication to LP).

We cannot postulate from our material that all Dg derivatives express $S s t$, though we presently do not know of other selective markers of potential Dg mantle derivatives other than Chat, which identifies cholinergic neurons. At the most advanced developmental stage examined by us (E16.5) we saw rather sparse Sst cells at the level of the diagonal band nuclei, together with a rather dispersed Sst population within the innominate area deep to them; in contrast, there appears a well-developed population of large and small $S s t$ cells centered within the periventricular BSTM formation. Importantly, the latter population was found to lie just outside the pallidal domain expressing $N k x 2.1$ (or NKX2.1) from E13.5 onward (apparently due to down-regulation of initial $N k x 2.1$ expression at the $\mathrm{Dg}$ ), while it clearly lies within the Dlx5-expressing subpallial mantle (Figs. 9i, 1, 11 and S2); this observation was unexpected, since it is widely accepted in the field that the whole MGE (including Pal, Dg and POA) initially expresses $N k x 2.1$, and it was assumed that this state was permanent throughout the MGE. There exists as well a parallel supracapsular migratory stream of hypothalamic Otp-positive neurons that approach the medial amygdala (Wang and Lufkin 2000; Garcia-Moreno et al. 2010; Morales-Delgado et al. 2011). We checked whether this periventricular $O t p$ population coincides with the Sst one at the telencephalic stalk However, the Otp stream uses the thin pallial corridor that directly connects the alar hypothalamus with the pallial amygdala (topologically caudal to the subpallium as a whole), and therefore does not coincide with the Sst-positive BSTM; it demonstrably passes just outside the Dlx5-positive domain that contains the subpallial Sst BSTM elements (Figs. 9i, 1, 11b-f; S2ac).

The cholinergic neurons of the diagonal band and the substantia innominata (basal magnocellular nucleus) probably represent a significant part of the Sst-negative intermediate and superficial Dg mantle (as suggested by material shown at the Allen Adult Mouse and Developing Mouse Brain Atlases, or García-López et al. 2008). Given the recent unexpected notion that many of these cells (but not all) originate distantly in the ventral pallium and belong to a Tbrl-expressing lineage that invades the Dg (Pombero et al. 2011), it is open to discussion how far the few Sst cells present in these superficial Dg areas may represent the local intrinsic diagonal population, in contrast with local and immigrated cholinergic cells. On the other hand, Zhao et al. (2003) reported a dependency of the subpallial cholinergic cell type on $L h x 8$ gene expression, a pattern that is restricted to the Pal and Dg regions of the MGE (as was corroborated by present data; Fig. S1). These authors just assumed a local subpallial origin of the cholinergic cell type, without commenting on the potential role of diverse MGE progenitor subareas in their production, an issue subsequently underlined by Flames et al. (2007). The demonstrated requirement of $L h x 8$ signal in the MGE for the local differentiation of cholinergic neurons (though these potentially may originate elsewhere) may bespeak of an indirect non-cell-autonomous effect due to local MGE mantle conditions controlled by Lhx8. According to the results of Pombero et al. (2011) such an effect may act upon cells previously produced in the pallium under control of $\mathrm{Tbrl}$ and secondarily migrated tangentially into the diagonal part of MGE. If this were not so, we would expect cholinergic cells differentiating massively everywhere the Lhx8 signal appears in the subpallium, that is, in the whole $\mathrm{Pal}$ and Dg domains, which is not the case. This hypothesis accordingly may conciliate the data of Zhao et al. (2003) and Pombero et al. (2011). The fact that most basal cholinergic neurons finally populate the $\mathrm{Dg}$ area (diagonal band nuclei and basal magnocellular population within the 
substantia innominata) suggests a differential local microenvironment (more specific than is allowed by the full domain of $L h x 8$ expression), and further supports the notion that the $\mathrm{Dg}$ is a molecularly distinct from the Pal (Flames et al. 2007), as is also indicated by the restricted local downregulation of $N k x 2.1$ and the selective production of Sst cells. Interestingly, Zhao et al. (2003) observed that Sst cells were not affected by the null mutation of Lhx8.

The supracapsular BSTM arch that forms at amygdaloid, central, and paraseptal periventricular regions of the $\mathrm{Dg}$ area (terminology of Puelles et al. 2013; Fig. 1b) appears well populated by Sst cells from E14.5 onward; it is first visible as an emergent aggregate of Sst cells at E13.5 (note Bayer, 1987 found in the rat birthdates between E15 and E17 for what Puelles et al. 2013 call the 'paraseptal BSTM portion'; these rat stages correspond to E13.6-14.8 in the mouse; Clancy et al. 2001). The rather compact Sst-positive BSTM population stands out from the largely Sstnegative pallidal BSTL formation, which overlies the globus pallidus (incidentally, we think that our observation that external and internal globus pallidus parts can be distinguished according to their topography relative to the Sst-positive BSTM arch-the latter covers directly the IGP (see Fig. 11) - may be aclaratory with respect to the traditional concept that the IGP originates in the hypothalamus). The BSTL locus only transiently shows a few Sst cells that migrate tangentially through the pallidal subventricular stratum into the striatum (or the pallium). The BSTM supracapsular arch extends across the paraseptal Dg area (which neighbours the crossing fibers of the anterior commissure) into the diagonal part of the subpallial medial septum, where individual parts of the BSTM formation have been recognized in adult rodents (these are often described as 'anterior BST' in the relevant literature). The amygdaloid BST nucleus is wholly or in part a caudal prolongation of the supracapsular BSTM into the amygdaloid part of the subpallium (Puelles et al. 2013; Fig. S4).

The potential existence of intrinsic amygdaloid $\mathrm{Dg}$ derivatives-and Sst cells-that are not tangentially migrated from outside the amygdala proper was apparently never considered previously. The existence of an amygdaloid diagonal subpallial subdomain first appeared defined in the Allen Developing Mouse Brain Atlas (online since 2009; see also Puelles et al. 2013). Our present results strongly suggest that at least a significant aggregate of Sst neurons later found within the lateral subregion of the central amygdaloid nucleus, which is widely accepted as a part of the subpallial amygdala, derives primarily via radial migration from the amygdaloid pole of the $\mathrm{Dg}$ progenitor area (CeL in Fig. S3A, B; Ce in Fig. S4; the medial and capsular parts of the central amygdalar nucleus may be pallidal or striatal in origin). Bupesh et al. (2011a) reported a tangentially migrated contribution to $\mathrm{Ce}$ of more rostral parts of the Dg domain (their MGEcv area), but these experiments did not include labeling of the amygdalar end of the Dg complex, and were generally performed at E14.5, which we believe is too late to detect the radial migration we deduce forms the CeL; we start to observe this primordium already at E12.5. The presence of appropriately oriented radial glia fibres across the $\mathrm{Ce}$ is shown in our Fig. S4. The diagonal magnocellular nucleus (DgMC; known in the literature as 'preoptic magnocellular nucleus') seems also an intermediate stratum derivative of the amygdaloid Dg subdomain. In our identification of this cell group in a position lateral to the locus of the horizontal diagonal band nucleus we followed standard rodent atlas advice (Watson and Paxinos 2010; note Paxinos and Franklin 2013 identified this nucleus as 'lateral nucleus of the diagonal band', considering as we do that it is not preoptic in nature). As is shown in several of our Figures (Figs. 1b, 8, 10p, q, 12), our data indicate that the diagonal amygdala contacts directly the striatal amygdala behind the caudal end of the pallidal amygdala (the striatal amygdala is mainly a caudal part of the dorsal striatum, e.g., according to ER81 and Six3 expression). This arrangement had not been disclosed so far.

The lateral central amygdaloid subnucleus (CeL) is a compactly Sst-positive population identifiable as an incipient radial migratory stream at the amygdaloid Dg subarea already at E12.5 (asterisk in Fig. 7f, g). This mass later appears systematically intercalated between the striatopallidal complex and rostral parts of the pallial amygdala (L/BL primordia); it characteristically preconfigures the future CeL nucleus from E13.5 onward (Figs. 8e-g, 9a, $10 \mathrm{~g}-\mathrm{j}, \mathrm{p}, 11 \mathrm{a}, 12 \mathrm{c}-\mathrm{f})$. We did not find any reference to this particular subpallial primordium in the embryologic literature, nor had we been aware ourselves of its existence previously. Representative sections shown in our Figs. 10 and 12 illustrate the histogenetic and genoarchitectonic continuity of the CeL primordium with the supracapsular BSTM arch, providing a novel insight into the development of this area. In contrast, Medina and Abellán (2012) recently interpreted that $S s t$ cells reach the central amygdala via tangential migration from the 'medioventral MGE' (equivalent in their schemata to our paraseptal and/or central Dg; see comments above about similar conclusions of Bupesh et al. 2011a). The amygdaloid end of the BSTM component of $\mathrm{Dg}$ also apparently builds separately the well-known amygdaloid BST nucleus (BSTA). We thus believe this would represent likewise a radially migrated Dg-derived entity, rather than a result of tangential migration.

On the other hand, numerous tangentially migrating Sst cells invade the pallial amygdala, either via the subpial SSpM, or via a specific, well-developed, amygdaloid 
subventricular migratory pathway. We could not assess whether these cells originate specifically from paraseptal, central, or amygdaloid parts of the Dg area, or come from all of them. A feature suggesting a general Dg origin is that the earliest cells invading the pallial amygdala via the SSpM were observed at E11.5 (Fig. 5ae, af), and this pathway was still very distinct at E12.5 (superficial arrow; Fig. 7e, f). The subventricular subpallial migratory stream (SvSpM) that likewise targets the pallial amygdala appeared at E12.5 in a relatively more dorsal (perhaps more origin-selective) position (SvSpM; deep arrow; Fig. $7 \mathrm{~g}-\mathrm{k})$. It is possible that some of these deep SvSpM cells reach the overlying cerebral cortex (entorhinal and hippocampal areas, and perhaps even occipitotemporal areas) via a transamygdalar route. Both streams are less distinct at E13.5 and subsequent stages; this later period is characterized by a substantial invasion by $S s t$ cells of the lateral (L), basomedial (BM) and amygdalohippocampal (AHi) amygdaloid nuclear primordia. There is the apparent exception of the basolateral, posteromedial cortical, and medial amygdaloid nuclei, which stand out as loci with few Sst neurons (BL, PMCo, MePV, MA; Figs. 10, 11, 12).

At E16.5, the L primordium appears densely populated and even delineated by $S s t$ cells (Fig. 12f-h). Horizontal sections show that the rostral tip of $\mathrm{L}$ roughly coincides with the locus where the fibers of the anterior commissure reach the amygdala. Similar numbers of Sst cells label the IPAC nucleus, a part of the extended amygdala (interstitial nucleus of the posterior limb of the anterior commissure; Fig. 12d-f). More rostrally, along the line where the anterior limb of the anterior commissure enters the external capsule (lateral to the striatum) a distinct laminar population of Sst cells is visible which appears in a similar place as the $\mathrm{L}$ nucleus, but lies rostral to it, and is much thinner. We think that it corresponds to the primordium of the bed nucleus of the external capsule (BEC), a formation derived from the ventral pallium that was recently distinguished at this locus (Puelles 2014); this primordium probably also corresponds to the supposedly transient 'reservoir' of Bayer and Altman (1991). The amygdalar L nucleus is held to be a ventropallial derivative on genoarchitectonic grounds, whereas the BL was ascribed to the lateral pallium (Medina et al. 2004). There accordingly appears to exist a preferential invasion of ventropallial cell masses by the tangentially migrated Sst cells as they pass beyond the pallio-subpallial boundary (this includes the large population invading layer III of the piriform cortex, which also is an integral part of the ventral pallium (Puelles 2014). At postnatal stages, further development of the pallial amygdaloid nuclei and their neuropil leads to substantial dilution or decrease by cell death of the contained population of Sst cells, though $\mathrm{L}$ still retains in the adult more $S$ st cells than the BL nucleus (compare L and BL in Fig. S3b).
Our analysis of the invasion of the isocortical plate by Sst cells was largely centered on the chronology of the arrival of these cells to the different areas. By E16.5 the whole cortex was covered superficially by $S s t$ cells. As mentioned above, we believe that the main pathway for this tangential migration is the SSpM. Following Puelles (2014), we interprete that marginal Sst cells observed over the cortical plate up to E14.5 result subsequently distributed to the subgranular cortical layers (since the cortical plate at E14.5 is largely formed by the prospective layer 5 and layer 6 pyramids). As was previously reported, the major adult population of cortical Sst interneurons has a subgranular topography (see Fig. S3b). Presumably, labeled embryonic cells occupying a similar marginal position at E15.5 and E16.5 will be distributed to the sparser population later found in the supragranular layers.

\section{Telencephalic subpallial domains and the origin of Sst cells}

A subpallial origin of most inhibitory cortical interneurons in the mouse is supported by the fact that practically all cortical interneurons in mice derive from the Dlx5/6-expressing subpallial lineage (Stuhmer et al. 2002), and such cells are not part of the massive complementary pallial Emxl-expressing lineage (Iwasato et al. 2000; Gorski et al. 2002). Whereas some pallial explants do give rise to some cells producing GABA in vitro (Götz et al. 1995; He et al. 2001; Bellion et al. 2003; Nery et al. 2003), it is so far unclear whether these results are extrapolable to the in vivo situation (they may result alternatively from differences in genomic regulation created by in vitro conditions, or from a potential initial content of migrating cells of subpallial origin capable of spontaneous or stimulated proliferation in the explanted tissue). In humans, Letinic et al. (2002) reported that more than half the population of cortical inhibitory interneurons derives from mitoses occurring within the pallial subventricular zone (but see Ma et al. 2013).

Our results corroborate previous data suggesting that SST-positive cells reaching the amygdala derive from the Dg domain (old AEP; García-López et al. 2008; Real et al. 2009; Bupesh et al. 2011a, b). We provide here a more detailed description of the relationship of the Dg area with Sst neuron production and migration, which leads us to suggest a Dg origin for most cells expressing Sst in the telencephalon. This seems partly contradictory with some earlier experimental results, which suggested that the major origin of such cells is the pMGE1 sector of the pallidum, which clearly does not form part of the Dg (Fig. 1c). We think the analysis of this issue (and other analogous issues pertinent to the subpallium) improves by translating the diverse contributions into the comprehensive conceptual 
framework developed for the subpallium in the Allen Developing Mouse Brain Atlas (reference atlases, online since 2009; www.developingmouse.brain-map.org); see also Puelles et al. (2013). This model allows striatal, pallidal, diagonal, and preoptic alternative origins of the Sst cells to be visualized (Fig. 1a, b), and diverse septal, paraseptal, central and amygdaloid sectors along these domains can be located rather precisely with reference to the septo-amygdaloid axis, as well as periventricular, intermediate and superficial strata with regard to the radial dimension.

\section{Striatal origins}

A striatal origin of Sst cells can be dismissed straightforwardly, because the cerebral cortex of $N k \times 2.1^{-1-}$ mutants, which essentially lose all MGE progenitor fates, and develop a larger striatum (Pal, Dg, and POA are repatterned as striatum-like domains; Sussel et al. 1999), contains practically no interneurons expressing Sst at E18.5 (Anderson et al. 2001). Additionally, primary cultures testing postnatal development of interneuron subtypes in the $N k x 2.1^{-1-}$ mutant cortex showed absence of somatostatin and parvalbumin interneurons (Xu et al. 2004).

\section{Pallidal versus diagonal origins}

Transgenic mice lines expressing GFP or LacZ reporters in the Nkx2.1Cre-labelled lineage were expected to show colabeling of all SST-positive cortical interneurons. Surprisingly, many parvalbumin- and SST-positive interneurons did not show the reporter, particularly in superficial cortical layers of $N k x 2.1 C r e: Z / E G$ and $N k x 2.1 C r e: R 26 R-L a c Z$ mice (Xu et al. 2008). Our observation that $N k x 2.1$ expression disappears at the $\mathrm{Dg}$ after E13.5-a period when many supragranular interneurons are produced-may be relevant to explain these data. Early Dg-derived cells might be represented instead among the reporter-colabeled infragranular population (see Sousa et al. 2009). Xu et al. (2008) conjectured that the subpopulation of SST cortical interneurons that was not colabeled in their transgenic mice might derive from the dorsal portion of the MGE (corresponding to the pMGE1 domain; Fig. 1c), on the rationale that Nkx2.1Cre activity was practically absent in the pMGE1 domain, as opposed to distinct $N k x 6.2$ expression. It was accordingly suggested that SST cells lacking $\beta$-gal reaction derive from pMGE1, whereas those that coexpress the reporter would derive from a different domain (Xu et al. 2008). This conclusion seemed consistent with earlier in utero fate-mapping studies $(\mathrm{Xu}$ et al. 2004; Butt et al. 2005). In any case, these data are also consistent with an origin of both early and late SST neurons within the Dg area (pMGE5), due to the observed local downregulation of $N k x 2.1$ expression there.
After initial experimental reports showed that the MGE gives rise to PV and SST cortical interneurons (Xu et al. 2004; Butt et al. 2005; Ghanem et al. 2007), Flames et al. (2007) performed in utero transplantation of GFP-expressing MGE cells into isochronic host embryos at E13.5. They found that around $30 \%$ of the GFP-positive cells coexpressed SST, as opposed to roughly $50 \%$ of cells coexpressing PV. In the same report, a small cube of GFPpositive tissue obtained from the dorsal pMGE1 subdomain at E13.5 (the pallidal domain closest to the striatum; Fig. 1c) was dissociated, and the cells were grafted into the MGE of an isochronic host embryo. The distribution of GFP-expressing cells in the host mice at P14 revealed that over $60 \%$ of transplanted cells were SST positive, against $7 \%$ labeling obtained with similar grafts of pMGE4 cells; no experiment was performed with cells from our Dg, which corresponds to pMGE5 (Flames et al. 2007; compare Fig. 1c). The dorsal Pal domain was accordingly proposed as the main origin of the $\mathrm{SST}^{+} / \mathrm{CR}^{+}$Martinotti cells, and this conclusion was corroborated by other authors (Flames et al. 2007; Fogarty et al. 2007; Wonders et al. 2008; see below). The concern raised by these apparently strong data, is that E13.5 may be a rather late stage for detecting the origin of Sst cells, since a substantial number of the corticopetal Dg-originated elements have probably slipped beyond the MGE at that stage; moreover, many migrating Dg elements must be present at the transient SSpM stream found crossing the pallidal marginal stratum at E13.5. Accordingly, the cells dissociated at the pMGE1 locus at E13.5 may have included a significant number of $\mathrm{Dg}$ derived Sst neurons. The conclusion of Flames et al. (2007) as regards the pMGE1 origin of Sst cells thus appears weaker than expected, pending further experimental tests with younger pMGE1 cells (e.g., taken at E10.5), and including comparisons with the diagonal pMGE5 area. Nevertheless, though we saw little histologic evidence of a pMGE1 origin of Sst cells, our results do not allow us to exclude categorically that some $S s t$ cells may arise at this pallidal subarea.

Wonders et al. (2008) dissociated dorsal and ventral GFP-positive Pal tissue at E13.5 and injected the cells into newborn cortex, checking their differentiation into SST versus parvalbumin (PV) cells. They concluded that the dorsal Pal was mainly implicated in the production of SST cortical interneurons (63\% SST versus $30 \% \mathrm{PV}$ ), whereas relatively more interneurons expressing parvalbumin apparently originated at the ventral MGE (31\% SST versus $59 \% \mathrm{PV})$. The location given for the ventral MGE in their Figure 2A is compatible with the topography of the Dg (the depicted explants most probably contained a deep part of the mantle zone, in our opinion). In order to eliminate potential passing cells, Wonders et al. (2008) explanted again dorsal Pal cells at E12.5 $1 \mathrm{~h}$ after injecting 
BrdU to the dams and cultured them in vitro for 10 days. The rationale was to obtain in vitro-differentiated SST neurons double-labelled for GFP and BrdU, that is, derived exclusively from dorsal $\mathrm{Pal}$ progenitors They obtained over $60 \%$ double-labeled SST cells for dorsal tissue versus $15 \%$ for ventral tissue (their Fig. 3F). A concern that applies to these data is whether differentiation of dissociated immature cells injected directly into the newborn cortex, or in vitro differentiation of BrdU-labelled progenitors, reproduces faithfully enough the in vivo conditions for the relevant phenotypic decisions. Moreover, the first results may be contaminated by migrating Dg cells passing next to pMGE1 (as in Flames et al. 2007), and the in vitro results may result from re-specification of the explanted dorsal Pal progenitors (e.g., by downregulation of their $N k x 2.1$ expression). Appropriate tests should be designed to check these possibilities. In any case, taken at face value, these results suggest that SST cortical interneurons are produced both at the dorsal and ventral $\mathrm{Pal}$, that is at the pMGE1 and pMGE5 domains, thus providing partial support for our results. This suggests that our negative data about Sst cells originating within the Pal, or their absence inside the early Pal mantle layer, might be caused by late transcription of the Sst gene in the pMGE1derived elements.

The homeodomain transcription factor $N k x 6.2$ is expressed at the border between striatal and pallidal domains. It is specifically observed in a small subset of neural progenitors localized across the striatal progenitor domain pLGE4 and the pallidal progenitor domain pMGE1 (Stenman et al. 2003; Flames et al. 2007; Fogarty et al. 2007; Sousa et al. 2009). Genetic inducible fate-mapping using $N k x 6.2 \mathrm{CreER} /+$ mice showed EGFP-colabeling in SST-positive cortical interneurons (Sousa et al. 2009). Interestingly, the $N k x 6.2-C r e / R 26 R-G F P$ transgenic mice line used by Fogarty et al. (2007) also showed GFP expression in scattered neuroepithelial cells located more ventrally in the caudal ventral MGE (the Dg area), suggesting that the results of Sousa et al. (2009) are not inconsistent with a double origin of SST cells. This work concluded that SST+/CR- cells generated at E10.5 selectively target deep cortical layers, whereas SST+/CR+ cells are generated at E12.5 and invade superficial cortical layers. The authors did not mention whether cells from this lineage differentiate within subpallial formations after radial migration.

Fogarty et al. (2007) concluded that only small subsets of the interneurons produced in their $N k x 6.2-C r e / R 26 R$ GFP transgenic mice line coexpress GFP and the studied interneuron markers (including SST; their Figure 3A-D). This generates the possibility that most SST cells are derived from other pallidal domains, including the $\mathrm{Dg}$. When Nkx6.2-Cre/Nkx2.1-Cre/R26R-GFP double transgenic mice (lineage tracing targeting all MGE and POA derivatives) were studied, the majority of SST + cells (70-80 \% of cortical motor and somatosensory SST cells) were marked with GFP. Since $N k x 2.1$ Cre activity is practically absent at the pMGE1 domain (Fogarty et al. 2007; $\mathrm{Xu}$ et al. 2008). This result suggests that most SST-positive cells are generated in the alternative source, the "caudal ventral MGE'. i.e., the Dg (Fogarty et al. 2007). Approximately $35 \%$ of $N k x 2.1 C r e-G F P$-expressing cells were SST positive and they represent around $70-80 \%$ of this type of interneuron in the motor and somatosensory cortex (Fogarty et al. 2007).

Poor recombination of R26R-GFP with Lhx6-Cre was also observed at the pMGE1 (Fogarty et al. 2007). Using both Nkx2.1Cre:R26R-GFP and Lhx6Cre:R26R-YFP, these authors thus essentially identified the origin of most SST interneurons as corresponding to the 'caudal ventral MGE', which represents the Dg domain. Lhx6Cre:R26R-YFP transgenic mice displayed in the motor and somatosensory cortex $100 \%$ of SST-positive cells labeled with YFP, as well as nearly $100 \%$ of the PV+ and CB+ interneurons. A high degree of SST and Lhx6 coexpression was also observed using $L h x \sigma^{+/ \text {LacZins }}$ mice, and Sst-expressing cells were drastically reduced (around 93\%) in Lhx $6^{-1-}$ mutants (Liodis et al. 2007, Zhao et al. 2003). These experimental approaches suggest strongly that the "caudal ventral MGE' (Dg) domain contains the main SST-cell source; early activity of the genes $N k x 2.1$ and $L h x 6$ seems required to generate SST-positive interneurons (Sussel et al. 1999; Liodis et al. 2007).

Carney et al. (2010) analyzed the lineage origin of medial amygdala components, immunolabeling SST cells in combination with an anti- $\beta$-galactosidase ( $\beta$-gal) antibody to visualize nuclear staining in recombined cells from Nkx2.1 ${ }^{\text {Cre }}, \mathrm{Shh}^{\mathrm{Cre}}$ and $\mathrm{Glil}^{\mathrm{CreER(T2)}}: \mathrm{Tau}^{\text {mGFP }}$ brains. This analysis revealed that 10 and $23 \%$ of $N k \times 2.1^{\text {Cre }}:$ Tau $^{\text {mGFP }}$ and 20 and $24 \%$ of Glil $^{\text {CreER(T2) }}:$ Tau $^{\text {mGFP }}$ recombined cells coexpressed SST at the medial posterodorsal and medial posteroventral nuclei (MePD, MePV) of the amygdala, respectively. This proportion was reduced to 2 and $3 \%$ of cells coexpressing SST and $S_{h}{ }^{C r e}:$ Tau $^{m G F P}$ in the MePD and MePV, respectively (Carney et al. 2010). These results led to the conclusion that SST cells that populate the medial amygdala derive from the "caudal ventral MGE', that is, the Dg.

Flandin et al. (2010) used Shh-Cre mice and a floxed $N k x 2.1$ allele to selectively knock-out $N k x 2.1$ function from the $S h h$-expressing subpallium (POA1 ventricular zone and Shh-positive migrated cells in the pallidal mantle; eventually, also some ventricular-cell patches in Dg). They observed that the globus pallidus cell population was substantially eliminated, whereas most cortical and striatal interneurons were generated, excepting the 
striatal cholinergic neurons. In their Discussion, the authors deduce that pMGE1-3 (perhaps also pMGE4) generate most parvalbumin + and somatostatin + neo-cortical and hippocampal interneurons, because these domains do not express Shh. According to our interpretation, the Sst cells originated from the $\mathrm{Dg}$ ventricular domain - the pMGE5 area-may have partly escaped the targetted knock-out, since Shh expression only appears there in a patchy fashion (Figs. 4, 5, 6, S1; note Flandin et al. 2010 themselves observed reduced integration of ShhCre activity in the Dg domain in ROSA;Shh ${ }^{\mathrm{Cre} /+}$ mice). A $40 \%$ of reduction in cortical SST + cells was observed in the ShhCre-Nkx2.1-floxed mutant (Flandin et al. 2010), consistently with the possibility that this represents the product of a Dg progenitor fraction (40\%) that expresses $S h h$, whereas the remaining SST+ elements may be originated by the $S h h$-negative Dg progenitors, and are thus unaffected. On the other hand, a strong knock-out effect probably occurred at the septal Dg/POA sectors, where $S h h$ signal is intense in the ventricular zone (Figs. 4, 5, 6, S1); this is the place where striatal cholinergic neurons are perhaps produced (García-López et al. 2008; Hoch et al. 2015). The reported high activation of ShhCre in several pallidal domains is difficult to understand, because $S h h$ is not expressed at all in the pallidal ventricular zone (present results); the missing or reduced globus pallidus phenotype may be due to a strong knock-out effect at the POA1 area, whose ventricular zone expresses strongly $S h h$ and produces $S h h$-positive neurons that migrate into the pallidal mantle. These migrated cells may release $\mathrm{SHH}$, which might be required for the normal development of the globus pallidus (e.g., maintenance of Nkx2.1 activity).

Most of these in vivo and in vitro fate-mapping and transgenic mice studies thus suggest a principal 'ventral MGE' main source of Sst cells, consistent with our Dg area, highlighted in our in situ analysis as the main origin of this interneuronal type. Our study emphasizes the supra- and subcapsular histogenetic and topographic unity of the Dg domain along the septoamygdaloid axis, where various radially migrated derivatives are established (diagonal complex/medial septum, BSTM, amygdaloid BST and CeL nuclei), and from where laterally oriented tangential migrations proceed into the striatum and the whole pallium (sidestepping via the SSpM the apparently nonpermissive or repelling pallidum). Several of the cited studies proposed that GABAergic interneurons from the MGE (including SST cells) first invade the pallium around E12.5 (e.g., Corbin et al. 2001; Marin and Rubenstein 2003); our sequential analysis suggests that this process already starts at E10.5 out of the Dg domain, and pioneering cells already reach the cortex around E12.5.

\section{Preoptic origins}

The POA region forms the ventromedial Nkx2.1-positive component of the MGE. It shows three dorsoventrally superposed progenitor domains, identified as POA1 (next to Dg), POA2 (next to the terminal lamina) and $\mathrm{POH}$ (preopto-hypothalamic transition area, limiting with the paraventricular alar hypothalamus) (Flames et al. 2007; Bardet et al. 2010; Medina and Abellan 2012; Puelles et al. 2012, 2013). Lineage-tracing experiments using intra-utero electroporation of a Nkx5.1Cre:R26R-YFP construct demonstrated labeled preoptic GABAergic cells that migrate tangentially into the cortex, septum, striatum, and amygdala (Gelman et al. 2009). These cells frequently coexpressed NPY, but never SST. Moreover, they derive from a $N k x 2.1+/ L h x 6$ - lineage, which confirms their POA identity (Gelman et al. 2009). Preoptic Nkx5.1 signal present in postmitotic cells was initially held to be a general POA marker (Wang et al. 2000; Gelman et al. 2009), but Gelman et al. (2011) later acknowledged that these cells originate specifically at the Shh-positive POA1 domain, as is corroborated by our present data (see Fig. 6); indeed, we found that this restricted origin includes the median or acroterminal part of pPOA1, which encompasses the terminal lamina (Puelles et al. 2012). Gelman et al. (2011) stated that the Nkx5.1-positive elements that migrate out of the pPOA1 area rapidly lose this expression. Comparison of $N k x 5.1$ expression with $S h h$ expression in the subpallial mantle indicated that preoptic Shh-expressing cells are likewise produced selectively within the pPOA1 area, from where they selectively migrate tangentially into the pallidum (after crossing the Dg domain; note we never saw them entering the striatal mantle). In the chick and mouse, the pPOA2 area expresses $N k x 2.1$, but not $S h$ (Bardet et al. 2010; Flandin et al. 2010). Gelman et al. (2011) investigated a subarea of pPOA2 that expresses $D b x l$, from where a separate population of derived GABAergic cells migrate into the cortex, invading predominantly its deep layers. Phenotypic analysis of these cells at P14 suggested that nearly $50 \%$ of the cortical preoptic-derived $D b x l$ cells contain PV (the authors comment that the proportion may be larger, though, since this marker is a late differentiating one, and many of the observed GABAergic Dbxl-derived cells did not express any peptidic markers) and approximately $25 \%$ contained SST. They deduced that both PV and SST cells are produced at the pPOA2 area. This observation is contradictory with our observations, because we never saw $S s t$ cells arising within the preoptic area. We would thus predict that the SST elements observed by Gelman et al. (2011) most probably originated likewise in the Dg area. Primary evidence that can be adduced in favor of this interpretation is the expression 
of Dbxl mapped at E11.5 in the Allen Developing Mouse Brain Atlas (http://www.developingmouse.brain-map.org); the available sagittal and coronal sections both illustrate that at this stage the expression domain of $D b x l$ extends more importantly across Dg than across the preoptic area. Part of the Dbxl-derived cells may be thus diagonal in character, rather than preoptic. Gelman et al. (2011) further acknowledged that permanent tracing of Dbxlderived cells in Dbx $1^{\mathrm{Cre}}$; ROSA26 ${ }^{\mathrm{YFP}}$ embryos generated 'a few clones of YFP-expressing cells... in the MGE'. The authors checked the relationship of Dbxl-derived cells with Lhx6-derived cells (Lhx6 is a general MGE marker excluded from the POA; Liodis et al. 2007); the results indicated that some $36 \%$ of the cortical Dbxlderived cells co-express $\operatorname{Lh} x 6$, confirming that their origin can not be purely preoptic. On the other hand, Fogarty et al. (2007) concluded that $100 \%$ of cortical SST cells express Lhx6. This suggests that the $25 \%$ of SST neurons counted by Gelman et al. (2011) among the cortical Dbxl progeny may originate as we suggest within the $D b x 1+l$ Lhx6+ Dg sector of the MGE.

To summarize, in our opinion the published experimental evidence on lineage-tracing of SST-positive cells seems somewhat vague and inconclusive about their precise origin within the MGE, partly due to 1) confusion in the denomination and/or tracing of the relevant progenitor areas, 2) experimental study mainly of suboptimal stages (e.g., E13.5) which allow already migrated, or migrating neurons, to be ascribed to a wrong source, and 3) insufficient use (or dearth) of efficient molecular delimitation criteria for the Pal, Dg and POA subpallial domains and their diverse septoamygdaloid sectors. The present descriptive analysis cannot correct all these problems, but provides a solid basis of data that must be made consistent with any experimental analysis, as long as the existence of invisible Sst cells is not demonstrated beyond any doubt. They also illuminate in a novel way the development of the BSTM, CeL, and globus pallidus formations, as well as the intraamygdaloid relationships of the main subpallial domains.

Acknowledgments This work was funded by the Spanish Ministry of Science and Innovation grant BFU2008-04156, and SENECA Foundation contract 0458/GERM/06-10891 to L.P.; and the Local Government of Castilla-La Mancha grant PII1I09-0065-8194 to C.D. Infrastructure support provided by the University of Murcia and Castilla-La Mancha is also acknowledged.

Open Access This article is distributed under the terms of the Creative Commons Attribution 4.0 International License (http://crea tivecommons.org/licenses/by/4.0/), which permits unrestricted use, distribution, and reproduction in any medium, provided you give appropriate credit to the original author(s) and the source, provide a link to the Creative Commons license, and indicate if changes were made.

\section{References}

Anderson SA, Marín O, Horn C, Jennings K, Rubenstein JL (2001) Distinct cortical migrations from the medial and lateral ganglionic eminences. Development 128:353-363

Bardet SM, Cobos I, Puelles E, Martinez-de-la-Torre M, Puelles L (2006) Chicken lateral septal organ and other circumventricular organs form in a striatal subdomain abutting the molecular striatopallidal border. J Comp Neurol 499:745-767

Bardet SM, Ferran JL, Sanchez-Arrones L, Puelles L (2010) Ontogenetic expression of sonic hedgehog in the chicken subpallium. Front Neuroanat 21:1-16. doi:10.3389/fnana.2010. 00028

Bayer SA (1987) Neurogenetic and morphogenetic heterogeneity in the bed nucleus of the stria terminalis. J Comp Neurol. 265:47-64

Bayer SA, Altman J (1991) Development of the endopiriform nucleus and the claustrum in the rat brain. Neuroscience 45:391-412

Bellion A, Wassef M, Métin C (2003) Early differences in axonal outgrowth, cell migration and GABAergic differentiation properties between the dorsal and lateral cortex. Cereb Cortex 13:203-214

Brazeau P, Vale W, Burgus R, Ling N, Butcher M, Rivier J, Guillemin R (1973) Hypothalamic polypeptide that inhibits the secretion of immunoreactive pituitary growth hormone. Science 179:77-79

Bulfone A, Puelles L, Porteus MH, Frohman MA, Martin GR, Rubenstein JL (1993) Spatially restricted expression of Dlx-1, Dlx-2 (Tes-1), Gbx-2, and Wnt-3 in the embryonic day 12.5 mouse forebrain defines potential transverse and longitudinal segmental boundaries. J Neurosci 13:3155-3172

Bulfone A, Smiga SM, Shimamura K, Peterson A, Puelles L, Rubenstein JL (1995) T-brain-1: a homolog of Brachyury whose expression defines molecularly distinct domains within the cerebral cortex. Neuron 15:63-78

Bupesh M, Legaz I, Abellán A, Medina L (2011a) Multiple telencephalic and extratelencephalic embryonic domains contribute neurons to the medial extended amygdala. J Comp Neurol 519:1505-1525. doi:10.1002/cne.22581

Bupesh M, Abellán A, Medina L (2011b) Genetic and experimental evidence supports the continuum of the central extended amygdala and a mutiple embryonic origin of its principal neurons. J Comp Neurol 519:3507-3531. doi:10.1002/cne.22719

Butt SJ, Fuccillo M, Nery S, Noctor S, Kriegstein A, Corbin JG, Fishell G (2005) The temporal and spatial origins of cortical interneurons predict their physiological subtype. Neuron 48:591-604

Carney RS, Mangin JM, Hayes L, Mansfield K, Sousa VH, Fishell G, Machold RP, Ahn S, Gallo V, Corbin JG (2010) Sonic hedgehog expressing and responding cells generate neuronal diversity in the medial amygdala. Neural Dev 27:5-14. doi:10.1186/17498104-5-14

Chen L, Chatterjee M, Li JY (2010) The mouse homeobox gene Gbx2 is required for the development of cholinergic interneurons in the striatum. J Neurosci 30:14824-14834. doi:10.1523/JNEUR OSCI.3742-10.2010

Clancy B, Darlington RB, Finlay BL (2001) Translating developmental time across mammalian species. Neuroscience 105:7-17

Corbin JG, Nery S, Fishell G (2001) Telencephalic cells take a tangent: non-radial migration in the mammalian forebrain. Nat Neurosci 4(Suppl):1177-1182

De Olmos J, Alheid GF, Beltramino CA (1985) Amygdala. In: Paxinos G (ed) The Rat Nervous System, vol 1., Forebrain and MidbrainAcademic Press, Orlando, pp 223-334 
De Olmos J, Beltramino CA, Alheid GF (2004) Amygdala and extended amygdala of the rat: a cytoarchitectonical, fibroarchitectonical, and chemoarchitectonical survey. In: Paxinos G (ed) The rat nervous system, 3rd edn. Elsevier Academic Press, California, pp 509-603

Eisenstat DD, Liu JK, Mione M, Zhong W, Yu G, Anderson SA, Ghattas I, Puelles L, Rubenstein JL (1999) DLX-1, DLX-2, and DLX-5 expression define distinct stages of basal forebrain differentiation. J Comp Neurol 414:217-237

Elshatory Y, Gan L (2008) The LIM-homeobox gene Islet-1 is required for the development of restricted forebrain cholinergic neurons. J Neurosci 26;28(13):3291-3297. doi:10.1523/JNEUR OSCI.5730-07.2008

Epelbaum J, Dournaud P, Fodor M, Viollet C (1994) The neurobiology of somatostatin. Crit Rev Neurobiol 8:25-44

Ferran JL, Ayad A, Merchán P, Morales-Delgado N, Sánchez-Arrones L, Alonso A, Sandoval JE, Bardet SM, Corral-San-Miguel R, Sánchez-Guardado LO, Hidalgo-Sánchez M, Martínez-de-laTorre M, Puelles L (2015a) Exploring Brain Genoarchitecture by Single and Double Chromogenic In Situ Hybridization (ISH) and Immunohistochemistry (IHC) in Whole-Mount Embryos. In: G Hauptmann (ed) In Situ Hybridization Methods, Neuromethods. Vol 99. Springer Science+ Business Media, New York, pp 61-82. doi:10.1007/978-1-4939-2303-8_4

Ferran JL, Ayad A, Merchán P, Morales-Delgado N, Sánchez-Arrones L, Alonso A, Sandoval JE, Bardet SM, Corral-San-Miguel R, Sánchez-Guardado LO, Hidalgo-Sánchez M, Martínez-de-laTorre M, Puelles L (2015b) Exploring brain genoarchitecture by single and double chromogenic in situ hybridization (ISH) and immunohistochemistry (IHC) on cryostat, paraffin, or floating sections. In: G. Hauptmann (ed) In Situ Hybridization Methods, Neuromethods. Vol 99. Springer Science + Business Media, New York, pp 83-107. DOI 10.1007/978-1-4939-2303-8_5

Flames N, Pla R, Gelman DM, Rubenstein JL, Puelles L, Marín O (2007) Delineation of multiple subpallial progenitor domains by the combinatorial expression of transcriptional codes. J Neurosci 27:9682-9695

Flandin P, Kimura S, Rubenstein JL (2010) The progenitor zone of the ventral medial ganglionic eminence requires $\mathrm{Nk} 2-1$ to generate most of the globus pallidus but few neocortical interneurons. J Neurosci 30:2812-2823. doi:10.1523/JNEUR OSCI.4228-09.2010

Flandin P, Zhao Y, Vogt D, Jeong J, Long J, Potter G, Westphal H, Rubenstein JL (2011) Lhx6 and Lhx8 coordinately induce neuronal expression of $S h h$ that controls the generation of interneuron progenitors. Neuron 70:939-950. doi:10.1016/j.neuron.2011.04.020

Fogarty M, Grist M, Gelman D, Marín O, Pachnis V, Kessaris N (2007) Spatial genetic patterning of the embryonic neuroepithelium generates GABAergic interneuron diversity in the adult cortex. J Neurosci 27:10935-10946

Garcia-Lopez M, Abellan A, Legaz I, Rubenstein JL, Puelles L, Medina L (2008) Histogenetic compartments of the mouse centromedial and extended amygdala based on gene expression patterns during development. J Comp Neurol 506:46-74

García-Moreno F, Pedraza M, Di Giovannantonio LG, Di Salvio M, López-Mascaraque L, Simeone A, De Carlos JA (2010) A neuronal migratory pathway crossing from diencephalon to telencephalon populates amygdala nuclei. Nat Neurosci 13:680-689. doi:10.1038/nn.2556

Gelman DM, Marín O (2010) Generation of interneuron diversity in the mouse cerebral cortex. Eur J Neurosci 31:2136-2141. doi:10. 1111/j.1460-9568.2010.07267.x

Gelman DM, Martini FJ, Nóbrega-Pereira S, Pierani A, Kessaris N, Marín O (2009) The embryonic preoptic area is a novel source of cortical GABAergic interneurons. J Neurosci 29:9380-9389. doi:10.1523/JNEUROSCI.0604-09.2009
Gelman D, Griveau A, Dehorter N, Teissier A, Varela C, Pla R, Pierani A, Marín O (2011) A wide diversity of cortical GABAergic interneurons derives from the embryonic preoptic area. J Neurosci 31:16570-16580. doi:10.1523/JNEUROSCI.4068-11.2011

Gelman DM, Marín O, Rubenstein JLR (2012) The generation of cortical interneurons. In: Noebels JL, Avoli M, Rogawski MA, Olsen RW, Delgado-Escueta AV (eds) Jasper's Basic Mechanisms of the Epilepsies. 4th edn. Bethesda (MD): National Center for Biotechnology Information (US)

Ghanem N, Yu M, Long J, Hatch G, Rubenstein JL, Ekker M (2007) Distinct cis-regulatory elements from the Dlx1/Dlx2 locus mark different progenitor cell populations in the ganglionic eminences and different subtypes of adult cortical interneurons. J Neurosci 27:5012-5022

Gorski JA, Talley T, Qiu M, Puelles L, Rubenstein JL, Jones KR (2002) Cortical excitatory neurons and glia, but not GABAergic neurons, are produced in the Emx 1 -expressing lineage. J Neurosci 22:6309-6314

Götz M, Williams BP, Bolz J, Price J (1995) The specification of neuronal fate: a common precursor for neurotransmitter subtypes in the rat cerebral cortex in vitro. Eur J Neurosci 7:889-898

Gray TS, Magnuson DJ (1992) Peptide immunoreactive neurons in the amygdala and the bed nucleus of the stria terminalis project to the midbrain central gray in the rat. Peptides 13:451-460

He W, Ingraham C, Rising L, Goderie S, Temple S (2001) Multipotent stem cells from the mouse basal forebrain contribute GABAergic neurons and oligodendrocytes to the cerebral cortex during embryogenesis. J Neurosci 21:8854-8862

Hoch RV, Clarke JA, Rubenstein JL (2015) Fgf signaling controls the telencephalic distribution of Fgf-expressing progenitors generated in the rostral patterning center. Neural Dev 10(1):8 [Epub ahead of print]

Iwasato T, Datwani A, Wolf AM, Nishiyama H, Taguchi Y, Tonegawa S, Knöpfel Erzurumlu RS, Itohara S (2000) Cortexrestricted disruption of NMDAR1 impairs neuronal patterns in the barrel cortex. Nature 406:726-731

Ju G, Swanson LW (1989) Studies on the celular architecture of the bed nuclei of the stria terminalis in the rat. I. Cytoarchitecture. J Comp Neurol 280:587-602

Ju G, Swanson LW, Simerly RB (1989) Studies on the celular architecture of the bed nuclei of the stria terminalis in the rat. II. Chemoarchitecture. J Comp Neurol 280:603-621

Kumar U, Grant M (2010) Somatostatin and somatostatin receptors. In: Rehfeld, Jens F. E Bundgaard, Jens R (eds) Cellular Peptide Hormone Synthesis and Secretory Pathways. Results and Problems in Cell Differentiation. Springer Berlin Heidelberg, pp 137-184. doi:10.1007/400_2009_29

Lazzaro D, Price M, de Felice M, Di Lauro R (1991) The transcription factor $T T F-1$ is expressed at the onset of thyroid and lung morphogenesis and in restricted regions of the foetal brain. Development 113:1093-1104

Le Verche V, Kaindl AM, Verney C, Csaba Z, Peineau S, Olivier P, Adle-Biassette H, Leterrier C, Vitalis T, Renaud J, Dargent B, Gressens P, Dournaud P (2009) The somatostatin 2A receptor is enriched in migrating neurons during rat and human brain development and stimulates migration and axonal outgrowth. PLoS ONE 4(5):e5509. doi:10.1371/journal.pone.0005509

Lee S, Hjerling-Leffler J, Zagha E, Fishell G, Rudy B (2010) The largest group of superficial neocortical GABAergic interneurons expresses ionotropic serotonin receptors. J Neurosci 30:16796-16808. doi:10.1523/JNEUROSCI.1869-10.2010

Legaz I, Olmos L, Real MA, Guirado S, Dávila JC, Medina L (2005) Development of neurons and fibers containing calcium binding proteins in the pallial amygdala of mouse, with special emphasis on those of the basolateral amygdalar complex. J Comp Neurol 488:492-513 
Letinic K, Zoncu R, Rakic P (2002) Origin of GABAergic neurons in the human neocortex. Nature 417:645-649

Liodis P, Denaxa M, Grigoriou M, Akufo-Addo C, Yanagawa Y, Pachnis V (2007) Lhx6 activity is required for the normal migration and specification of cortical interneuron subtypes. J Neurosci 27:3078-3089

Ma T, Wang C, Wang L, Zhou X, Tian M, Zhang Q, Zhang Y, Li J, Liu Z, Cai Y, Liu F, You Y, Chen C, Campbell K, Song H, Ma L, Rubenstein JL, Yang Z (2013) Subcortical origins of human and monkey neocortical interneurons. Nat Neurosci 16:1588-1597. doi:10.1038/nn.3536

Marin O (2013) Cellular and molecular mechanisms controlling the migration of neocortical interneurons. Eur $\mathrm{J}$ Neurosci 38:2019-2029. doi:10.1111/ejn.12225

Marín O, Rubenstein JL (2001) A long, remarkable journey: tangential migration in the telencephalon. Nat Rev Neurosci 2:780-790

Marín O, Rubenstein JL (2003) Cell migration in the forebrain. Ann. Rev Neurosci. 26:441-483

Medina L, Abellan A (2012) Subpallial structures. In: Watson C, Paxinos G, Puelles L (eds) The mouse nervous system. Elsevier Academic Press, San Diego, pp 173-220

Medina L, Legaz I, González G, De Castro F, Rubenstein JL, Puelles L (2004) Expression of Dbx1, Neurogenin 2, Semaphorin 5A, Cadherin 8, and Emx1 distinguish ventral and lateral pallial histogenetic divisions in the developing mouse claustroamygdaloid complex. J Comp Neurol 474:504-523

Miyoshi G, Hjerling-Leffler J, Karayannis T, Sousa VH, Butt SJ, Battiste J, Johnson JE, Machold RP, Fishell G (2010) Genetic fate mapping reveals that the caudal ganglionic eminence produces a large and diverse population of superficial cortical interneurons. J Neurosci 30:1582-1594. doi:10.1523/JNEUR OSCI.4515-09.2010

Moga MM, Gray TS (1985) Evidence for corticotropin-releasing factor, neurotensin, and somatostatin in the neural pathway from the central nucleus of the amygdala to the parabrachial nucleus. J Comp Neurol 241:275-284

Morales-Delgado N, Merchan P, Bardet SM, Ferrán JL, Puelles L, Díaz C (2011) Topography of somatostatin gene expression relative to molecular progenitor domains during ontogeny of the mouse hypothalamus. Front Neuro 5:10. doi:10.3389/fnana. 2011.00010

Nery S, Fishell G, Corbin JG (2002) The caudal ganglionic eminence is a source of distinct cortical and subcortical cell populations. Nat Neurosci 5:1279-1280

Nery S, Corbin JG, Fishell G (2003) Dlx2 progenitor migration in wild type and $N k x 2.1$ mutant telencephalon. Cereb Cortex 13:895-903

Paxinos G, Franklin KBJ (2013) The Mouse Brain in Stereotaxic Coordinates. 4th Edn. Academic Press, San Diego

Pombero A, Bueno C, Saglietti L, Rodenas M, Guimera J, Bulfone A, Martinez S (2011) Pallial origin of basal forebrain cholinergic neurons in the nucleus basalis of Meynert and horizontal limb of the diagonal band nucleus. Development 138:4315-4326. doi:10. $1242 /$ dev.069534

Puelles L (2014) Development and evolution of the claustrum. In Smythies JR, Edelstein L, Ramachandran V (eds) The Claustrum. Structural, Functional, and Clinical Neuroscience. Elsevier Academic Press, San Diego, pp 119-170

Puelles L, Rubenstein JL (1993) Expression patterns of homeobox and other putative regulatory genes in the embryonic mouse forebrain suggest a neuromeric organization. Trends Neurosci $16: 472-479$

Puelles L, Kuwana E, Puelles E, Bulfone A, Shimamura K, Keleher J, Smiga $S$ et al (2000) Pallial and subpallial derivatives in the embryonic chick and mouse telencephalon, traced by the expression of the genes Dlx-2, Emx-1, Nkx-2.1, Pax-6, and Tbr-1. J Comp Neurol 424:409-438

Puelles L, Martínez S, Martinez-de-la-Torre M, Rubenstein JLR (2004) Gene maps and related histogenetic domains in the forebrain and midbrain. In: Paxinos $G$ (ed) The rat nervous system, 3rd edn. Elsevier, San Diego, pp 3-25

Puelles L, Martínez-de-la-Torre M, Bardet S, Rubenstein JLR (2012) Hypothalamus. In: Watson C, Paxinos G, Puelles L (eds) The mouse nervous system. Elsevier Academic Press, San Diego, pp 221-312

Puelles L, Harrison M, Paxinos G, Watson C (2013) A developmental ontology for the mammalian brain based on the prosomeric model. Trends Neurosci 36:570-578. doi:10.1016/j.tins.2013.06. 004

Real MA, Heredia R, del Labrador MC, Dávila JC, Guirado S (2009) Expression of somatostatin and neuropeptide $\mathrm{Y}$ in the embryonic, postnatal, and adult mouse amygdalar complex. J Comp Neurol 513:335-348. doi:10.1002/cne.21970

Remedios R, Subramanian L, Tole S (2004) LIM genes parcellate the embryonic amygdala and regulate its development. J Neurosci 24:6986-6990. doi:10.1523/JNEUROSCI.0001-04.2004

Roberts GW, Woodhams PL, Polak JM, Crow TJ (1982) Distribution of neuropeptides in the limbic system of the rat: the amygdaloid complex. Neuroscience 7:99-131

Rubenstein JL, Martinez S, Shimamura K, Puelles L (1994) The embryonic vertebrate forebrain: the prosomeric model. Science 266:578-580

Shimamura K, Hartigan DJ, Martinez S, Puelles L, Rubenstein JL (1995) Longitudinal organization of the anterior neural plate and neural tube. Development 121:3923-3933

Shimogori T, Lee DA, Miranda-Angulo A et al (2010) A genomic atlas of mouse hypothalamic development. Nat Neurosci 13:767-775. doi: $10.1038 / \mathrm{nn} .2545$

Shiosaka S (1992) Ontogeny of the central somastotatinergic system. In: Tohyama M, Björklund A, Hökfelt T (eds) Ontogeny of transmitters and peptides in the CNS. Elsevier, Amsterdam vol 10; pp 369-385

Sousa VH, Miyoshi G, Hjerling-Leffler J, Karayannis T, Fishell G (2009) Characterization of Nk6-2-derived neocortical interneuron lineages. Cereb Cortex 19(Suppl 1):i1-i10. doi:10.1093/ cercor/bhp038

Stenman J, Toresson H, Campbell K (2003) Identification of two distinct progenitor populations in the lateral ganglionic eminence: implications for striatal and olfactory bulb neurogenesis. J Neurosci 23:167-174

Stühmer T, Puelles L, Ekker M, Rubenstein JL (2002) Expression from a Dlx gene enhancer marks adult mouse cortical GABAergic neurons. Cereb Cortex 12:75-85

Sussel L, Marin O, Kimura S, Rubenstein JL (1999) Loss of Nkx2.1 homeobox gene function results in a ventral to dorsal molecular respecification within the basal telencephalon: evidence for a transformation of the pallidum into the striatum. Development 126:3359-3370

Swanson LW, Petrovich GD (1998) What is the amygdala? Trends Neurosci 21:323-331

Theiler K (1989) The house mouse. Atlas of embryonic development. 2nd edn. Springer-Verlag, New York, Berlin, Heidelberg

Valverde F, Santacana M (1994) Development and early postnatal maturation of the primary olfactory cortex. Brain Res Dev Brain Res 80:96-114

Viollet C, Lepousez G, Loudes C, Videau C, Simon A, Epelbaum J (2008) Somatostatinergic systems in brain: networks and functions. Mol Cell Endocrinol 286:75-87

Walter A, Mai JK, Lanta L, Görcs T (1991) Differential distribution of immunohistochemical markers in the bed nucleus of the stria terminalis in the human brain. J Chem Neuroanat 4:281-298 
Wang W, Lufkin T (2000) The murine Otp homeobox gene plays an essential role in the specification of neuronal cell lineages in the developing hypothalamus. Dev Biol 227:432-449

Wang W, Lo P, Frasch M, Lufkin T (2000) Hmx: an evolutionary conserved homeobox gene family expressed in the developing nervous system in mice and Drosophila. Mech Dev 99:123-137

Wang Y, Dye CA, Sohal V, Long JE, Estrada RC, Roztocil T, Lufkin T, Deisseroth K, Baraban SC, Rubenstein JL (2010) Dlx5 and Dlx6 regulate the development of parvalbumin-expressing cortical interneurons. J Neurosci 30:5334-5345. doi:10.1523/ JNEUROSCI.5963-09.2010

Watson C, Paxinos G (2010) Chemoarchitectonic atlas of the mouse brain. Academic Press, San Diego

Wonders CP, Anderson SA (2006) The origin and specification of cortical interneurons. Nat Rev Neurosci 7:687-696

Wonders CP, Taylor L, Welagen J, Mbata IC, Xiang JZ, Anderson SA (2008) A spatial bias for the origins of interneuron subgroups within the medial ganglionic eminence. Dev Biol 314:127-136

Xu Q, Cobos I, De La Cruz E, Rubenstein JL, Anderson SA (2004) Origins of cortical interneuron subtypes. J Neurosci $24: 2612-2622$
Xu Q, Tam M, Anderson SA (2008) Fate mapping Nkx2.1-lineage cells in the mouse telencephalon. J Comp Neurol 506:16-29

Xu Q, Guo L, Moore H, Waclaw RR, Campbell K, Anderson SA (2010) Sonic hedgehog signaling confers ventral telencephalic progenitors with distinct cortical interneuron fates. Neuron 65:328-340. doi:10.1016/j.neuron.2010.01.004

Yacubova E, Komuro H (2002) Stage-specific control of neuronal migration by somatostatin. Nature 415:77-81

Zaborszky L, Van den Pol A, Gyengesi E (2012) The basal forebrain cholinergic projection system in mice. In: Watson C, Paxinos G, Puelles L (eds) The Mouse Nervous System. Elsevier Academic Press, San Diego, pp 684-718

Zhao Y, Marín O, Hermesz E, Powell A, Flames N, Palkovits M, Rubenstein JL, Westphal H (2003) The LIM-homeobox gene Lhx8 is required for the development of many cholinergic neurons in the mouse forebrain. Proc Natl Acad Sci USA 100:9005-9010 